\title{
Differential Macrophage Phenotype Rewired by Hantaan Virus Constrains the Magnitude of Inflammatory Responses in Murine versus Humans
}

fanglin zhang ( $\square$ flzhang@fmmu.edu.cn )

Fourth Military Medical University

Hongwei Ma

Air Force Medical University

Yongheng Yang

Air Force Medical University

Tiejian Nie

Department of Neurosurgery, Tangdu Hospital, The Fourth Military Medical University

\section{Rong Yan}

Air Force Medical University

Yue Si

Air Force Medical University

Jing Wei

Air Force Medical University

Mengyun Li

Air Force Medical University

He Liu

Air Force Medical University

Wei Ye

Air Force Medical University

Hui Zhang

Air Force Medical University

Linfeng Cheng

Air Force Medical University

Liang Zhang

Air Force Medical University

Xin Lv

Air Force Medical University

Limin Luo

Air Force Medical University

Zhikai Xu 
Air Force Medical University

\section{Xijing Zhang}

Air Force Medical University

Yingfeng Lei

The School of Basic Medicine, The Fourth Military Medical University

\section{Article}

Keywords:

Posted Date: January 6th, 2022

DOI: https://doi.org/10.21203/rs.3.rs-1181604/v1

License: (c) (1) This work is licensed under a Creative Commons Attribution 4.0 International License. Read Full License 


\section{Abstract}

Hantaan virus (HTNV) is principally maintained and transmitted by rodents in nature, the infection of which is non-pathogenic in the field or laboratory mouse, but can cause hemorrhagic fever with renal syndrome (HFRS) in human beings, a severe systemic inflammatory disease with high mortality. It remains obscure how HTNV infection leads to disparate outcomes in distinct species. Here, we revealed a differential immune status in murine versus humans post HTNV infection, which was orchestrated by the macrophage reprogramming process and characterized by late-phase inactivation of NF-KB signaling. In HFRS patients, the immoderate and continuous activation of inflammatory monocyte/macrophage (M1) launched TNFa-centered cytokine storm and aggravated host immunopathologic injury, which can be lifethreatening; however, in field or laboratory mice, the M1 activation and TNFa release were significantly suppressed at the late infection stage of HTNV, restricting excessive inflammation and blocking viral disease process, which also protected mice from secondary LPS challenge or polymicrobial sepsis. Mechanistically, we found that murine macrophage phenotype was dynamically manipulated by HTNV via the Notch-IncRNA-p65 axis. At the early stage of HTNV infection, the intracellular domain of Notch receptor (NICD) was activated by viral nucleocapsid (NP) stimulation and potentiated the NF-KB pathway by associating with and facilitating the interaction between IKK $\beta$ and p65. At the late stage, Notch signaling launched the expression of diverse murine-specific long non-coding RNAs (IncRNAs) and attenuated M1 polarization. Among them, IncRNA 30740.1 (termed as Inc-ip65, an inhibitor of p65) bound to $\mathrm{p} 65$ and hindered its phosphorylation, exerting negative feedback on the NF-KB pathway. Genetic ablation of Inc-ip65 shifted the balance of macrophage polarization from a pro-resolution to an inflammatory phenotype, leading to superabundant production of pro-inflammatory cytokines and increasing mice susceptibility to HTNV infection or bacterial sepsis. Collectively, our findings identify an immune braking function and mechanism for murine IncRNAs in inhibiting p65-mediated M1 activation, opening a novel therapeutic avenue of controlling the magnitude of immune responses for HFRS and other inflammatory diseases.

\section{In Brief}

Ma et al. demonstrate that a unique anti-inflammatory phenotype of macrophage at the late infection phase was reprogrammed by HTNV through Notch-IncRNA-p65 pathway in mice versus humans, which might interpret the discrepant disease outcomes of natural reservoirs or HFRS patients.

\section{Highlights}

- Hyperactivation of inflammatory macrophage (M1) launches the TNFa-centered cytokine storm in human beings and correlates with disease severity of HFRS.

- The unique anti-M1 status of murine macrophage protects mice from HTNV challenge and subsequent bacterial sepsis. 
- Murine-specific Inc-ip65 downstream of the Notch pathway contributes to the late-phase inactivation of $\mathrm{M} 1$ by interacting with p 65 and inhibiting its phosphorylation.

- Lnc-ip65 deficiency aggravates systemic inflammation and sensitizes mice to HTNV infection.

\section{Introduction}

Hantaviruses are a major class of zoonotic pathogens distributed worldwide and have drawn extensive public concern with the newly reported possibility of super spread (Martínez et al., 2020). They encompass at least 58 distinct viral genotypes classified in the genus Orthohantavirus (subfamily Mammantavirinae, family Hantaviridae, order Bunyavirales), among which the Old World and New World viral lineages lead to two serious diseases in human being, namely hemorrhagic fever with renal syndrome (HFRS) prevailing in Eurasia and hantavirus pulmonary syndrome (HPS) in the Americas, respectively (Abudurexiti et al., 2019; Liu et al., 2019b). Hantaan virus (HTNV), the prototype hantavirus discovered by Lee in the 1950s, is naturally hosted by striped field mouse (Apodemus agrarius) and transmitted to human through the inhalation of contaminated aerosolized rodent secreta or excreta, causing severe HFRS in Asia with a case fatality rate as high as 15\% (Lee et al., 1981; Taylor et al., 2013). HTNV virions are enveloped and contain three negative single-stranded RNA genome segments designated as small (S), medium (M), and large (L), encoding the viral nucleocapsid protein (NP), glycoprotein precursor (GPC), and viral RNA-dependent RNA polymerase (RdRp), which collectively regulates hantaviral life cycle and determines virulence (Jiang et al., 2017). Previous studies have demonstrated that host intemperate immune responses contributed to the pathogenesis of HFRS, during which the pro-inflammatory cytokines secreted by innate immune cells upon HTNV infection, especially tumor necrosis factor a (TNFa), interleukin-6 (IL-6), and IL-8, elicited cytokine storm syndrome and were closely correlated with disease severity (Guivier et al., 2010; Khaiboullina et al., 2017; Niikura et al., 2004; Saksida et al., 2011). However, little or no tissue damage caused by aggressive inflammation could be detected in natural reservoirs or laboratory wild type murine models, conducing to the asymptomatic infection with persistent or transient HTNV carriage in rodents (Ma et al., 2017a; Schountz and Prescott, 2014; Tian et al., 2017). Less is known about what determines the magnitude of host immune response against HTNV infection.

Macrophages and their precursor monocytes belong to the host mononuclear phagocyte system (MPS), constituting the first defense line against microbial infection. Emerging evidence indicated that macrophages maintain high plasticity and heterogeneity, serving as a rheostat for immune actions (Ginhoux and Jung, 2014). Macrophages polarize into diverse functional states when encountering different microenvironments, the process of which is mediated by multiple cytokine or surveillance receptors and their downstream pathways (Ginhoux and Guilliams, 2016; Kusnadi et al., 2019; Murray et al., 2014). Stimulation by T helper type 1 (Th1) cytokines such as interferon-gamma (IFNY) or TNFa, and activation of pattern recognition receptor (PRR) such as Toll-like receptor (TLR) and RIG--like receptor (RLR), can give rise to the classical inflammatory status of macrophage (M1). M1 polarization is determined by several pivotal transcription factors, including the signal transducers and activators of 
transcription 1 (Stat1), nuclear factor-kappa B (NF-kB, especially p65/RelA), and interferon regulatory factor 5 (IRF5). Macrophages with M1 phenotype reinforce host defense by producing tremendous proinflammatory cytokines and reactive oxygen species (ROS), which have tissue-destructive properties (Murray et al., 2014; Platanitis and Decker, 2018). In contrast, Th2 cytokines (e.g., IL-4, IL-13, and IL-10) or glucocorticoids can convert macrophages to an alternative pro-resolution state (M2), which was associated with the activation of Stat3, GATA binding protein 3 (GATA3), or IRF4. Macrophages with M2 phenotype restrain host immune responses by releasing anti-inflammatory cytokines and expedite tissue repair by promoting collagen synthesis, which may retard pathogen clearance (Ginhoux and Guilliams, 2016; Kusnadi et al., 2019). The subset of monocyte has been classified as three groups, namely the classic $\left(\mathrm{CD} 14^{++} \mathrm{CD} 16^{-}\right)$, intermediate/inflammatory (M1-like, $\left.\mathrm{CD} 14^{++} \mathrm{CD} 16^{+}\right)$, and non-classic/patrolling (M2-like, $\mathrm{CD} 14^{+} \mathrm{CD} 16^{++}$) pattern, which corresponds to macrophage with resting (M0), M1 and M2 state, respectively (Guilliams et al., 2018; Italiani and Boraschi, 2014). During the anti-microbial process, monocytes are promptly mobilized and recruited into various tissues, where they differentiate into macrophages and initiate inflammatory responses; after eliminating the invading pathogens, the monocyte-derived and intrinsic tissue-resident macrophages would be reprogramed to a pro-resolution phenotype and rebuild host immune homeostasis (Guilliams et al., 2018; Merad and Martin, 2020). Dysfunction of monocyte/macrophage, or the perturbation of their functional state transition might result in uncontrolled immune responses, which contribute to the pathogenesis of multiple infectious diseases (Cole et al., 2017; Saha et al., 2016). Recent researches reported that monocyte/macrophage in human might be a determinant of hantavirus pathogenicity, for that they could not only serve viral replication and facilitate their spread, but also cause a fatal cytokine shock (Li et al., 2018; Raftery et al., 2020; Scholz et al., 2017), while the role of them in mice, particularly whether their polarization or reprogramming process benefit rodents against hantaviral infection, remains ambiguous.

Notch signaling is an evolutionarily conserved pathway in vertebrates, through which adjacent cells communicate with each other and convey genetic instructions to specify cell fates, and recent researches suggest that it may take pleiotropic actions during host innate and adaptive immune responses (Radtke et al., 2010; Shang et al., 2016). Mammal Notch ligands include Delta-like (DII1, DII3, DII4) and the Jagged (JAG1, JAG2) family members that can interact with different Notch receptors (Notch1, Notch 2, Notch 3, and Notch 4) and promote their cleavage by $y$-secretase, releasing Notch intracellular domain (NICD). NICD translocates into nucleus and associates with transcription factor complex containing CBF1/suppressor of hairless/Lag1 (CSL, also called RBP-J in mouse), converting it from repressive to active state and resulting in subsequent expression of the canonical Notch target genes, including the hairy and enhancer of split (HES) and HES-related repressor protein (HERP) transcriptional repressors (Kopan and llagan, 2009). The dual effects of Notch signaling on macrophage polarization have has been revealed with a complex but elaborate mechanism in inflammatory diseases (Foldi et al., 2016; Hu et al., 2008; Xu et al., 2012; Zhang et al., 2010). On the one hand, Notch and TLR pathways cooperated synergistically to reinforce TLR-mediated M1 activation by upregulating IRF8 synthesis and increasing the production of TNFa, IL-6, and IL-12 (Hu et al., 2008; Xu et al., 2012). On the other hand, the Notch pathway was found indispensable for a series of M2 genes expression in chitin or lymphocyte-derived DNA stimulation 
models, and the downstream gene (e.g., Hes1 and Hey1) exerted negative feedback for TLR-related M1 responses (Foldi et al., 2016; Hu et al., 2008; Zhang et al., 2010). Several studies discovered the activation of Notch pathway in monocyte or macrophage took part in the pathogenesis of acute viral infection, including dengue and influenza A virus (IAV) infection (Ito et al., 2011; Li et al., 2015), but the exact mechanisms have been largely underexplored.

Nuclear factor-kappa light chain enhancer of activated B cells (NF-KB) or Rel is a family of transcription factors that influence a broad range of physiological and pathological processes, including inflammatory or stress responses, tumorigenesis, cell proliferation, differentiation, and survival (Zhang et al., 2017). While crosstalk between Notch and NF-KB signaling in tumor cells or lymphocytes has been described during cancer progress (Ferrandino et al., 2018; Kumar et al., 2014; Maniati et al., 2011; Xiu et al., 2020), their relationship in viral diseases is unclear. Activation of canonical NF-KB/Rel by PRR pathway, in particular p65/RelA, can facilitate M1 polarization by enhancing various pro-inflammatory gene expression, which needs to be tightly regulated to prevent excessive inflammation (Platanitis and Decker, 2018; Ruland, 2011). In the resting state, p65 is bound and sequestered by the inhibitor of NF-KB (IKB) in the cytoplasm. Under infection or stress circumstances, IKB proteins will be phosphorylated by the $\mathrm{IKB}$ kinases (IKK) complex and undergo subsequent degradation, which releases p65 and potentiates its phosphorylation (Santoro et al., 2003). Phosphorylated p65 translocates into the nucleus and induces target gene expression such as TNFa, IL-6, and IL-8, and aberrant p65 activation is highly involved with the pathogenesis of cytokine storm syndrome in acute virus infection or sepsis (Rahman and McFadden, 2011; Vitiello et al., 2012). To note, the regulation process and function of NF-KB signaling seem to be controversial during hantaviral infection. Some research pointed out that HTNV triggered TLR4-dependent and p65-mediated production of inflammatory cytokines or chemokines, which was responsible for endothelium dysfunction and viral pathogenicity (Chen et al., 2017a; Yu et al., 2014; Yu et al., 2012; Zhang et al., 2014a; Zhang et al., 2015). Nevertheless, other studies suggested that HTNV NP might bind to the karyopherin importin $a$ and block the nucleus translocation of p65 induced by TNFa, hence possibly assisting virus replication by suppressing host immunity (Au et al., 2010; Taylor et al., 2009a; Taylor et al., 2009b). Elucidating the specific mechanism of NF-KB signaling in regulating macrophage reprogramming may help understand host inflammation development during HTNV infection.

Currently, numerous long non-coding RNAs (IncRNAs) have been identified to associated with proteins and act as modification switcher (e.g., Inc-DC and NKILA), location guider (e.g., lincRNA-Cox2 and THRIL), or aggregation scaffolder (e.g., NEAT1), regulating host innate immune responses at transcriptional or post-transcriptional levels (Chen et al., 2017b). Lnc-DC prevents the tyrosine phosphatase SHP1 from interacting with and dephosphorylating Stat3 by directly binding to Stat3 in the cytoplasm, controlling human dendritic cell (DC) differentiation (Wang et al., 2014). NKILA targets IKB and hinders its phosphorylation by the IKK complex, forming negative feedback loop of the NF-KB pathway in both resting and activated cells which accommodates cancer-related inflammation (Liu et al., 2015). LincRNA (long intergenic noncoding RNA)-Cox2 and THRIL are induced through the TLR1/2 pathway in macrophages, and modulates the infection-associated inflammatory responses (Carpenter et al., 2013; Li et al., 2014b). Concretely, lincRNA-Cox2 recruits the heterogeneous nuclear ribonucleoprotein (hnRNP)- 
A/B to suppress the CCL5 and Stat1 expression and enhances the occupancy of RNA polymerase II (Pol II) on the gene $/ / 6$ promoter to facilitate IL-6 production (Carpenter et al., 2013). THRIL can upregulate TNFa expression and drive inflammatory macrophage activation by guiding hnRNP-L to the genomic loci (Li et al., 2014b), which has been found to exacerbate host immune injury in sepsis recently (Chen et al., 2020a). NEAT1 collects a group of proteins, such as SFPQ and NONO, to build the subnuclear structure called paraspeckle upon stress or viral infection, which will remove the transcriptional suppression effects of SFPQ on plentiful pro-inflammatory cytokine genes or pathogen recognition receptor genes (Imamura et al., 2014; Ma et al., 2017a). Additionally, NEAT1 might also translocate to the cytoplasm where it stabilizes the mature caspase- 1 and promotes activation of inflammasomes in macrophages (Zhang et al., 2019). It is worth noting that IncRNA possesses relatively low sequence conservation across species. Several IncRNAs, such as Inc-lsm3b and Inczc3h7a, are newly found to be exclusively transcribed in mice versus human beings, which could manipulate RIG-I-mediated antiviral innate immune responses (Jiang et al., 2018; Lin et al., 2019); in contrast, another batch of immune genepriming IncRNAs (IPLs) have been identified lately in human rather than murine, which could facilitate the H3K4me3 epigenetic priming of chemokine genes and hence establish trained macrophage immunity (Fanucchi et al., 2019). Theoretically, such a phenomenon is counterintuitive as the sequence decides its biological function, but this also implies the possibility that distinguishing IncRNA transcription might be involved with the distinct immune status of different hosts against the identical pathogen infection although with unknown mechanisms.

In the present study, we documented a differential immune status determined by macrophage reprogramming after HTNV infection in murine versus human being. Human macrophages underwent perpetuated M1 activation which consolidated TNFa-centered cytokine storm in HFRS patients, whereas murine macrophages experienced the late-phase inactivation of $M 1$ polarization that curbs the augmentation of inflammation during both primary viral and secondary bacterial infection, including lipopolysaccharide (LPS)-induced or cecal slurry (CS)-caused polymicrobial sepsis. Furthermore, we demonstrated that the HTNV-activated Notch pathway could dynamically rewire murine macrophage phenotype via the Notch-IncRNA-p65 axis. NICD was activated by HTNV NP upon infection, which recruited IKK $\beta$ to the IKBa-p65 complex and reinforced the p65-mediated M1 polarization. Then, Notch signaling set off a cluster of murine-specific IncRNAs transcription, among which IncRNA 30740.1 (Incip65, an inhibitor of p65) was identified to suppress inflammatory macrophage activation. Loss- and gain-of-function assays showed that Inc-ip65 could target p65 and prohibited its phosphorylation. Together, these results demonstrate a key role for murine-specific IncRNAs in manipulating the macrophage reprogram process, which may shed light on how HTNV elicits discriminative immune responses in mouse versus human being and offer potential therapeutic strategies to alleviate HFRS and other inflammatory diseases.

\section{Results}

Hyperactivation of Inflammatory Monocyte/Macrophage Elicited by HTNV Infection Contributes to the TNFa-centered Cytokine Storm Syndrome and Endovascular Dysfunction in Human Being 
Previous studies have shown that M1-like or M2-like monocyte is the major immunological determinant for life-threatening influenza or chronic viral hepatitis, respectively (Cole et al., 2017; Saha et al., 2016), but whether monocyte activation pattern affects HFRS pathogenesis is unclear. To narrow this gap, the peripheral blood mononuclear cells (PBMC) from patients with distinct virus infection were collected, among which the monocyte subset was examined (Figure S1A) and analyzed (Figure 1A). Individuals with HTNV infection possessed a significantly increased proportion of M1-like monocytes (marked by $\mathrm{CD} 14^{++} \mathrm{CD} 16^{+}$) but a relatively reduced M2-like subset (marked by $\mathrm{CD} 14^{+} \mathrm{CD} 16^{++}$) than those with Japanese encephalitis virus (JEV), hepatitis B or C virus (HBV or HCV) infection (Figure S1A and 1A), which preliminarily implied that M1-like monocyte-mediated immune responses might be involved the pathogenicity of HTNV infection in human. HFRS is composed of five clinical stages, namely febrile, hypotensive, oliguric, diuretic, and convalescent phases (Jiang et al., 2017). To gain a comprehensive view on the role of monocyte state in the HFRS process and severity, the amount and subset of monocytes in different clinical stages were examined and analyzed. We found that the elevated monocytes (marked by CD11 ${ }^{+} \mathrm{CD} 11 \mathrm{c}^{+}$) reached the peak at the febrile or hypotensive phase and then collapsed from the hypotensive to the convalescent stage (Figure S1B and 1B), revealing that monocytes were rapidly mobilized upon HTNV infection and might make sense at the onset of HFRS. To note, although it appeared that the M1-like monocyte percentage showed no alteration in patients with varying severity across the whole clinical stages (Figure 1C), stratification analysis with disease phases showed that at the acute stage of disease, that is the febrile or hypotensive phase, the proportion of M1-like monocyte was much higher in severe/critical patients than the mild/moderate ones (Figure 1D). However, no correlation between the activation level of M2-like monocyte (marked by $\mathrm{CD} 14^{+} \mathrm{CD} 16^{++}$or $\mathrm{CD} 11 \mathrm{~b}^{+}$

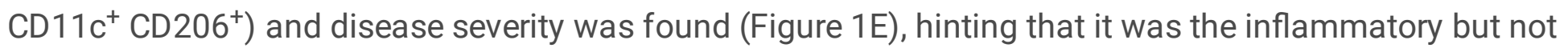
the patrolling monocytes that propelled HFRS progression.

To illustrate whether the activation of M1-like monocyte was related to host Th1 response or motivated by the viral infection, $T$ cell subsets and series of cytokines were examined at the acute phase of HFRS (Figure S1C and 1F). We found that only the percentage of regulatory $\mathrm{T}$ (Treg) cell (marked by CD $4^{+}$ $\mathrm{CD}^{2} 5^{+}$Foxp $^{+}$), but not Th1 (marked by CD $4^{+}$T-bet $^{+}$IFNY $^{+}$), Th2 (marked by CD4 ${ }^{+} \mathrm{GATA}^{+}$) or Th17 (marked by IL-17A ${ }^{+}$RORyt ${ }^{+}$), was correlated with disease severity (Figure 1F), which was consistent with previous reports (Ma et al., 2015; Saksida et al., 2011). These data indicated that the M1-like monocyte activation might be originated from HTNV infection rather than subsequent to Th1 responses. To determine the signature of inflammatory responses in HFRS, serum cytokines of 60 patients and 18 healthy controls were measured using a 40-multiplex array on a Luminex system. Totally there were 32 cytokines upregulated (Figure 1G, marked red/green/blue) and 8 cytokines remained unchanged (Figure $1 \mathrm{G}$, marked black) in HFRS patients compared with controls. Among the elevated cytokines, 20 cytokines had a statistically significant correlation with HFRS severity (Figure 1G, marked red), among which there were pro-inflammatory TNFa and IL-8, likely establishing a robust immune response and resulting in various clinical symptoms experienced by patients. The circulating concentration of BCA-1/CXCL13 and GM-CSF were negatively correlated with disease severity (Figure 1G, marked green). The left 10 upregulated cytokines, including MCP-1/CCL2 and IL-1 $\beta$ that might aggravate the patient condition in 
other acute viral diseases (Fajgenbaum and June, 2020), seemed to be unchanged in severe/critical versus mild/moderate HFRS patients (Figure 1G, marked blue). The typical Th1 cytokine IFNy that induced M1 activation, did not increase in the HFRS group compared with the healthy group, and showed no correlation with disease severity (Figure 1G, marked black), further confirming that the M1-like monocytes were not motivated by Th1 responses but possibly by HTNV infection.

The next question is that how M1-like monocytes affected disease progression upon HTNV infection. We found that M1-like monocytes were characterized with higher expression of TNFa, IL-8, and HLA-DR (Figure $1 \mathrm{H}$ ), identifying its enhanced pro-inflammatory and antigen-presenting capacity. The antiinflammatory IL-10 was also upregulated in M1-like than M2-like monocytes (Figure 1H), suggesting a compounded inflammatory response was induced by monocytes which might be involved with host immune disorder. Additionally, it was the paired production of TNFa with IL-10 in monocytes, but neither TNFa with IL-8 nor IL-8 with IL-10, that displayed a close correlation with HFRS severity (Figure S1D and 1I). To understand the timing of TNFa and IL-10 release in monocytes during the disease course of HFRS, we analyzed the clinical data of the identical patient at different disease phases and found that dynamic alteration of $\mathrm{TNFa}\left(\mathrm{CD} 11 \mathrm{~b}^{+} \mathrm{TNFa}^{+} \mathrm{IL}-10^{-}\right)$was correlated with HFRS severity (Figure $1 \mathrm{~J}$ and $\left.1 \mathrm{~K}\right)$. In patients of the mild/moderate group, TNFa production $\left(\mathrm{CD} 11 \mathrm{~b}^{+} \mathrm{TNFa}^{+} \mathrm{IL}-10^{-}\right)$reached the peak at 4 days post fever (dpf) and then presented a decreasing trend from 4dpf to 14dpf (Figure 1J, left), while IL-10 release $\left(\mathrm{CD} 11 \mathrm{~b}^{+} \mathrm{TNFa}^{-} \mathrm{IL}-10^{+}\right)$reached the peak at $7 \mathrm{dpf}$ that was later than TNFa (Figure $1 \mathrm{~J}$, middle). The mixed production of TNFa and IL-10 $\left(\mathrm{CD} 11 \mathrm{~b}^{+} \mathrm{TNFa}^{+} \mathrm{IL}-10^{+}\right)$maintained a relatively low level without evident change from $1 \mathrm{dpf}$ to $14 \mathrm{dpf}$ (Figure $1 \mathrm{~J}$, right). In patients of the severe/critical group, both TNFa and IL-10 production continuously sustained a relatively high level from $4 \mathrm{dpf}$ to $14 \mathrm{dpf}$ (Figure $1 \mathrm{~K}$ ). These data indicated that the potential mechanism of excessive inflammation in HFRS might be incriminated with the dysregulation of TNFa secretion.

The typical pathology feature of HFRS is extensively increased capillary permeability triggered by cytokine storm and hyperactivation of immune cells (Niikura et al., 2004). To clarify whether monocyte and macrophage played an indispensable role in the pathogenesis during HTNV infection, we established a cell co-culture system based on the transwell model to mirror the pathological process in vivo (Figure S2A-i), and found that HTNV could promote monocytes differentiating into macrophages (marked by

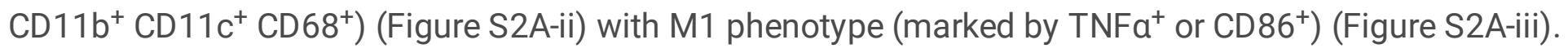
Next, we applied the adherent experiments to remove monocytes in PBMC (Figure S2B), which also blocked their differentiation to macrophages post HTNV infection. Monocyte depletion obviously improved the endovascular function after HTNV infection (Figure 1L), although the viral replication was increased in cells at the middle or bottom layer (Figure S2C). To evaluate the immune responses post HTNV infection in the co-culture system, the cytokine concentration in the upper supernatants was detected with foresaid 40-multiplex array (Figure S2D). We found that HTNV infection resulted in the upregulation of 24 cytokines (marked in red/green/blue, Figure S2D), which was similar to that in patient serum (Figure 1G), and the downregulation of 16 cytokines (marked in black, Figure S2D). Intriguingly, once the monocytes were eliminated, the production of 16 inflammatory cytokines was remarkably 
reduced, among which there were TNFa, IL-8, and IL-10 (marked in red, Figure S2D), while the production of 3 cytokines was increased, namely CCL21, IL-16 and CCL8 (marked in green, Figure S2D), with the left 21 cytokines remaining unchanged (marked in blue/black, Figure S2D). Moreover, removing monocytes also suppressed the activation of cytotoxic T lymphocytes (CTL), Th1, Th2, and Th17, which meant the monocyte was an important initiator for a series of T cell responses during HTNV infection (Figure S2E). To exclude the effects of other immune cells in PBMC, monocytes were exclusively collected through negative screening technology. To note, monocyte depletion conspicuously attenuated endothelium injury caused by HTNV (red line versus blue line, Figure 1M), and the treatment with TNFa neutralizing antibody also ameliorated the permeability alteration in the co-incubation group (brown line versus red line, Figure $1 \mathrm{M})$. Taken together, these results implied that hyperactivation of inflammatory monocyte/macrophage might contribute to the endovascular dysfunction by launching TNFa-centered cytokine storm during HTNV infection in human beings.

\section{Murine Macrophage Is Phenotypically Distinct from that of Human at Late HTNV Infection Phase}

HTNV is primarily maintained and transmitted by the striped field mice, namely Apodemus agrarius ( $A$. agrarius) widely distributed in Asia, but it causes asymptomatic infection in these natural hosts (Tian et al., 2017). Considering that undue inflammatory responses were closely associated with HFRS progression and unfavorable prognosis in human being (Figure 1G), we hypothesized this pathological process might be discrepant in rodents, which could partially decipher why hantaviruses were nonpathogenic in their reservoirs. To efficiently acquire the natural samples carrying HTNV, we first analyzed the recent prevalence of HFRS in China (Figure 2A-i), and collect the A. agrarius mice in Weihe Plain that possessed the highest incidence rate of HFRS (Figure 2A-ii). To better illuminate the natural infection process of hantaviruses in field mice, the disease phases were classified as HTNV infection negative stage (HINS), early stage (HIES), progressive stage (HIPS), and clearance stage (HICS) according to the assessment results of viral RNA and host anti-hantaviral antibody in lungs (Figure 2A-iii). Indeed, we found that $A$. agrarius mice lung tissue was more susceptible to HTNV infection rather than liver or kidney (marked by the asterisk, Figure S3A), which was consistent with previous studies (Kim et al., 2016; No et al., 2019) and insured the authenticity of our testing system. Elevated production of six inflammatory cytokines was observed in HIES than HINS, namely TNFa, IFNa, IL-1 3 , IP-10, MCP-1, and IL-10 (Figure 2B), which were also upregulated in HFRS patients (Figure 1G) and reported as pathogenic factors during hantaviral infection (Angulo et al., 2017; Chen et al., 2017a; Khaiboullina et al., 2014; Niikura et al., 2004). Of note, several pro-inflammatory cytokines, e.g., TNFa and IP-10, presented an overt declining trend from HIES to HIPS (Figure 2B) rather than continuous elevation in HFRS patients (Figure $1 \mathrm{G}$ and $1 \mathrm{~K}$ ), and the infiltrating inflammatory cells of multiple tissues did not show an obvious increase in HIES or HIPS compared with HINS (marked by the triangle, Figure S3A), indicating that mice immune system was transiently activated but timely controlled after HTNV infection.

Considering that the lethal cytokine storm was mainly initiated by monocyte/macrophage in HFRS patients (Figure S1), their activation process was specifically investigated in A. agrarius mice carrying HTNV. Murine alveolar macrophages (AMs) (marked by F4/80), which scattered in lungs from the HINS 
group, were recruited to alveolar capillaries and distributed surrounding the HTNV-infected endothelial cells (marked by CD34) in the HIES and HIPS group (Figure 2C). To evaluate the macrophage polarization state in HTNV-infected rodents, murine AMs were acquired through bronchoalveolar lavage, and the activation pattern of NF-KB and JAK/STAT pathway was detected (Figure 2D). While the total expression level of p65 increased from HINS to HIPS, its phosphorylation level reached the peak in HIES but then collapsed overtly in HIPS (Figure 2D), which was coincident with the TNFa alteration tendency in mice lungs (Figure 2B). Contemporaneously, the phosphorylation of Stat1 was kept at a high level both in HIES and HIPS, which displayed a continuous activation manner (Figure 2D). These findings implied that HTNV might dynamically manipulate murine macrophage reprogramming via NF-KB signaling in vivo.

Although M1 activation of macrophage in A. Agrarius mouse was found to be dynamically regulated during HTNV infection, it remained uncertain whether this process was beneficial or detrimental for mice. To answer this question, clodronate liposome (clophosome) was applied to eliminate monocyte and macrophage in vivo, and the pathophysiological development of HTNV infection in different laboratory murine models was evaluated. In terms of the lethal infection model of neonatal mice by HTNV (Chen et al., 2020b), clearance of monocyte and macrophage at 1dpi could significantly delay the disease onset time (Figure S3B-i), and this process could be mirrored by using TNFa neutralizing antibody (Figure S3Bii), convincing a TNFa-dependent pro-inflammatory and disadvantageous function of monocyte/macrophage at early infection phase. Interestingly, obliterating monocyte and macrophage, or application of TNFa neutralizing antibody at $5 \mathrm{dpi}$ executed no influence on mice survival situation (Figure S3B), showing that disease aggression was irreversible once cytokine storm was launched. Considering that the immune system of neonates might be immature and the fatal infection of HTNV in suckling mice was associated with their nervous system damage, adult mice were utilized to check the role of monocyte/macrophage. As for the asymptomatic infection model of adult mice by HTNV (Ma et al., 2017a), we found that depletion of monocyte and macrophage promoted the onset of disease (Figure S3C), and in the depletion group, weakened TNFa-mediated inflammation (Figure S3D-i) was accompanied by high viral loads (Figure S3D-ii). Similar results were observed in the $\mathrm{RIG}_{-\mathrm{I}^{-/}}$mice model (Figure S3C and 3D), verifying the protective role of monocyte/macrophage against HTNV infection in adult mice. In brief, monocyte/macrophage acts as a destroyer in the lethal neonatal mice model but a defender in the asymptomatic adult mice model upon hantaviral infection. The former one could partially mirror the pathogenesis of HFRS in human beings, and the latter one more possibly mimicked the natural asymptomatic infection process in A. Agrarius mice.

That being the case, we wondered why monocyte/macrophage exhibited beneficial effects in adult mice rather than human beings during HTNV infection. To tackle this issue, primary monocytes or macrophages from mice or humans were extracted and underwent HTNV infection in vitro, after which the supernatants were collected to detect the cytokine concentration at different time points (Figure 2E). Intriguingly, we found that the TNFa production from murine bone marrow-derived macrophages (mBMDM) and peritoneal macrophages ( $\mathrm{mPM} \varphi)$ increased from $0 \mathrm{hpi}$ to $24 \mathrm{hpi}$ and then descended from $24 \mathrm{hpi}$ to $48 \mathrm{hpi}$; on the contrary, the TNFa released by human monocytes (hMo) or monocyte- 
derived macrophages (hMDM) gradually upregulated from 0 hpi to $48 \mathrm{hpi}$ (upper, Figure 2E), revealing a discrepant pro-inflammatory identity of monocyte/macrophage of different species. Dissimilarly, a perpetual elevating production pattern of IFNa was found in these cell types from 0 hpi to $48 \mathrm{hpi}$ (bottom, Figure 2E), hinting that murine and human monocyte/macrophage might exhibit an analogical anti-viral function. As the outcome of host inflammation and tissue repair were mainly determined by macrophages, either originated from circulating monocytes or primary tissue-resident macrophages (Ginhoux and Jung, 2014), our following researches principally focused on the macrophages. We discovered that while total p65 expression was unremittingly enhanced in mBMDM during HTNV infection, the phosphorated p65 (S276, S468, S529, and S536) increased at first from 0 hpi to 24 hpi and then decreased from $24 \mathrm{hpi}$ to $36 \mathrm{hpi}$; however, both total and phosphorated p65 elevated in hMDM as infection prolonged (Figure 2F). In line with that, the amount of p65 in the nucleus increased from 0 hpi to $24 \mathrm{hpi}$ in both mBMDM and hMDM, but it plunged from $24 \mathrm{hpi}$ to $36 \mathrm{hpi}$ in mBMDM rather than hMDM (Figure $2 \mathrm{G}$ and $2 \mathrm{H}$ ). Some other pivotal transcription factors, which mediated M1 (such as Stat1 and IRF5) or M2 (such as IRF4) polarization, did not display a significant contrast between different species (Figure $2 \mathrm{~F}-2 \mathrm{H}$ ). Additionally, the DNA binding capacity of p65 that represented its transcriptional activity also showed a difference between mBMDM and hMDM (Figure 2I), which was in accordance with the phosphorylation and translocation alteration of p65 (Figure $2 \mathrm{~F}$ to $2 \mathrm{H}$ ). NF-KB pathway principally manipulates the M1 phenotype featured by TNFa production, which took part in host inflammation disorder during hantaviral infection (Yu et al., 2014; Yu et al., 2012; Zhang et al., 2015). Hence, possibly it was the late-phase inactivation of p65-medicated M1 response that prohibited cytokine storm in mice.

To confirm the macrophage reprogramming process by HTNV in distinct species, further experiments were performed based on murine or human macrophage cell lines. As for the murine monocyte-derived macrophage RAW264.7, murine alveolar macrophage MH-S, and human monocyte cell line THP-1-derived macrophage (PMA stimulation), they could progressively release IFNa from 0 hpi to 48 hpi (Figure S3E); nevertheless, murine RAW264.7 and MH-S cells showed a declined TNFa production pattern since $24 \mathrm{hpi}$ compared with human macrophages (Figure $2 \mathrm{~J}$ ). The dual-luciferase reporter assays suggested that the p65 activity in RAW264.7 cells reached the peak at $24 \mathrm{hpi}$ and then reduced, and to the counterpart, it maintained a continuous elevating state in THP-1-derived macrophages (Figure S4A). The p65 activity was positively correlated with HTNV dose when the multiplicity of infection (MOI) varied from 0.1 to 1 , but remained stable when $\mathrm{MOI}$ exceeded 1 (Figure S4B). To directly assess $\mathrm{p} 65$ activation status, the phosphorylation and subcellular localization of p65 were checked. We found that the phosphorylated p65 increased from 0 hpi to $24 \mathrm{hpi}$ and then decreased visibly in RAW264.7 cells, but it persistently accrued from 0 hpi to 48 hpi in THP-1-derived macrophages (Figure S4C). The macrophage cell line stabling expressing both GFP-p65 and RFP-IKBa was constructed, in which the p65 activation could be evaluated by dynamically observing the translocation of p65. Relied on the live cell imaging system, we found that p65 in the nucleus significantly reduced in RAW264.7 cells compared with that in THP-1-derived macrophages from 24 hpi to 32 hpi (Figure S4D, Video-1 for RAW264.7 cells and Video-2 for THP-1derived macrophages), revealing that the NF-KB pathway was suppressed. Finally, the NF-KB-DNA binding assays also indicated the activation of p65 collapsed since 24 hpi in RAW264 cells instead of THP-1- 
derived macrophages (Figure S4E), verifying the late-phase inactivation of p65 by HTNV in murine rather than human macrophages.

\section{Late-phase Inactivation of Inflammatory Macrophage by HTNV Confers Mice with Higher Resistance against the Secondary Endotoxin or Polymicrobial Sepsis}

It has been reported a significant association of serum endotoxin levels with hantavirus disease severity (Douglas et al., 2019), and the HFRS patients with bacterial infection had a higher risk of death (Fan et al., 2018a; Fan et al., 2018b; Yu et al., 2017), indicating that secondary LPS-induced endotoxemia or polymicrobial sepsis might be a crucial lethal factor after HTNV infection. Since HTNV reprogrammed mice inflammatory macrophage to a pro-resolution phenotype at the late infection stage (Figure 2), we wondered whether this process could prevent the augmentation of immune responses during the subsequent bacterial sepsis. To deal with this question, a sequential challenge model was established both in vitro and in vivo, and related inflammatory indicators were evaluated. The phosphorylation of p65 in mBMDM was remarkably lower post LPS stimulation in the HTNV-36 hpi group compared with the mock-infected group, while the phosphorylation of IKBa and IKKa/ $\beta$ appeared nondistinctive (Figure $3 \mathrm{~A}$ ), indicating the activation of $\mathrm{p} 65$ was regulated in itself but not its upstream factors. The LPS-induced production of pro-inflammatory cytokines (including TNFa and IL-6), chemokines (namely MCP-1), and antimicrobial ROS, but not the cytokine IL- $1 \beta$ and IL-10, was suppressed in the HTNV-36 hpi group (Figure 3B). These results suggested that the late-phase inactivation of inflammatory macrophages by HTNV could prohibit the LPS-triggered M1 polarization process, possibly through a p65-dependent manner. Oppositely, HTNV pretreatment for $12 \mathrm{~h}$ sensitized murine macrophages to a low dose of LPS stimulation, during which both phosphorylated IKBa and p65, but not IKKa/ $\beta$, increased compared with mock infection group (Figure 3C). The pro-inflammatory and anti-septic function was also enhanced with the prompt release of TNFa, IL-6, MCP-1, and ROS, but not IL-1 $\beta$ and IL-10 (Figure 3D). These findings indicated that the early-phase activation by HTNV made macrophages more easily irritated by LPS, the mechanism of which might be different from that of the late-phase reprogramming process.

Next, we want to investigate whether mice at the different HTNV infection stages exhibited discrepant immune responses against LPS-induced Gram-negative sepsis in vivo. To define the infection phase in vivo, the dynamics of HTNV NP and TNFa in various tissues were measured from 0dpi to 7dpi (Figure $3 E)$. During the whole infection process, we found that HTNV NP and TNFa maintained at a comparatively high level at 3dpi, and returned to the normal extent at 7dpi (Figure 3E), corresponding to the early- and late-infection phase, respectively. To note, the mice at the late-infection phase (7dpi) were protected from the subsequent LPS challenge with prolonged survival time (left, Figure 3F) and improved weight change (right, Figure 3F). In the mock-infected group, LPS stimulation evoked host systemic inflammatory responses, which was characterized by acute elevation of circulating TNFa and IL-6 (Figure $3 \mathrm{G}$ ), rapid hyperactivation of M1-like monocytes in peripheral blood (Figure 3H) and M1-type AMs in the lung (Figure 3I), resulting in serious tissue damage (Figure 3J); however, in the HTNV-7dpi group, the augmentation of host immune responses was effectively curbed (Figure 3G), which was possibly associated with dampened M1 polarization of monocytes and macrophages (Figure 3H and 3I), relieving 
the immunopathological injury in multiple tissues (Figure 3J). Mice of the early-phase group (HTNV-3dpi) seemed to be susceptible to lethal endotoxemia (Figure 3F), in which strengthened inflammatory monocyte and macrophage immunity (Figure $3 \mathrm{G}$ to $3 \mathrm{I}$ ) and worsened histopathological changes (Figure $3 \mathrm{~J}$ ) were found. These results intimated that HTNV infection might alter mice susceptibility to LPSinduced sepsis, and the late-phase inactivation of inflammatory monocyte and macrophages by HTNV possibly played a protective role against secondary endotoxemia in mice.

Furthermore, the CS-induced polymicrobial sepsis model was built in mice after HTNV infection or clophosome treatment, and the results evinced that both late-phase infection of HTNV and deletion of monocyte/macrophage could defend mice against lethal CS challenge (Figure S5A-i) and ameliorate their weight loss (Figure S5A-ii). Compared with the mock group, the pathological injury of lung tissues was improved in the HTNV-7dpi or clophosome group (Figure S5B), and the concentration of manifold proinflammatory cytokines in mice serum, including $T N F a, I L-6$, and IL-1 $\beta$, was distinctively downregulated in these groups (Figure S5C). No synergism of the protective effects on CS stimulation could be found when clearing monocyte/macrophage after HTNV infection (Figure S5A), and the inflammatory responses were not further refined in the double treatment group versus single management group (Figure S5B and S5C), insinuating that the beneficial influences of late HTNV infection on secondary polymicrobial sepsis were presumably depended on the monocyte/macrophage-mediated immune responses. To assess the macrophage activation pattern, the mice AMs were acquired at two days post CS challenge through bronchoalveolar lavage and related polarization genes were measured (Figure S5D and S5E). We found the pretreatment with HTNV infection (HTNV-7dpi) could suppress M1 activation by inhibiting the expression of TNFa, IL-6, IL-1 $\beta$, and Nos2 (Figure S5C), and enhancing the M2-relate genes such as Arg-1, Chil3, and Retnla (Figure S5D). These findings signified that late-phase inactivation of inflammatory macrophages by HTNV might defend rodents against lethal polymicrobial.

\section{Notch Signaling Rewires Murine Macrophage Phenotype at the Late HTNV Infection Stage}

To further dissect the murine macrophage reprogramming process, RNA sequencing (RNA-seq) of mBMDM was performed at various time points following HTNV infection $(0,12,24$ and $36 \mathrm{hr}$ post treatment). Macrophage polarization-related genes were clustered as previously reported (Murray et al., 2014), which confirmed the late-phase inactivation of inflammatory macrophages (M1) (Figure 4A, left) and the reactivation of pro-resolution phenotype (M2) (Figure 4A, right). GO terms and KEGG pathways linked to inflammatory progress and its regulation, as well as cell development and differentiation, were over-represented in the RNA-seq dataset (Figure S6A), in which multiple genes associated PRR-mediated pathway and Notch signaling changed significantly and showed a non-linear alteration pattern along with infection (Figure S6B). To decipher which factor causes late-phase inactivation of murine M1, several PRRs that has been reported as pivotal HTNV sensing receptors (Ma et al., 2017a; Yu et al., 2012; Zhang et al., 2014a) and Notch pathway components that were associated with manifold immune responses (Radtke et al., 2010; Shang et al., 2016), were interfered with separate strategies in mBMDM. Silencing TLR3 or TLR4 inhibited the TNFa production at the $24 \mathrm{hpi}$ (the early phase) but could not reverse its declining tendency from 24 hpi to 72 hpi (the late phase) (Figure 4B-i). Likewise, the late-phase 
downregulation of TNFa was also not changed in RIG-I KO or IFNAR KO mBMDM (Figure 4B-ii). In contrast, once the essential transcription factor (RBP-J) of Notch signaling was knocked out, murine macrophages (from RBP-J conditionally knockout mice, termed as RBP-J ${ }^{\text {CKO}}$ ) maintained a continuous M1 status from 24 hpi to 72 hpi (blue line vs black line, Figure 4B-iii). To investigate whether the Notch pathway played an anti-inflammatory role, the mBMDM from NICDSTOP-floxed transgenic mice was applied. Unexpectedly, igniting Notch signaling through NICD overexpression could not directly suppress TNFa release post HTNV infection; in fact, forced NICD expression even slightly promoted TNFa production (red line vs black line, Figure 4B-iii). Then there existed one possibility, that was NICD and RBP$\mathrm{J}$ might exert an inverse effect on HTNV-induced macrophage activation, of which RBP-J and downstream genes probably launched negative feedback against NICD-mediated M1 polarization. If so, blocking the downstream signaling transduction of NICD under the NICD overexpressed condition should reinforce TNFa secretion, and suppressing NICD generation might retard the early-phase activation, as well as the late-phase inactivation, of M1-type macrophage characterized by TNFa production. To verify our hypothesis, the dominant negative form of RBP-J $(\mathrm{R} 218 \mathrm{H})$ to attenuate the Notch pathway (Yin et al., 2009), and the Y-secretase inhibitor (DAPT/GSI-IX) to impede NICD generation (Xu et al., 2012) were used in the following experiments, respectively. The overexpression of $\mathrm{R} 218 \mathrm{H}$, which competitively bonded with NICD and blocked the endogenous RBP-J activation, could remarkably force M1 polarization in the NICD ${ }^{\text {STOP-floxed }}$ mBMDM by reinforcing TNFa production (Figure 4B-iii). On the other hand, the application of DAPT before infection (-24hpi) would affect TNFa release from $12 \mathrm{hpi}$ to $24 \mathrm{hpi}$ (blue line versus black line, Figure 4-iv), while its usage at the late infection stage (24hpi) subverted the collapse of TNFa production compared with the DMSO group (red line versus black line, Figure 4B-iv). These results suggested that murine Notch signaling could prompt and then put on brakes on HTNV-induced TNFa production in macrophages.

Since the Notch pathway might rewire murine macrophage phenotype, we wondered how its activation pattern was regulated by HTNV infection in detail. Consistent with the RNA-seq results (Figure S6B), the expression of various Notch receptor and ligand genes increased from $0 \mathrm{hpi}$ to $48 \mathrm{hpi}$, while that of target gene Hes 1 showed a delayed induction from 36 hpi to 48 hpi (Figure 4C-i, 4C-ii, and S7A), during which the expression of M1-related inflammatory genes showed a descending manner (Figure 4C-iii) but most of the M2-related genes exhibited an ascending pattern (Figure 4C-iv). Moreover, we found that most of the increased NICD accumulated in the cytoplasm at early-phase (0 hpi to 24 hpi, Figure 4D and S7B), whose subcellular localization was converted to the nucleus at the late-phase (24hpi to 48 hpi, Figure 4D and S7B), suggesting that HTNV could dynamically manipulate the host biological process as we previously demonstrated (Wang et al., 2019). Such activation pattern of Notch pathway was also validated in multitudinous tissues of the HTNV-infected adult mice model at multiple time points (Figure 4E and S7C), which meant that NICD stockpiled in the cytoplasm of AMs (Figure 4E), Kupffer cells (KCs), kidney or spleen macrophages (Figure S7C) at $3 \mathrm{dpi}$ and then translocated into the nucleus at $7 \mathrm{dpi}$. These data indicated that HTNV could trigger incomplete (cytoplasmic NICD production without downstream gene activation) and complete (translocation of NICD to the nucleus with downstream gene 
activation) Notch signaling in murine macrophages at the early and late infection phase, respectively, both in vitro and in vivo.

The next question is that how Notch signaling initiated by HTNV modulates the late-phase passivation of inflammatory macrophage and whether this transition matters in mice. RNA-seq results showed that the expression of most M1-related and a few M2-related genes, especially the pro-inflammatory cytokine genes such as TNFa and IL-6, were enhanced at the late infection stage in RBP-J ${ }^{C K O}$ mBMDM (Figure 4F), which was further confirmed by qRT-PCR (Figure 4G). The immunophenotype of macrophage at $36 \mathrm{hpi}$ was also subverted once RBP-J was depleted (Figure 4H-M). Increased production of TNFa, IL-6 and IL-12 at $36 \mathrm{hpi}$ indicated the reinforced pro-inflammatory function of RBP-JCKO mBMDM (Figure 4H). Upregulated expression of CD80 and CD86 of RBP-JCKO mBMDM at the late-phase showed that they harbored stronger antigen-presenting capacity (Figure 4I). Moreover, the phagocytosis ability of RBP-JCKO mBMDM was strengthened as they could phagocytose more FAM-labeled particles (Figure $4 \mathrm{~J}$ ). Although they exhibited a reduced migrating ability as shown by transwell experiments (Figure 4K), RBP-JCKO mBMDM expressed higher levels of inducible nitric oxide synthase (iNOS) and generated more ROS compared with the wild type (WT) mBMDM at $36 \mathrm{hpi}$ (Figure 4L), intimating that they could better exert anti-microbial effects, as well as trigger severer oxidative damage in situ. RBP-J ${ }^{C K O}$ mBMDM underwent a different metabolic reprogramming process, as they maintained a higher extracellular acidification rate (ECAR) (Figure 4M-i) but a lower oxygen consumption rate (OCR) (Figure 4M-ii) at $36 \mathrm{hpi}$ compared with the WT ones. This pointed out that the ablation of RBP-J forced macrophages to display a metabolic phenotype of glycolysis that highlighted the M1 polarization process (Haschemi et al., 2012), rather than the mitochondrial respiration that clued the M2 activation (Kelly and O'Neill, 2015) at the late-phase (Figure S7D). Previous research reported that the Notch pathway could strengthen the mitochondrial glucose oxidation that affected the proinflammatory macrophage activation (Xu et al., 2015). Here, we found that RBP-JCKO $\mathrm{mBMDM}$ seemed to maintain a larger amount of mitochondria but with higher damage rates (Figure 4N), which might partially interpret how the excessive oxidative stress response occurred (Figure $4 \mathrm{~L}$ ) and why the mitochondrial respiration process was blocked (Figure $4 \mathrm{M}$ ). Considering that the late-phase inactivation of murine inflammatory macrophage was involved with the quenched NFKB pathway (Figure 2F-J), precisely the reduced phosphorylation of p65 but not its upstream molecules (Figure $3 \mathrm{~A}$ and $3 \mathrm{C}$ ), we wondered that whether Notch signaling rewired murine macrophage phenotype by regulating p65 activation. To test this assumption, the mBMDM expressing GFP-p65 and RFP-IKBa were monitored with a real-time live-cell imaging system post HTNV infection. The intranuclear p65 gradually reduced from $24 \mathrm{hpi}$ to $36 \mathrm{hpi}$ in the WT mBMDM (the upper group of Figure 40, also see Video 3-6), while at the identical period, sustainable expression of p65 was detected in the nucleus in the RBP-JCKO mBMDM (the bottom group of Figure 40, also see Video 7-10). The increased phosphorylation level of p65, instead of Stat1, was found in the RBP-JCKO mBMDM at 36 hpi and 48 hpi (Figure 4P); and another key transcription factor for M1 polarization, IRF5, also slightly upregulated in the RBP-J ${ }^{\mathrm{CKO}} \mathrm{mBMDM}$ (Figure 4P). These results substantiated that the complete activation of murine Notch pathway might inhibit M1 polarization at the late infection stage by turning off the NF-KB signaling in vivo. 
To evaluate whether this process was beneficial in vivo, the neonatal and adult mice models were utilized. RBP-JCKO suckling mice showed an early onset of disease than the WT mice (Figure S7E-i), which was associated with severer inflammatory responses (Figure S7E-ii) but not with the viral load (Figure S7E-ii). Although there was no statistical difference between the survival curves of at low dosage of HTNV (Figure S7F-i), a significant collapse of survival rate was found in the RBP-JCKO group when challenged with higher viral dosages (Figure S7F-ii and -iii). More importantly, increased body weight loss of the RBP${ }^{\mathrm{CKKO}}$ adult mice from $8 \mathrm{dpi}$ to $14 \mathrm{dpi}$ was found even with low infection dose (Figure S7G-i), and murine uncontrolled TNFa responses in the RBP-JCKO group (Figure S7G-iii) were corroborated with pathological changes in spleens, which displayed as congestion and hyperplasia through gross anatomy (Figure S7Giii) and HE staining (Figure 4Q). Additionally, aggravated cell apoptosis, as well as increased intranuclear translocation of p65, was found in spleens from the RBP-JCKO mice at late-phase (Figure 4Q). The reinforced activation of signaling triggered via p65, the c-Jun N-terminal kinase (JNK), the c-Jun Nterminal kinase (ERK) or IRF5, was also found in the RBP-JCKO spleens at $7 \mathrm{dpi}$ (Figure 4R). These in vivo models suggested that the RBP-J-mediated late-phase inactivation of murine inflammatory macrophages played a protective role against HTNV infection.

Then there came another noteworthy question, namely whether such activation pattern and related regulatory model upon HTNV infection in mice also worked in human beings. In terms of the activation pattern of Notch signaling, the NICD was increasingly generated and translocated into the nucleus in hMDM from 0 hpi to 36 hpi (Figure S7H). Immunoblotting results showed that expression of Notch pathway-related receptors (Notch1 and Notch2), ligands (Jagged 1 and DII1) and target genes (Hes1) were upregulated to varying degrees from 0 hpi to 48 hpi (Figure S7I), and no accumulation of NICD in the cytoplasm was detected (Figure S7J). In addition, the mRNA transcription level of Notch signaling-related genes increased (Figure S7K), which was consistent with the immunoblotting results (Figure S7I). These results indicated that the Notch pathway was completely activated in human macrophages all through the infection stage. As for the modulatory function of Notch signaling, we found that hindering NICD generation with DAPT could significantly restrain the secretion of various pro-inflammatory cytokines at the late infection stage (48 hpi) (Figure S7L), during which the expression of manifold M1-related genes was downregulated while M2-related genes strengthened (Figure S7M). To note, DAPT could particularly constrain the phosphorylation of $\mathrm{p} 65$ rather than p-JNK or p-ERK, which would also facilitate HTNV replication from at the late infection stage (Figure S7N), suggesting that the Notch signaling might consolidate the human M1 polarization process. Furthermore, we found that the activation level of Notch signaling in monocytes was associated with disease severity (Figure S70). These data collectively demonstrated Notch signaling showed a distinct activation pattern in mice versus humans, which exerted opposite effects on macrophage reprogramming process.

\section{Murine-specific LncRNAs Downstream of the Notch Signaling Retrains M1 Polarization}

It was ambiguous that why Notch signaling regulated macrophage polarization differently in mice versus human beings. Considering that this pathway was highly conserved, we wondered whether there existed 
some other novel transcripts controlled by Notch, especially the variable IncRNAs rather than the conservative genes. The RNA-seq analysis showed that RBP-JCKO $\mathrm{mBMDM}$ harbored a wider gene density at $36 \mathrm{hpi}$ (Figure 5A-i), which was consistent with their increased expression of multiple inflammatory genes (Figure 4F), while their new transcript number was lower than that of the WT group (Figure 5A-ii). Hence, it was possible that some unknown transcripts in the WT mBMDM might hinder the inflammatory gene expression at the late infection phase compared with the RBP-J ${ }^{\mathrm{CKO}}$ group. We found that there were ninety-seven new IncRNAs were differentially expressed between the two groups (Figure 5B, Table S1 for sequence data), most of which maintained potential protein binding capacity (Table S2) according to the RBPDB database (Cook et al., 2011) and were mainly distributed on chromosome 19 (Figure 5C). To evaluate the role of newly identified IncRNAs during viral infection, their expression level was assessed at various time points. Indeed, thirty-one IncRNAs were confirmed through qRT-PCR, among which eight IncRNAs, namely 22387.1, 30740.1, 30928.1, 60100.1, 59654.1, 57001.1 and 11443.1, maintained a high endogenous transcription level and showed a fold change of more than two at the late phase (from 36 hpi to $72 \mathrm{hpi}$ ) in both HTNV and Dengue virus 2 (DENV2, which could infect mice but not induce clinical symptoms) infection group (Figure 5D and S8A). Through silence screening experiments, we found that suppressing the transcription of 22387.1, 30740.1 and 30928.1 conspicuously consolidated TNFa production in $\mathrm{mBMDM}$ (Figure 5E-i and S8B), which also could strengthen the activation of NF-KB pathway as measured by the dual-luciferase report system in RAW264.7 cells (Figure 5E-ii and S8C). These data suggested that such three IncRNAs might act as negative feedback for HTNV-induced M1 macrophage polarization.

The basic biological features of these IncRNAs were revealed. Sequence-based bioinformatic analysis (Guo et al., 2019) showed that they had low coding capability (Figure S8D), and conversation analysis based on the UCSC Genome Browser database (Haeussler et al., 2019) indicated that they were murinespecific (Figure 5F). As genetic ablation of RBP-J would largely block their transcription (Figure 5B, red labels) and there existed several RBP-J-binding DNA sequences among the upstream region of their transcription site (Figure 5G-i), the cluster of IncRNAs might be the potential downstream targets of murine Notch signaling. Restraining Notch activation via DAPT could predominantly hinder the HTNVelicited expression of those IncRNAs, and motivating Notch pathway via recombinant mouse protein of DII1 (mDII1) would drive their transcription (Figure 5G-ii). RBP-J knockout subverted the HTNV-induced expression of these IncRNAs, while replenishing RBP-J, instead of R218H, rescued this process (Figure 5G-iii). These data confirmed that the three IncRNAs were regulated by the Notch pathway. Considering that the crosstalk between TLR and Notch signaling and their synergism on inflammation had been discovered previously (Hu et al., 2008), it was assumed that TLRs might modulate the expression of the three IncRNAs indirectly. As expected, silencing TLR3 and TLR4, but not RIG-I and MDA, would inhibit these IncRNAs generation at $36 \mathrm{hpi}$ (Figure 5G-iv). Additionally, their tissue expression was evaluated through qRT-PCR, and we found that the three IncRNAs were transcribed endogenously in diversified tissues (Figure $5 \mathrm{H}$ ). The subcellular localization of them was checked by fluorescence in situ hybridization assay (FISH), and the results presented that 30740.1 and 30928.1.1 were mostly distributed in the cytoplasm, while 22387.1 was located both in the cytoplasm and nucleus (Figure 5I). As previous 
studies exhibited (Imamura et al., 2014; Wang et al., 2017; Zhang et al., 2019), IncRNA NEAT1 transcription could be induced by manifold stress (Figure $5 \mathrm{~J}$ and $5 \mathrm{~K}$ ); under the parallel circumstances, the expression of foresaid IncRNAs was enhanced with different degrees by LPS or polyIC stimulation (Figure $5 \mathrm{~J}$ ). Various RNA viruses, such as Sendai virus (SeV), vesicular stomatitis virus (VSV) and enterovirus 71(EV71), propelled the expression of these IncRNAs at the late infection phase (from $48 \mathrm{hpi}$ to $72 \mathrm{hpi}$ ) (Figure 5K-i and S8E), while it seemed that DNA viruses, such as herpes simplex virus type 2 (HSV-2), could not activate their transcription all through the whole infection stages (Figure $5 \mathrm{~K}$-ii). This indicated that these murine-specific IncRNAs might play an important regulatory role against M1 polarization during RNA virus infection process. Additionally, the of expression these IncRNAs was correlated with HTNV MOIs as shown by qRT-PCR (Figure 5L-i) and Northern blot (Figure 5L-ii), suggesting that they might be directly modulated by viruses rather than cytokines.

To fully investigate the role of these Notch-downstream IncRNAs on macrophage polarization, the locked nucleic acids (LNAs) were applied to intervene their expression in NICD ${ }^{\text {STOP-floxed }}$ mBMDM (Figure S9A), which might achieve better inhibitive effects than siRNAs (Figure S8B and S8C). We found that silencing $22387.1,30740.1$ or 30928.1 could significantly hinder the macrophage phenotype transition from $\mathrm{TNFa}^{+}$ IL-10- $10^{-} \mathrm{TNFa}^{+} \mathrm{IL}-10^{+}$or $\mathrm{TNFa}^{-} \mathrm{IL}-10^{+}$at the late infection phase (24 hpi to $36 \mathrm{hpi}$ ) (Figure $5 \mathrm{M}-\mathrm{i}$ and $5 \mathrm{M}-$ ii). For the HTNV-infected macrophages $\left(\mathrm{NP}^{+}\right)$, they tended to display an anti-inflammatory $\mathrm{M} 2$ phenotype (mainly in group a featured by $\mathrm{TNFa}^{-} \mathrm{IL}-10^{+}$and group b characterized with $\mathrm{TNFa}^{+} \mathrm{IL}-10^{+}$) (Figure $5 \mathrm{M}-\mathrm{i}$ and $5 \mathrm{M}$-iii), while in terms of the bystander macrophages $\left(\mathrm{NP}^{-}\right)$, they tended to display a proinflammatory and anti-microbial (iNOS ${ }^{+}$) M1 phenotype (mainly in group c featured by $\mathrm{TNFa}^{+} \mathrm{IL}_{-}-10^{-}$and group b characterized with $\mathrm{TNFa}^{+} \mathrm{IL}-10^{+}$) (Figure $5 \mathrm{M}-\mathrm{i}$ and $5 \mathrm{M}$-iii). Intriguingly, knocking down those IncRNAs could remarkably consolidate the anti-microbial function of macrophages and suppress HTNV replication from $24 \mathrm{hpi}$ to $36 \mathrm{hpi} \mathrm{(Figure} \mathrm{5M-iii} \mathrm{and} \mathrm{5M-iv).} \mathrm{At} \mathrm{the} \mathrm{late} \mathrm{infection} \mathrm{phase} \mathrm{(36} \mathrm{hpi),} \mathrm{interfering}$ $22387.1,30740.1$ or 30928.1 improved the pro-inflammatory capacity by motivating $\mathrm{CCR} 7^{+} \mathrm{IL}-6^{+}$ macrophages (Figure $5 \mathrm{~N}$ ), which were accompanied by the reduction of chemokine receptor expression $\left(\mathrm{CCR} 2^{+} \mathrm{CX} 3 \mathrm{CR} 1^{+}\right)$(Figure 50) and impaired chemotaxis ability (Figure 5P). To note, once these IncRNAs were knocked down, both the phagocytosis and antigen-presenting functions were improved (Figure 5Q and 5R), while the expression of CD206 (M2 marker) was dramatically decreased (Figure 5S). The macrophage metabolic process was also converted to M1-related glycolysis type in the LNAs interfering group (Figure T). In the WT mBMDM, similar results were discovered, which meant silencing 22387.1, 30740.1 or 30928.1 could reinforce TNFa and IFNa production at $36 \mathrm{hpi} \mathrm{(Figure} \mathrm{S9B-i} \mathrm{and} \mathrm{S9B-ii),} \mathrm{but}$ restrain IL-10 and generation (Figure S9B-iii). Loss function of those IncRNAs also suppressed HTNV replication by blocking NP expression (Figure S9C), enhanced M1-related but hindered M2-related gene expression (Figure S9D). Furthermore, compensating these IncRNAs might partially offset the pro-M1 effects in RBP-JCKO mBMDM at 36 hpi by controlling the percentage of $\mathrm{TNFa}^{+}$macrophages (Figure S9E and $5 \mathrm{U}$ ), verifying the negative feedback launched by these IncRNAs. In Brief, the cluster of RBP-Jtargeted IncRNAs facilitated macrophage transformation from pro-inflammatory to pro-resolutory phenotype at the late HTNV infection phase. 


\section{Lnc-ip65 Obstructs M1 Polarization by Interacting with and Inhibiting P65 Phosphorylation}

Albeit a series of murine IncRNAs have been identified as negative regulators of M1 activation post HTNV infection (Figure 5), the specific modulatory mechanisms were obscure. Considering that the expression of IncRNA 22387.1, 30740.1 and 30928.1 was attenuated at the early infection stage (from 0 hpi to 24 hpi) (Figure 5D and S8A), overexpression experiments were applied and then M1-related signaling was assessed at 24hpi (Figure 6A). Reinforced expression of these IncRNAs respectively or simultaneously could repress p65 and Stat1 phosphorylation compared with the vector control group (Figure 6A). As the transcription of such IncRNAs was induced at the late infection stage (from $36 \mathrm{hpi}$ to $72 \mathrm{hpi}$ ) (Figure 5D and S8A), knockdown experiments were performed and we found that both p65 and Stat1 phosphorylation levels were augmented compared with the negative control (NC) group under IncRNA silencing circumstances (Figure 6A). To note, intervening IncRNA expression would not affect the phosphorylation of IKKa/ $\beta$ and IKBa (Figure $6 \mathrm{~A}$ ). The IncRNA-protein interaction propensity was computed with catRAPID omics (Armaos et al., 2021), and the results predicted that 30740.7 might bind to pivotal transcription factors of NF-KB or STAT family (Figure 6B-i), and the RNA-binding protein immunoprecipitation (RIP) experiments further confirmed the interaction between 30740.7 and p65 with either overexpression (Figure 6B-ii) or natural infection system (Figure 6B-iii). Here, considering that murine IncRNA 30740.1 showed better response against different RNA virus infection than 22387.1 or 30928.1 (Figure 5K and S8E), we mainly focused on the function of 30740.1, which was termed as the inhibitor of p65 (Inc-ip65). Lnc-ip65 colocalized with p65 at the resting status or late infection stage in mBMDM during natural infection process as shown by RNAScope, at the time points of which fewer nucleus p $65^{+}$cells could be detected compare with 24 hpi (Figure 6C), indicating Inc-ip65 possibly bond to p65 and restricted its translocation into the nucleus. Their interaction was also found post DENV infection or polyIC/LPS stimulation in the overexpression system of RAW264.7 as shown by FISH (Figure 6D). Additionally, Inc-ip65 ${ }^{-/-}$RAW264.7 showed a sustained p65 activation from 24 hpi to $36 \mathrm{hpi}$, whose subcellular localization in the nucleus was limited at the late infection stage in the WT cells (Figure 6E, also see Video-11 for WT and Video-12 for Inc-ip65 ${ }^{-/-}$RAW264.7). Knocking out Inc-ip65 would reverse the declining p65 phosphorylation, principally at Ser 276, Ser 529 and Ser 536 (but not Ser 468) (Figure $6 \mathrm{~F}$ ), which was consistent with the knockdown experiments (Figure 6A).

To investigate the exact interaction region of p65 with Inc-ip65, different mutants of p65 were constructed according to the potential RNA-binding domain (Figure 6G-i). The 1-549, 1-300, 401-549 and 401-500 amino acids (aa) segments of p65, but not 1-260 and 301-400 aa, could bind to Inc-ip65 as measured by RIP (Figure 6G-ii), and the interaction relationship was further verified through RNAScope experiments (Figure 6G-iii). The results suggested that Inc-ip65 possibly was absorbed to the region adjacent to phosphorylation points (S276, S529 and S536), which would interfere with their phosphorylated process through conformational hindrance. To validate whether this steric effect matters, competitive experiments were implemented through exogenously expressing p65 (401-500 aa). As expected, p65 (401-500 aa) could recruit and remove the negative effects of Inc-ip65, strengthening the endogenous p65 phosphorylation (Figure 6I-i) and its translocation into the nucleus (Figure 6I-ii and 6I-iii). 
To unearth the functional region of Inc-ip65, the secondary structure and relative thermodynamic free energy were analyzed with RNAfold (Mathews et al., 2004), and different truncated segments were designed and constructed based on the structure stability (Figure $6 \mathrm{~J}$ ). We found that the middle part of Inc-ip65 (1001-2000 nucleotides/nt, including 1001-1500 and 1501-2000 nt) could notably hinder p65 phosphorylation at S529 and S536, and the head part of Inc-ip65 (1-1000 nt, including 1-500 and 501$1000 \mathrm{nt}$ ) seemed to maintain better inhibitory effects on the S276 phosphorylation, both of which (head and middle part of Inc-ip65) could not affect the T254 and S311 phosphorylation of p65 and the activation of IKBa at $24 \mathrm{hpi}$ (Figure 6K). The tail region of Inc-ip65 (2001-3514 nt, including 2001-3000 and 3001-3514 nt) could not influence p65 phosphorylation or IKBa activation (Figure 6K). Likewise, exogenous expression of the head or middle region of Inc-ip65 would weaken TNFa but strengthen IL-10 mRNA transcription (Figure 6L). RNAScope showed that it was the head or middle region of Inc-ip65 that interacted with p65 and restrained its translocation into the nucleus in HTNV-infection macrophages (Figure 6M). Moreover, RIP results manifested that p65 (1-300 aa) and p65 (401-500 aa) bond to the head and middle part of Inc-ip65, respectively (Figure $6 \mathrm{~N}$ ), proving the hypothesis that Inc-ip65 was attached to the serine nearby area and exerted steric effects.

\section{Lnc-ip65 Deficiency Aggravates Systemic Inflammation and Sensitizes Mice to HTNV Infection}

To further elucidate the physiologically protective role of Inc-ip65 in anti-inflammatory innate immunity against HTNV infection, Inc-ip65 de匹cient mice (Inc-ip65 ${ }^{-/-}$) were generated using CRISPR/Cas9 technology (Figure S10A-i) and their deficient efficiency was verified (Figure S10A-ii to S10A-iv). There were no evident physiological or behavioral differences of normal body size and weight for neonatal or adult Inc-ip65 $5^{-/-}$mice compared with their wild-type (WT) littermates Inc-ip65 ${ }^{+/+}$, while the transgenic mice showed a shortened lifespan (Figure S10B). As for the neonatal mice model, the disease course in Inc-ip $65^{-/-}$group post HTNV infection was characterized with early onset and prompt death (Figure $\mathrm{S} 10 \mathrm{C}$ ). As for the adult mice model, we found that Inc-ip65 delcient mice were more susceptible to HTNV infection as they showed a greater lethality (red line versus black line, Figure 7A) and severer weight loss (red line versus black line, Figure 7B) than WT mice when given a high challenge dose of HTNV. This pathogenesis process could be partially rescued through anti-TNFa antibody treatment (blue line versus green line, Figure 7A and 7B). Continuously higher concentration of serum TNFa and IL-6 at the early infection course, as well as lower IL-10, was detected in Inc-ip65 $5^{-/-}$mice rather than the WT ones (Figure 7C), suggesting that excessive inflammatory response might be the primary cause for HTNV-triggered host death in Inc-ip $65^{-/-}$mice.

Then the host systemic inflammatory injuries post HTNV infection, specifically at the late infection stage, were evaluated. The pro-inflammatory cytokine production was significantly consolidated in the Incip $65^{-/-}$lung tissue from $4 \mathrm{dpi}$ to $6 \mathrm{dpi}$, in which the IL-10 expression was decreased (Figure 7D). Consistently, massive immunocyte in ltration and interstitial exudation were revealed in the Inc-ip $65^{-/-}$ lung tissue (Figure 7E-i), which was accompanied by the deteriorated apoptosis process (Figure 7E-ii). Murine AMs $\left(\mathrm{F} 4 / 80^{+}\right)$, alveolar epithelial and stromal cells from the Inc-ip65 $5^{-/}$group showed higher NICD 
production and iNOS expression (Figure 7E-iii), in which the phosphorylation levels of p65 and Stat1 were also remarkably strengthened (Figure 7E-iv), uncovering that there might exist uncontrolled inflammatory macrophage activation in HTNV-infected Inc-ip $65^{-/-}$mice. Furthermore, the activation of M1-related transcription factors was evaluated, and we found that the phosphorylation of p65 and Stat 1 were reinforced in Inc-ip65 deficient AMs (Figure 7F). Intriguingly, though the AMs of Inc-ip65 ${ }^{-/-}$mice displayed enhanced inflammatory and anti-viral phenotype (Figure 7F), the HTNV replication was not limited, especially in alveolar epithelial and interstitial cells (Figure 7D and 7E-v), suggesting Inc-ip65 might influence other biological functions in non-immunocytes. Serious inflammatory responses were examined in the Inc-ip65 $5^{-/-}$mice liver tissue, in which the HTNV replication was restrained (Figure 7G). Morphologically, HTNV infection triggered more inflammatory cell infiltration among the hepatic lobule and induce hepatocyte pyknosis and apoptosis (Figure $7 \mathrm{H}-\mathrm{i}$ and $7 \mathrm{H}$-ii). Excessive inflammatory activation $\mathrm{KCs}$ were found in Inc-ip $65^{-/-}$mice and featured by higher iNOS production and p65/Stat1 phosphorylation (Figure $7 \mathrm{H}$-iii and $7 \mathrm{H}$-iv), which was accompanied by reduced viral replication (Figure $7 \mathrm{H}-$ v). Similar to the Inc-ip65-/- AMs, the Inc-ip65 deficient KCs showed an upregulated phosphorylation level of p65 at $6 \mathrm{dpi}$, while differently, the generation of phosphorated Stat1 seemed to be affected (Figure 7l). In the Inc-ip $65^{-/-}$spleens, the pro-inflammatory cytokine production was slightly increased at $6 \mathrm{dpi}$ (Figure 7J), while the pathological section indicated a prominent white pulp reduction and tissue apoptosis (Figure 7K-i and 7K-ii). Likewise, knocking out Inc-ip65 would force M1 macrophage polarization (marked by iNOS and IL-12, as well as p-p65 and p-Stat1) and restrict viral replication in murine spleens at the late HTNV infection stage, in which the M2 macrophage activation (marked by CD206, as well as IL-10) in spleens was largely blocked (Figure 7K-iii to 7K iv, and 7L). Augmented inflammatory responses were found in murine kidneys (Figure S10D and S10E), while the alteration in hearts (Figure S10F and S10G) or brains (Figure S10H and S10I) seemed to be insubstantial (Figure S10F to S10I). The overall inflammation score evaluation in various organs suggested that there existed more serious immunopathological alteration for the lung, liver and spleen in Inc-ip65 $5^{-/-}$mice compared with WT ones at $6 \mathrm{dpi}$ (Figure 7M). Additionally, murine heat and mechanical hypersensitivity were measured at different time points post HTNV challenge, and we found the responsive latency or threshold was decreased in the Inc-ip 65 $5^{-/-}$mice (Figure 7N), which hinted that Inc-ip65 knockout might aggravate host inflammation. Finally, the classical sepsis models were brought in, and we found that Inc-ip $65^{-/-}$mice were susceptible to LPS or CS challenge (Figure $7 \mathrm{~N}$ ), which indicated deteriorated inflammation occurred in the Inc-ip $65^{-/-}$mice. Taken together, the in vivo data pointed out the Inc-ip65 played a critically protective role in maintaining host immune homeostasis post HTNV infection.

\section{NICD Is Activated by HTNV NP and Facilitates NF-KB Signaling in Macrophages at the Early Stage}

Since murine Notch signaling was initially activated upon HTNV infection in both murine (Figure S7A and S7B) and human (Figure S7I and S7J) macrophages, we were curious about the role of NICD itself during the HTNV-induced macrophage polarization process. Previous studies have shown complicated crosstalk between the Notch and NF-kB pathway (Szklarczyk et al., 2021) (Figure 8A), and here we found that NICD directly bond to not only p65 but also IKK 3 (Figure 8B), which could be detected during the HTNV 
infection process (Figure 8C). To determine whether NICD participated in HTNV-triggered activation of NFKB pathway at the early infection phase, NICD was exogenously expressed in RBP-JCKO $\mathrm{mBMDM}$, in which the negative regulation caused but Notch downstream IncRNAs was blocked. NICD promoted the phosphorylation and degradation of IKBa even at low challenge dose of HTNV, which facilitated p65 activation from $12 \mathrm{hpi}$ to $24 \mathrm{hpi}$, but it could not affect the generation of phosphorylated IKK $\beta$ (Figure 8Di). The DNA-binding activity of NF-KB was enhanced by NICD (Figure S11A-i), as well as the production of TNFa (Figure S11A-ii). Alternatively, suppressing NICD production with DAPT would considerably weaken p65 phosphorylation but strengthened Stat1 activation even at high challenge dose of HTNV, (Figure 8Dii), in which the NF-KB activity and TNFa expression were also downregulated (Figure S11B). These data suggested that NICD might act as a ferry role to accelerate the interaction between IKK $\beta$ and p65, which could efficiently drive NF-KB signaling by propelling IKBa degradation and p65 phosphorylation.

Different protein mutants of NICD, p65 or IKK $\beta$ were constructed based on their intrinsic domains and used in co-immunoprecipitation experiments to uncover the specific interaction region (Figure 8E). In terms of the combination between NICD and p65, we found that NICD could pull down the p65 (1-300 aa) and p65 (401-549 aa) (Figure 8F-i), and the later ones also precipitated with NICD (Figure 8F-ii). Truncated NICD segments containing ankyrin (ANK) repeat domain, namely NICD-ANK, NICD- $\triangle$ RAM and NICD$\triangle \mathrm{PEST}$, were enriched by p65 (Figure 8G-i); and likewise, these truncated sections also could recruit p65 (Figure 8G-ii). As for the interaction of NICD with IKK $\beta$, we found that NICD pulled down the IKK $\beta$ mutants containing serine/threonine protein kinases catalytic (STKc) domain (Figure 8H-i), namely IKK $\beta$-STKc, IKK $\beta-\triangle N E M O$, which in turn immunoprecipitated with NICD (Figure 8H-ii). On the other hand, it was the NICD mutant including RAM or ANK that collaborated with IKKß (Figure 8I). Considering that Inc-ip65 could bind to the p65 (1-300 aa) and p65 (401-549 aa) (Figure 6G), the identical region that mediated the combination between p65 and NICD (Figure 8F), we wondered whether Inc-ip65 negatively influenced the NICD-p65 interaction. In fact, Inc-ip65 (full length), as well as Inc-ip65 (1-1000 nt) and Inc-ip65 (1001$2000 \mathrm{nt}$ ) that were enriched by p65 (1-300 aa) and p65 (401-500 aa), respectively (Figure 6N), could significantly restrain the NICD-p65, but not NICD- IKK 3 interaction (Figure 8J). This indicated Inc-ip65 might form negative feedback for NICD-mediated p65 activation. Interestingly, we also found that HTNVinduced Notch signaling was also crucial for early-phase activation of inflammatory macrophage in human beings, as intervening NICD generation would synchronously affect p65 phosphorylation (Figure $8 \mathrm{~K}$ ), which was consistent with the results in murine cells (Figure 8D). Replenishing murine-specific Incip65 in hMDM could conspicuously prohibit p65 and Stat1 phosphorylation, and consolidate the activation of Stat3 and IRF4 that mediated the M2 polarization process (Figure 8L). The release of the pro-inflammatory cytokine, especially TNFa, IL-6 and IL-8, was prominently decreased in human macrophages once Inc-ip65 was exogenously expressed, in which the IL-10 production was enhanced but the IFNa generation remained unchanged (Figure $8 \mathrm{M}$ and $8 \mathrm{~N}$ ). These results indicated that compensating Inc-ip65, which possibly rewired the macrophage phenotype from $M 1$ to $M 2$, might be a potential antiinflammatory therapeutic strategy in HFRS patients. 
Another important question is that how HTNV infection activated Notch signaling. Bioinformatic analysis by P-HIPSTer (Lasso et al., 2019) indicated that the viral proteins, including L protein, G1/G2 GP and NP of HTNV, might not interact with the Notch components (Figure 80). Nevertheless, we found that it was NP stimulation, but not the exogenous expression of HTNV RNA segments or treatment with virus-like particles (VLP) that were composed with HTNV G1/G2 GP as we previously constructed (Cheng et al., 2016; Ma et al., 2017a), that promoted NICD production at $24 \mathrm{hpi}$ (Figure 8P-i), after which the mRNA transcription level of TNFa and Hes1 was upregulated at $36 \mathrm{hpi}$ (Figure 8P-ii and 8P-iii), indicating that NP might activate Notch signaling. DAPT inhibited the NP-induced inflammatory gene expression in mBMDM (Figure 8P-iv and 8Q), suggesting that NP might trigger M1 activation via Notch pathway. As no similar domains were found between HTNV NP and Notch ligands (Figure S11C), NP might indirectly propel Notch signaling, possibly through TLR pathway as previously reported (Hu et al., 2008). To evaluate the relationship between NP and host pathogenesis, the serum NP were detected from HFRS patients, and the results showed that the NP production was positively associated with disease severity (Figure S11D) and the percentage of M1-like monocytes (Figure S11E), suggesting HTNV NP might arouse immune imbalance and contribute to HFRS pathogenesis. A series of non-neutralizing antibodies against HTNV-NP has been screened as we previously reported (Xu et al., 2002), and we found 1A8 could efficiently reverse NP-mediated M1 activation by restraining the TNFa and iNOS production (Figure 8Q). To ensure the functional epitope, different truncated NP proteins were applied, and the 0.3NP (containing 100 aa translated from the 1-300 nt of S segment) could mimic the pro-M1 effects of 1.3NP (full length) which process could be blocked by 1 A8 or DAPT (Figure 8R and S11F). To note, 1A8 treatment improved the survival curve of the lethal neonatal mice model (Figure $8 \mathrm{~S}$ ). For $1 \mathrm{~A} 8$ treated mice, the activation of

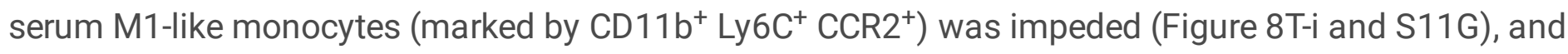
fewer inflammatory macrophages, marked by CD11 $\mathrm{b}^{+} \mathrm{Ly}_{6 \mathrm{C}^{+}}$(Figure 8T-ii and $\mathrm{S} 11 \mathrm{H}$ ) or $\mathrm{F} 4 / 80^{+} \mathrm{CD} 11 \mathrm{C}^{+}$ iNOS $^{+} \mathrm{CD}^{206}{ }^{-}$(Figure 8T-iii and S11I), were found, suggesting that $1 \mathrm{~A} 8$ retarded host inflammatory responses. The early application of neutralizing antibody 3D8 (1 dpi) could protect neonatal mice from lethal HTNV challenge as we previously reported, while the beneficial effects would disappear if 3D8 was used later than $5 \mathrm{dpi}$ (red line versus black line, Figure $8 \mathrm{U}$ ). It was noteworthy that combined application of 1A8 with 3D8 at 5dpi could regain the protective effects (green line versus blue line, Figure 8U), suggesting that inhibition of excessive inflammation might spare more time for the host to restrain and eliminate HTNV. In brief, NP itself might promote Notch signaling and evoke M1 activation during HTNV infection, which could be blocked by anti-NP antibody $1 \mathrm{~A} 8$ both in vitro and in vivo.

\section{Discussion}

NF-kB is provoked under multiple stress circumstances, especially upon acute viral and bacterial infection, and constitutively active in various tumors, acting as a pivotal factor in determining cell fate and host disease outcome (Santoro et al., 2003; Zhang et al., 2017). Numerous negative modulators of the NF-KB pathway, such as deubiquitinase TNFAIP3/A20 (Priem et al., 2020), ubiquitin ligase SOCS-1 (LV et al., 2020), a group of miRNAs (Boldin and Baltimore, 2012) and a few IncRNAs (Liu et al., 2015; Shang et al., 2019), etc. have been identified as potent anti-inflammatory molecules. However, it is unknown that

Page 24/83 
whether there existed distinctive regulatory mechanisms for NF-kB signaling between different species. In this study, we reported that several murine-specific IncRNAs controlled by the Notch pathway, particularly Inc-ip65, formed the negative feedback loop to prohibit sustained or excessive activation of NF-kB pathway in macrophages. This partially deciphers how hantaviruses triggered a divergent immune status in mice versus humans, and interprets a novel mechanism about why hantaviruses are nonpathogenic to the adult rodents but contribute to HFRS in human beings.

Hantaviruses have drawn worldwide attention as emerging zoonotic viruses. Though it was universally acknowledged that the pathogenesis of HFRS or HPS caused by hantaviruses was highly involved with immoderate immune responses (Brocato and Hooper, 2019; Vaheri et al., 2013), the key regulator that governs the initiation and conversion of host inflammation still remains unclear. Preceding researchers have observed that massive NK cell expansion and activation (Braun et al., 2014), as well as uncontrolled virus-specific T cell responses (Terajima and Ennis, 2011), in hantavirus disease progress, which might directly execute tissue-destructive effects but not manipulate the inflammatory status. Meanwhile, the relationship between Treg cells and hantaviral immunopathogenesis was still under debate (Koivula et al., 2014; Li and Klein, 2012). Herein, we found that it was the activated inflammatory monocytes or macrophages, but not T cell subsets, that showed a correlation with the HFRS disease severity, and proved that their hyperactivation would trigger TNF-a centered cytokine storm and lead to the turbulence of $T$ cell response. Another intriguing question is that why hantaviruses would not cause lethal infection in rodent reservoirs (Easterbrook and Klein, 2008; Schönrich et al., 2008; Schountz and Prescott, 2014). Previous studies have shown that hantavirus might interrupt host IFN production by various strategies (Hannah et al., 2008; Vera-Otarola et al., 2020), resist TRAIL-medicated cell death (Solà-Riera et al., 2019), disturb virus-specific CTL-associated pathogen clearance (Gupta et al., 2013) and promote the Tregassociated immune suppression (Easterbrook et al., 2007; Schountz et al., 2007), thus resulting in viral persistence in rodents. Little is known about why hantavirus-infected mice were prevented from excessive inflammation. Hantaviral NP was produced in abundance in infected cells, principally host vascular endothelial cells, which might competitively bind to the karyopherin and impede the nucleus translocation of p65 induced by TNFa (Taylor et al., 2009a). Conversely, we found that HTNV NP did not increase significantly from 24hpi to $36 \mathrm{hpi}$ in murine or human macrophages (Figure $2 \mathrm{~F}$ to $2 \mathrm{H}$ ), implying that HTNV might cause abortive infection in immune cells, and the reprogramming process featured by p65 inactivation might be caused by other factors. We identified the differential macrophage phenotype rewired by HTNV, which was consistent with prevenient studies (Au et al., 2010; Plekhova et al., 2005), and further demonstrated the Notch-IncRNA-p65 pathway constrains the magnitude of inflammatory responses in murine versus humans, adding novel insights into the immunological mechanisms and identify new possible targets for intervention.

The Notch pathway controls the embryonic development, cell differentiation and tissue homeostasis in multiple organs, mainly by inhibiting specific signals required for cell-type specification, whose dysregulation is highly associated with several human disorders, including cancer and hereditary diseases (Chabriat et al., 2009; Ferrandino et al., 2018; Kopan and llagan, 2009). Recent evidence suggests that Notch signal is an important modulator of macrophage-mediated immune responses (Foldi 
et al., 2016; Xu et al., 2012; Xu et al., 2015), while the downstream molecular mechanisms, peculiarly during acute viral infection, largely remain elusive. JEV induces the expression of miRNA let-7a/b, which will activate the Notch-TLR7 pathway and enhance microglia-medicated neuroinflammation (Mukherjee et al., 2019). DENV upregulates the expression of Notch ligands through IFN signaling in monocytes and macrophages, which would further modulate the host Th1/Th2 differentiation during adaptive immune response but not affect viral replication (Li et al., 2015). IAV challenge elicited the Notch ligand DIl1 expression on macrophages through RIG-I but not TLR3-TRIF pathway, the blockage of which with GSI would result in higher mortality caused by excessive inflammation and impaired production of IFN-y in lungs post IAV infection (Ito et al., 2011). These data showed that Notch signaling might exert either pro- or anti-inflammatory effects by rewiring macrophage during viral diseases, while it was opaque that how Notch played a dual role and which factor determined the ultimate denouement. We reported that the murine Notch pathway was dynamically activated by HTNV, which would rewire the macrophage phenotype at different infection phases. At the early infection stage, NICD accumulated in the cytoplasm and facilitate p 65 phosphorylation by interacting with both p 65 and IKK $\beta$, thus promoting M1 polarization. At the late infection stage, NICD translocated into the nucleus and motivated various murinespecific IncRNAs, among which the Inc-ip65 would bind to and suppress p65 phosphorylation, reprogramming macrophages from $\mathrm{M} 1$ to anti-M1 state.

Cytoplasmic IncRNAs have previously been reported as vital immune regulators by affecting mRNA stability and translation, or influencing protein function (Statello et al., 2021; Zhang and Cao, 2021). LncRNA Sros1 stabilized the Stat1 mRNA in macrophage by blocking the interaction of Stat1 mRNA with RBP CAPRIN1, promoting IFN- $\gamma-S T A T 1-m e d i a t e d ~ M 1$ polarization (Xu et al., 2019). The nuclear Malat1 suppresses IFN production by inhibiting the cleavage of TDP43 to TDP35, which would stabilize the Rbck1 pre-mRNA and promote the proteasomal degradation of IRF3 upon viral infection (Liu et al., 2020). The upregulated Inc-Dpf3 by CCR7 stimulation could directly bind to and suppress the HIF-1a-dependent transcription, which restrained CCR7-mediated DC migration by inhibiting its glycolytic metabolism and migratory capacity (Liu et al., 2019a). LncRNA-GM promoted the macrophage antiviral responses by binding to and relieving the suppression of GSTM1 on TBK1 activity (Wang et al., 2020). It was unknown whether the IncRNA expression is specifically induced by certain stimulation or controlled by the classic signaling pathways. Here, a number of IncRNAs were found to be downstream of the Notch pathway which negatively affected the NICD-mediated NF-KB activation, thus reprogramming macrophage polarization. Mechanistically, Inc-ip65 was directly bound to the protein domains of p65 that were adjacent to its phosphorylation sites, whose conformational hindrance might disturb the NICD-bridged interaction of p65 with IKK $\beta$, and block the S276, S529 and S536 phosphorylation of p65. This shed light on a new mechanism of IncRNA in immunoregulation.

In general, viral-bacterial co-infections would aggravate the patient's medical condition and increase disease mortality (Bakaletz, 2017). IAV dysregulated host immune responses and damage the respiratory mucosal barrier, which supported bacterial growth, adherence and invasion into normally sterile sites, thus resulting in overwhelming infection with cytokine storm syndrome (Maclntyre et al., 2018; Sharifipour et al., 2020). Besides, viral infection might also augment host inflammation in several 
autoimmune diseases (Getts et al., 2013). Enterovirus triggered trained macrophage immunity that could more promptly drive naïve T helper cells toward Th2 and Th17 cell differentiation when exposed to mites, predisposing hosts to allergic asthma (Chen et al., 2021). Conversely, we found that the pretreatment of HTNV might improve mice condition during secondary bacterial sepsis challenge (Figure 3 and S5), and the mechanisms were involved with the anti-M1 phenotype of macrophage reshaped by HTNV at the late infection stage. This revealed a different symbiotic relationship between viruses and bacteria in nature, especially for those zoonotic pathogens. In fact, the beneficial effects on hosts caused by the commensalism of different pathogens could be detected under parasite-bacteria co-infection circumstances. Concomitant Infection of S. mansoni and H. pylori restricted the liver fibrotic responses by misdirecting antigen-experienced $\mathrm{CXCR}^{+} \mathrm{T}$ cells to the liver (Bhattacharjee et al., 2019). However, it still remained further investigation for whether viral infection, no matter acute or chronic, could benefit the hosts against other pathogen invasion or autoimmune disorders.

Moreover, two potential intervention tactics for HFRS were proposed. Previous studies have reported multiple negative feedback loops against exorbitant immune activation, such as vascular endothelial growth factor receptor-3 (VEGFR-3)-mediated anti-inflammatory effects by enhancing SOCS1 expression and inhibiting TLR4-NF-KB pathway during endotoxin shock (Zhang et al., 2014b), and ubiquitin-specific peptidase 38 (UPS38)-mediated anti-IFN effects by degrading TBK1 during viral infection (Lin et al., 2016). As these antagonistic factors would be endogenously upregulated along the pathogenic process, exogenous supplementation of them could obtain limited curative effects. In this study, the murinespecific IncRNAs were found to hinder immoderate inflammation both in mice and human macrophages, which suggested that applying the negative regulons from other species might be a potential therapy choice for patients. Besides, the non-neutralizing antibody against NP could incompletely improve host conditions in HTNV-infected neonatal models, possibly by attenuating macrophage-mediated inflammation, which would also prolong the effective therapeutic window of neutralizing antibodies (Figure 8S-U). This indicated combination of antibodies against different viral proteins, not only the neutralizing antibodies, might achieve better clinical efficacy.

Collectively, we demonstrated the differential macrophage responses against HTNV infection in mice versus humans, and the late-phase inactivation of inflammatory macrophages in mice prohibited the cytokine storm and protected them from secondary endotoxin sepsis. Murine Notch signaling dynamically rewired the macrophage phenotype by producing NICD and IncRNAs, of which Inc-ip65 could inhibit the NF-KB pathway and impel an anti-M1 status. Blocking Notch activation to prevent M1 activation at the early stage, or applying Inc-ip65 to restrain hyperactivation of M1 at the late stage, might be effective for the control of inflammation and NF-KB -associated autoimmune diseases.

\section{Limitations Of The Study}

The murine Notch signaling was found to be dynamically activated during HTNV infection, while it remained unclear which factor determined the accumulation of NICD in the cytoplasm at the early stage but translocation of NICD in the nucleus at the late stage (Figure 4 and S7). There existed a possibility 
that the NICD function was manipulated by HTNV rather than cytokines, as we previously showed that HTNV could dynamically influence host autophagy flux with different viral proteins (Wang et al., 2019). To note, a recent study indicated viruses could subvert macrophage identity, which meant that the virusinfected and bystander macrophages would maintain distinctive immunophenotypes (Baasch et al., 2021). Here, the virus-infected and bystander macrophages were not distinguished but detected as an integral group. Though most macrophages were infected by HTNV (Figure S9C) and their regulatory role on inflammation was emphasized in this study, it was meaningful to explore whether the virus-infected and bystander macrophages showed a distinctive reprogramming process.

Beyond the pro- or anti-inflammatory role, the antiviral capacity of macrophages was also of great importance (Li et al., 2018; Raftery et al., 2020; Scholz et al., 2017). There did exist a racing game between viruses and host immune cells, as macrophages could effectively restrain HTNV infection (Figure 2), while this limitation seemed to be broken if several IncRNAs were intervened (Figure 6 and 7 ) or the challenge dose was increased (Figure S9C). Lnc-ip65 and other murine-specific IncRNAs negatively regulated the type I IFN production (Figure 8D, 8G, 8J and S9B) and Stat1 phosphorylation (Figure 6A), suggesting that they might influence the IFN signaling. Silencing Inc-ip65 would promote the antiviral ability in vitro (Figure 6), while the results seemed to be contrary in Inc-ip65 $5^{-/-}$mice (Figure 7). One possibility is that some other negative regulatory factors of antiviral response might be provoked as compensatory effects in the Inc-ip65 deficient mice. Alternatively, as we generated the conventional Incip $65^{-/-}$mice with CRISPR/Cas9 technology Inc-ip65, it could not exclude the possibility that loss function of Inc-ip65 in non-immune cells would promote viral replication. Whether Inc-ip65 could regulate the host antiviral immunity, such as IFN signaling or IFN-independent pathway, awaits clari[cation.

Finally, it remains further investigation about whether the murine-specific IncRNAs could be applied to prevent the hyperactivation of inflammatory monocytes or macrophages in HFRS patients. On the one hand, exogenous RNAs could be recognized by host PRRs and degraded before they perform the antiinflammatory function. On the other hand, it is crucial to ascertain the suitable therapeutic window, as premature treatment with IncRNAs would affect viral clearance by disturbing host immune responses, and delayed remedy might not effectively attenuate systemic inflammation.

\section{Declarations}

\section{ACKNOWLEDGMENTS}

The authors thank Hongyan Qin and Hua Han for providing the RBP-JCKO and NICD ${ }^{\text {STOP-floxed }}$ mice and guidance for flow cytometry assays, as well as Jing Ye for technical and analytical support for detecting mice tissue pathogenic injuries. We further thank Zhansheng Jia, Jianqi Lian and Wen Yin for assisting the clinical sample and medical record collection, as well as Pengbo Yu for $A$. agrarius mice capture. The authors acknowledge support from the National Natural Science Foundation of China (82172272, 81671994 and 31970148), Key Research and Development Program of Shaanxi Province (2021ZDLSF0102). The graphical abstract has been created with BioRender.com. 


\section{AUTHOR CONTRIBUTIONS}

F.Z., Y.L., and H.M. conceptualized the study. X.Z. and Z.X. supervised the research and provided excellent scientific discussion when this study encountered with problems. H.M. and Y.L. designed the methodology. H.M., Y.Y. and T.N. performed the experiments. R.Y. and S.Y. identified and bred the transgenic mice. J.W. and M.L. took charge of the field mice capture. H.L. and W.Y. collected the clinical samples and medical records of patients. H.Z. and X.L. constructed the protein and IncRNA mutants, as well as other vectors and the VLP of HTNV. L.C. and L.Z. contributed the reagents and analytical tools. L.L., Z.X. and X.Z. conducted the pathology analysis. F.Z. and X.L. acquired the funding. H.M., Y.Y. and T.N. analyzed the data and wrote the manuscript with input from all the authors.

\section{DECLARATION OF INTERESTS}

The authors declare no competing interests.

\section{References}

1. Abudurexiti, A., Adkins, S., Alioto, D., Alkhovsky, S.V., Avšič-Županc, T., Ballinger, M.J., Bente, D.A., Beer, M., Bergeron, É., Blair, C.D., et al. (2019). Taxonomy of the order Bunyavirales: update 2019. Arch Virol 164, 1949-1965.

2. Angulo, J., Martínez-Valdebenito, C., Marco, C., Galeno, H., Villagra, E., Vera, L., Lagos, N., Becerra, N., Mora, J., Bermúdez, A., et al. (2017). Serum levels of interleukin-6 are linked to the severity of the disease caused by Andes Virus. PLoS Negl Trop Dis 11, e0005757.

3. Armaos, A., Colantoni, A., Proietti, G., Rupert, J., and Tartaglia, G.G. (2021). catRAPID omics v2.0: going deeper and wider in the prediction of protein-RNA interactions. Nucleic Acids Res 49, W72-W79.

4. Au, R.Y., Jedlicka, A.E., Li, W., Pekosz, A., and Klein, S.L. (2010). Seoul virus suppresses NF-kappaBmediated inflammatory responses of antigen presenting cells from Norway rats. Virology 400,115127.

5. Baasch, S., Giansanti, P., Kolter, J., Riedl, A., Forde, A.J., Runge, S., Zenke, S., Elling, R., Halenius, A., Brabletz, S., et al. (2021). Cytomegalovirus subverts macrophage identity. Cell 184, 3774-3793 e3725.

6. Bakaletz, L.O. (2017). Viral-bacterial co-infections in the respiratory tract. Curr Opin Microbiol 35, 3035.

7. Bhattacharjee, S., Mejías-Luque, R., Loffredo-Verde, E., Toska, A., Flossdorf, M., Gerhard, M., and Prazeres da Costa, C. (2019). Concomitant Infection of S. mansoni and H. pylori Promotes Promiscuity of Antigen-Experienced Cells and Primes the Liver for a Lower Fibrotic Response. Cell Rep 28, 231-244 e235.

8. Boldin, M.P., and Baltimore, D. (2012). MicroRNAs, new effectors and regulators of NF-KB. Immunol Rev 246, 205-220.

9. Bourgognon, M., Klippstein, R., and Al-Jamal, K.T. (2015). Kupffer Cell Isolation for Nanoparticle Toxicity Testing. J Vis Exp, e52989. 
10. Braun, M., Björkström, N.K., Gupta, S., Sundström, K., Ahlm, C., Klingström, J., and Ljunggren, H.G. (2014). NK cell activation in human hantavirus infection explained by virus-induced IL-15/IL15Ra expression. PLoS Pathog 10, e1004521.

11. Brocato, R.L., and Hooper, J.W. (2019). Progress on the Prevention and Treatment of Hantavirus Disease. Viruses 11.

12. Carpenter, S., Aiello, D., Atianand, M.K., Ricci, E.P., Gandhi, P., Hall, L.L., Byron, M., Monks, B., HenryBezy, M., Lawrence, J.B., et al. (2013). A long noncoding RNA mediates both activation and repression of immune response genes. Science 341, 789-792.

13. Chabriat, H., Joutel, A., Dichgans, M., Tournier-Lasserve, E., and Bousser, M.G. (2009). Cadasil. Lancet Neurol 8,643-653.

14. Chen, H., Hu, X., Li, R., Liu, B., Zheng, X., Fang, Z., Chen, L., Chen, W., Min, L., and Hu, S. (2020a). LncRNA THRIL aggravates sepsis-induced acute lung injury by regulating miR-424/ROCK2 axis. Mol Immunol 126, 111-119.

15. Chen, P.C., Shao, Y.T., Hsieh, M.H., Kao, H.F., Kuo, W.S., Wang, S.M., Chen, S.H., Wu, L.S.H., Tsai, H.J., and Wang, J.Y. (2021). Early-life EV-A71 infection augments allergen-induced airway inflammation in asthma through trained macrophage immunity. Cell Mol Immunol 18, 472-483.

16. Chen, Q.Z., Luo, F., Lu, M.X., Li, N., Teng, Y., Huang, Q.L., Zhu, N., Wang, G.Y., Yue, M., Zhang, Y., et al. (2017a). HTNV-induced upregulation of miR-146a in HUVECs promotes viral infection by modulating pro-inflammatory cytokine release. Biochem Biophys Res Commun 493, 807-813.

17. Chen, Q.Z., Wang, X., Luo, F., Li, N., Zhu, N., Lu, S., Zan, Y.X., Zhong, C.J., Wang, M.R., Hu, H.T., et al. (2020b). HTNV Sensitizes Host Toward TRAIL-Mediated Apoptosis-A Pivotal Anti-hantaviral Role of TRAIL. Front Immunol 11, 1072.

18. Chen, Y.G., Satpathy, A.T., and Chang, H.Y. (2017b). Gene regulation in the immune system by long noncoding RNAs. Nat Immunol 18, 962-972.

19. Cheng, L.F., Wang, F., Zhang, L., Yu, L., Ye, W., Liu, Z.Y., Ying, Q.K., Wu, X.A., Xu, Z.K., and Zhang, F.L. (2016). Incorporation of GM-CSF or CD40L Enhances the Immunogenicity of Hantaan Virus-Like Particles. Front Cell Infect Microbiol 6, 185.

20. Cole, S.L., Dunning, J., Kok, W.L., Benam, K.H., Benlahrech, A., Repapi, E., Martinez, F.O., Drumright, L., Powell, T.J., Bennett, M., et al. (2017). M1-like monocytes are a major immunological determinant of severity in previously healthy adults with life-threatening influenza. JCI Insight 2, e91868.

21. Cook, K.B., Kazan, H., Zuberi, K., Morris, Q., and Hughes, T.R. (2011). RBPDB: a database of RNAbinding specificities. Nucleic Acids Res 39, D301-308.

22. Douglas, K.O., Samuels, T.A., and Gittens-St Hilaire, M. (2019). Serum LPS Associated with Hantavirus and Dengue Disease Severity in Barbados. Viruses 11.

23. Easterbrook, J.D., and Klein, S.L. (2008). Immunological mechanisms mediating hantavirus persistence in rodent reservoirs. PLoS Pathog 4, e1000172.

24. Easterbrook, J.D., Zink, M.C., and Klein, S.L. (2007). Regulatory T cells enhance persistence of the zoonotic pathogen Seoul virus in its reservoir host. Proc Natl Acad Sci U S A 104, 15502-15507. 
25. Fajgenbaum, D.C., and June, C.H. (2020). Cytokine Storm. N Engl J Med 383, 2255-2273.

26. Fan, X., Deng, H., Sang, J., Li, N., Zhang, X., Han, Q., and Liu, Z. (2018a). High Serum Procalcitonin Concentrations in Patients With Hemorrhagic Fever With Renal Syndrome Caused by Hantaan Virus. Front Cell Infect Microbiol 8, 129.

27. Fan, X., Liu, Z., Fu, S., Sang, J., Deng, H., Li, F., Zhang, X., Li, N., Han, Q., and Liu, Z. (2018b). Platelet Distribution Width at First Day of Hospital Admission in Patients with Hemorrhagic Fever with Renal Syndrome Caused by Hantaan Virus May Predict Disease Severity and Critical Patients' Survival. Dis Markers 2018, 9701619.

28. Fanucchi, S., Fok, E.T., Dalla, E., Shibayama, Y., Börner, K., Chang, E.Y., Stoychev, S., Imakaev, M., Grimm, D., Wang, K.C., et al. (2019). Immune genes are primed for robust transcription by proximal long noncoding RNAs located in nuclear compartments. Nat Genet 51, 138-150.

29. Ferrandino, F., Grazioli, P., Bellavia, D., Campese, A.F., Screpanti, I., and Felli, M.P. (2018). Notch and NF-kB: Coach and Players of Regulatory T-Cell Response in Cancer. Front Immunol 9, 2165.

30. Foldi, J., Shang, Y., Zhao, B., Ivashkiv, L.B., and Hu, X. (2016). RBP-J is required for M2 macrophage polarization in response to chitin and mediates expression of a subset of $\mathrm{M} 2$ genes. Protein Cell 7 , 201-209.

31. Getts, D.R., Chastain, E.M., Terry, R.L., and Miller, S.D. (2013). Virus infection, antiviral immunity, and autoimmunity. Immunol Rev 255, 197-209.

32. Ginhoux, F., and Guilliams, M. (2016). Tissue-Resident Macrophage Ontogeny and Homeostasis. Immunity 44, 439-449.

33. Ginhoux, F., and Jung, S. (2014). Monocytes and macrophages: developmental pathways and tissue homeostasis. Nat Rev Immunol 14, 392-404.

34. Guilliams, M., Mildner, A., and Yona, S. (2018). Developmental and Functional Heterogeneity of Monocytes. Immunity 49, 595-613.

35. Guivier, E., Galan, M., Salvador, A.R., Xuéreb, A., Chaval, Y., Olsson, G.E., Essbauer, S., Henttonen, H., Voutilainen, L., Cosson, J.F., et al. (2010). Tnf-a expression and promoter sequences reflect the balance of tolerance/resistance to Puumala hantavirus infection in European bank vole populations. Infect Genet Evol 10, 1208-1217.

36. Guo, J.C., Fang, S.S., Wu, Y., Zhang, J.H., Chen, Y., Liu, J., Wu, B., Wu, J.R., Li, E.M., Xu, L.Y., et al. (2019). CNIT: a fast and accurate web tool for identifying protein-coding and long non-coding transcripts based on intrinsic sequence composition. Nucleic Acids Res 47, W516-W522.

37. Gupta, S., Braun, M., Tischler, N.D., Stoltz, M., Sundström, K.B., Björkström, N.K., Ljunggren, H.G., and Klingström, J. (2013). Hantavirus-infection confers resistance to cytotoxic lymphocyte-mediated apoptosis. PLoS Pathog 9, e1003272.

38. Haeussler, M., Zweig, A.S., Tyner, C., Speir, M.L., Rosenbloom, K.R., Raney, B.J., Lee, C.M., Lee, B.T., Hinrichs, A.S., Gonzalez, J.N., et al. (2019). The UCSC Genome Browser database: 2019 update. Nucleic Acids Res 47, D853-D858. 
39. Hannah, M.F., Bajic, V.B., and Klein, S.L. (2008). Sex differences in the recognition of and innate antiviral responses to Seoul virus in Norway rats. Brain Behav Immun 22, 503-516.

40. Haschemi, A., Kosma, P., Gille, L., Evans, C.R., Burant, C.F., Starkl, P., Knapp, B., Haas, R., Schmid, J.A., Jandl, C., et al. (2012). The sedoheptulose kinase CARKL directs macrophage polarization through control of glucose metabolism. Cell Metab 15, 813-826.

41. Hu, X., Chung, A.Y., Wu, I., Foldi, J., Chen, J., Ji, J.D., Tateya, T., Kang, Y.J., Han, J., Gessler, M., et al. (2008). Integrated regulation of Toll-like receptor responses by Notch and interferon-gamma pathways. Immunity 29,691-703.

42. Imamura, K., Imamachi, N., Akizuki, G., Kumakura, M., Kawaguchi, A., Nagata, K., Kato, A., Kawaguchi, Y., Sato, H., Yoneda, M., et al. (2014). Long noncoding RNA NEAT1-dependent SFPQ relocation from promoter region to paraspeckle mediates IL8 expression upon immune stimuli. Mol Cell 53,393406.

43. Italiani, P., and Boraschi, D. (2014). From Monocytes to M1/M2 Macrophages: Phenotypical vs. Functional Differentiation. Front Immunol 5, 514.

44. Ito, T., Allen, R.M., Carson, W.F.t., Schaller, M., Cavassani, K.A., Hogaboam, C.M., Lukacs, N.W., Matsukawa, A., and Kunkel, S.L. (2011). The critical role of Notch ligand Delta-like 1 in the pathogenesis of influenza A virus (H1N1) infection. PLoS Pathog 7, e1002341.

45. Jiang, H., Zheng, X., Wang, L., Du, H., Wang, P., and Bai, X. (2017). Hantavirus infection: a global zoonotic challenge. Virol Sin 32, 32-43.

46. Jiang, M., Zhang, S., Yang, Z., Lin, H., Zhu, J., Liu, L., Wang, W., Liu, S., Liu, W., Ma, Y., et al. (2018). Self-Recognition of an Inducible Host IncRNA by RIG-I Feedback Restricts Innate Immune Response. Cell 173, 906-919 e913.

47. Kelly, B., and O'Neill, L.A. (2015). Metabolic reprogramming in macrophages and dendritic cells in innate immunity. Cell Res 25, 771-784.

48. Khaiboullina, S.F., Levis, S., Morzunov, S.P., Martynova, E.V., Anokhin, V.A., Gusev, O.A., St Jeor, S.C., Lombardi, V.C., and Rizvanov, A.A. (2017). Serum Cytokine Profiles Differentiating Hemorrhagic Fever with Renal Syndrome and Hantavirus Pulmonary Syndrome. Front Immunol 8, 567.

49. Khaiboullina, S.F., Martynova, E.V., Khamidullina, Z.L., Lapteva, E.V., Nikolaeva, I.V., Anokhin, V.V., Lombardi, V.C., and Rizvanov, A.A. (2014). Upregulation of IFN- $y$ and IL-12 is associated with a milder form of hantavirus hemorrhagic fever with renal syndrome. Eur J Clin Microbiol Infect Dis 33, 21492156.

50. Kim, J.A., Kim, W.K., No, J.S., Lee, S.H., Lee, S.Y., Kim, J.H., Kho, J.H., Lee, D., Song, D.H., Gu, S.H., et al. (2016). Genetic Diversity and Reassortment of Hantaan Virus Tripartite RNA Genomes in Nature, the Republic of Korea. PLoS Negl Trop Dis 10, e0004650.

51. Koivula, T.T., Tuulasvaara, A., Hetemäki, I., Mäkelä, S.M., Mustonen, J., Sironen, T., Vaheri, A., and Arstila, T.P. (2014). Regulatory T cell response correlates with the severity of human hantavirus infection. J Infect 68, 387-394. 
52. Kopan, R., and llagan, M.X. (2009). The canonical Notch signaling pathway: unfolding the activation mechanism. Cell 137, 216-233.

53. Kumar, V., Palermo, R., Talora, C., Campese, A.F., Checquolo, S., Bellavia, D., Tottone, L., Testa, G., Miele, E., Indraccolo, S., et al. (2014). Notch and NF-kB signaling pathways regulate miR-223/FBXW7 axis in T-cell acute lymphoblastic leukemia. Leukemia 28, 2324-2335.

54. Kusnadi, A., Park, S.H., Yuan, R., Pannellini, T., Giannopoulou, E., Oliver, D., Lu, T., Park-Min, K.H., and Ivashkiv, L.B. (2019). The Cytokine TNF Promotes Transcription Factor SREBP Activity and Binding to Inflammatory Genes to Activate Macrophages and Limit Tissue Repair. Immunity 51, 241-257 e249.

55. Lasso, G., Mayer, S.V., Winkelmann, E.R., Chu, T., Elliot, O., Patino-Galindo, J.A., Park, K., Rabadan, R., Honig, B., and Shapira, S.D. (2019). A Structure-Informed Atlas of Human-Virus Interactions. Cell 178, 1526-1541 e1516.

56. Lee, H.W., Lee, P.W., Baek, L.J., Song, C.K., and Seong, I.W. (1981). Intraspecific transmission of Hantaan virus, etiologic agent of Korean hemorrhagic fever, in the rodent Apodemus agrarius. Am J Trop Med Hyg 30, 1106-1112.

57. Li, P.Z., Li, J.Z., Li, M., Gong, J.P., and He, K. (2014a). An efficient method to isolate and culture mouse Kupffer cells. Immunol Lett 158, 52-56.

58. Li, W., and Klein, S.L. (2012). Seoul virus-infected rat lung endothelial cells and alveolar macrophages differ in their ability to support virus replication and induce regulatory $T$ cell phenotypes. $J$ Virol 86 , $11845-11855$.

59. Li, X., Du, N., Xu, G., Zhang, P., Dang, R., Jiang, Y., and Zhang, K. (2018). Expression of CD206 and CD163 on intermediate CD14(++)CD16(+) monocytes are increased in hemorrhagic fever with renal syndrome and are correlated with disease severity. Virus Res 253, 92-102.

60. Li, Y., Wu, S., Pu, J., Huang, X., and Zhang, P. (2015). Dengue virus up-regulates expression of notch ligands DII1 and DII4 through interferon- $\beta$ signalling pathway. Immunology $144,127-138$.

61. Li, Z., Chao, T.C., Chang, K.Y., Lin, N., Patil, V.S., Shimizu, C., Head, S.R., Burns, J.C., and Rana, T.M. (2014b). The long noncoding RNA THRIL regulates TNFa expression through its interaction with hnRNPL. Proc Natl Acad Sci U S A 111, 1002-1007.

62. Lin, H., Jiang, M., Liu, L., Yang, Z., Ma, Z., Liu, S., Ma, Y., Zhang, L., and Cao, X. (2019). The long noncoding RNA Lnczc3h7a promotes a TRIM25-mediated RIG-I antiviral innate immune response. Nat Immunol 20,812-823.

63. Lin, M., Zhao, Z., Yang, Z., Meng, Q., Tan, P., Xie, W., Qin, Y., Wang, R.F., and Cui, J. (2016). USP38 Inhibits Type I Interferon Signaling by Editing TBK1 Ubiquitination through NLRP4 Signalosome. Mol Cell 64, 267-281.

64. Liu, B., Sun, L., Liu, Q., Gong, C., Yao, Y., Lv, X., Lin, L., Yao, H., Su, F., Li, D., et al. (2015). A cytoplasmic NF-KB interacting long noncoding RNA blocks ІкB phosphorylation and suppresses breast cancer metastasis. Cancer Cell 27, 370-381.

65. Liu, J., Zhang, X., Chen, K., Cheng, Y., Liu, S., Xia, M., Chen, Y., Zhu, H., Li, Z., and Cao, X. (2019a). CCR7 Chemokine Receptor-Inducible Inc-Dpf3 Restrains Dendritic Cell Migration by Inhibiting HIF-1a- 
Mediated Glycolysis. Immunity 50, 600-615 e615.

66. Liu, R., Ma, H., Shu, J., Zhang, Q., Han, M., Liu, Z., Jin, X., Zhang, F., and Wu, X. (2019b). Vaccines and Therapeutics Against Hantaviruses. Front Microbiol 10, 2989.

67. Liu, W., Wang, Z., Liu, L., Yang, Z., Liu, S., Ma, Z., Liu, Y., Ma, Y., Zhang, L., Zhang, X., et al. (2020). LncRNA Malat1 inhibition of TDP43 cleavage suppresses IRF3-initiated antiviral innate immunity. Proc Natl Acad Sci U S A 117, 23695-23706.

68. Lv, L.L., Feng, Y., Wu, M., Wang, B., Li, Z.L., Zhong, X., Wu, W.J., Chen, J., Ni, H.F., Tang, T.T., et al. (2020). Exosomal miRNA-19b-3p of tubular epithelial cells promotes M1 macrophage activation in kidney injury. Cell Death Differ 27, 210-226.

69. Ma, H., Han, P., Ye, W., Chen, H., Zheng, X., Cheng, L., Zhang, L., Yu, L., Wu, X., Xu, Z., et al. (2017a). The Long Noncoding RNA NEAT1 Exerts Antihantaviral Effects by Acting as Positive Feedback for RIG-I Signaling. J Virol 91.

70. Ma, H.W., Ye, W., Chen, H.S., Nie, T.J., Cheng, L.F., Zhang, L., Han, P.J., Wu, X.A., Xu, Z.K., Lei, Y.F., et al. (2017b). In-Cell Western Assays to Evaluate Hantaan Virus Replication as a Novel Approach to Screen Antiviral Molecules and Detect Neutralizing Antibody Titers. Front Cell Infect Microbiol 7, 269.

71. Ma, Y., Yuan, B., Zhuang, R., Zhang, Y., Liu, B., Zhang, C., Zhang, Y., Yu, H., Yi, J., Yang, A., et al. (2015). Hantaan virus infection induces both Th1 and ThGranzyme B+ cell immune responses that associated with viral control and clinical outcome in humans. PLoS Pathog 11, e1004788.

72. Maclntyre, C.R., Chughtai, A.A., Barnes, M., Ridda, I., Seale, H., Toms, R., and Heywood, A. (2018). The role of pneumonia and secondary bacterial infection in fatal and serious outcomes of pandemic influenza a(H1N1)pdm09. BMC Infect Dis 18,637.

73. Maniati, E., Bossard, M., Cook, N., Candido, J.B., Emami-Shahri, N., Nedospasov, S.A., Balkwill, F.R., Tuveson, D.A., and Hagemann, T. (2011). Crosstalk between the canonical NF-kB and Notch signaling pathways inhibits Ppary expression and promotes pancreatic cancer progression in mice. J Clin Invest $121,4685-4699$.

74. Martínez, V.P., Di Paola, N., Alonso, D.O., Pérez-Sautu, U., Bellomo, C.M., Iglesias, A.A., Coelho, R.M., López, B., Periolo, N., Larson, P.A., et al. (2020). "Super-Spreaders" and Person-to-Person Transmission of Andes Virus in Argentina. N Engl J Med 383, 2230-2241.

75. Mathews, D.H., Disney, M.D., Childs, J.L., Schroeder, S.J., Zuker, M., and Turner, D.H. (2004). Incorporating chemical modification constraints into a dynamic programming algorithm for prediction of RNA secondary structure. Proc Natl Acad Sci U S A 101, 7287-7292.

76. Merad, M., and Martin, J.C. (2020). Pathological inflammation in patients with COVID-19: a key role for monocytes and macrophages. Nat Rev Immunol 20,355-362.

77. Mukherjee, S., Akbar, I., Kumari, B., Vrati, S., Basu, A., and Banerjee, A. (2019). Japanese Encephalitis Virus-induced let-7a/b interacted with the NOTCH-TLR7 pathway in microglia and facilitated neuronal death via caspase activation. J Neurochem 149, 518-534.

78. Murray, P.J., Allen, J.E., Biswas, S.K., Fisher, E.A., Gilroy, D.W., Goerdt, S., Gordon, S., Hamilton, J.A., Ivashkiv, L.B., Lawrence, T., et al. (2014). Macrophage activation and polarization: nomenclature and 
experimental guidelines. Immunity 41, 14-20.

79. Niikura, M., Maeda, A., Ikegami, T., Saijo, M., Kurane, I., and Morikawa, S. (2004). Modification of endothelial cell functions by Hantaan virus infection: prolonged hyper-permeability induced by TNFalpha of hantaan virus-infected endothelial cell monolayers. Arch Virol 149, 1279-1292.

80. No, J.S., Kim, W.K., Cho, S., Lee, S.H., Kim, J.A., Lee, D., Song, D.H., Gu, S.H., Jeong, S.T., Wiley, M.R., et al. (2019). Comparison of targeted next-generation sequencing for whole-genome sequencing of Hantaan orthohantavirus in Apodemus agrarius lung tissues. Sci Rep 9, 16631.

81. Platanitis, E., and Decker, T. (2018). Regulatory Networks Involving STATs, IRFs, and NFkB in Inflammation. Front Immunol 9, 2542.

82. Plekhova, N.G., Somova, L.M., Slonova, R.A., Companets, G.G., Luk'yanova, V.V., and Yakubovich, N.V. (2005). Metabolic activity of macrophages infected with hantavirus, an agent of hemorrhagic fever with renal syndrome. Biochemistry (Mosc) 70, 990-997.

83. Priem, D., van Loo, G., and Bertrand, M.J.M. (2020). A20 and Cell Death-driven Inflammation. Trends Immunol 41, 421-435.

84. Radtke, F., Fasnacht, N., and Macdonald, H.R. (2010). Notch signaling in the immune system. Immunity 32, 14-27.

85. Raftery, M.J., Lalwani, P., Lütteke, N., Kobak, L., Giese, T., Ulrich, R.G., Radosa, L., Krüger, D.H., and Schönrich, G. (2020). Replication in the Mononuclear Phagocyte System (MPS) as a Determinant of Hantavirus Pathogenicity. Front Cell Infect Microbiol 10, 281.

86. Rahman, M.M., and McFadden, G. (2011). Modulation of NF-KB signalling by microbial pathogens. Nat Rev Microbiol 9, 291-306.

87. Ruland, J. (2011). Return to homeostasis: downregulation of NF-KB responses. Nat Immunol 12, 709-714.

88. Saha, B., Kodys, K., and Szabo, G. (2016). Hepatitis C Virus-Induced Monocyte Differentiation Into Polarized M2 Macrophages Promotes Stellate Cell Activation via TGF- $\beta$. Cell Mol Gastroenterol Hepatol 2, 302-316 e308.

89. Saksida, A., Wraber, B., and Avšič-Županc, T. (2011). Serum levels of inflammatory and regulatory cytokines in patients with hemorrhagic fever with renal syndrome. BMC Infect Dis 11, 142.

90. Santoro, M.G., Rossi, A., and Amici, C. (2003). NF-kappaB and virus infection: who controls whom. EMBO J 22, 2552-2560.

91. Scholz, S., Baharom, F., Rankin, G., Maleki, K.T., Gupta, S., Vangeti, S., Pourazar, J., Discacciati, A., Höijer, J., Bottai, M., et al. (2017). Human hantavirus infection elicits pronounced redistribution of mononuclear phagocytes in peripheral blood and airways. PLoS Pathog 13, e1006462.

92. Schönrich, G., Rang, A., Lütteke, N., Raftery, M.J., Charbonnel, N., and Ulrich, R.G. (2008). Hantavirusinduced immunity in rodent reservoirs and humans. Immunol Rev 225, 163-189.

93. Schountz, T., and Prescott, J. (2014). Hantavirus immunology of rodent reservoirs: current status and future directions. Viruses 6,1317-1335. 
94. Schountz, T., Prescott, J., Cogswell, A.C., Oko, L., Mirowsky-Garcia, K., Galvez, A.P., and Hjelle, B. (2007). Regulatory T cell-like responses in deer mice persistently infected with Sin Nombre virus. Proc Natl Acad Sci U S A 104, 15496-15501.

95. Shang, Y., Smith, S., and Hu, X. (2016). Role of Notch signaling in regulating innate immunity and inflammation in health and disease. Protein Cell 7, 159-174.

96. Shang, Z., Yu, J., Sun, L., Tian, J., Zhu, S., Zhang, B., Dong, Q., Jiang, N., Flores-Morales, A., Chang, C., et al. (2019). LncRNA PCAT1 activates AKT and NF-KB signaling in castration-resistant prostate cancer by regulating the PHLPP/FKBP51/IKKa complex. Nucleic Acids Res 47, 4211-4225.

97. Sharifipour, E., Shams, S., Esmkhani, M., Khodadadi, J., Fotouhi-Ardakani, R., Koohpaei, A., Doosti, Z., and Ej Golzari, S. (2020). Evaluation of bacterial co-infections of the respiratory tract in COVID-19 patients admitted to ICU. BMC Infect Dis 20,646.

98. Solà-Riera, C., Gupta, S., Maleki, K.T., González-Rodriguez, P., Saidi, D., Zimmer, C.L., Vangeti, S., Rivino, L., Leo, Y.S., Lye, D.C., et al. (2019). Hantavirus Inhibits TRAIL-Mediated Killing of Infected Cells by Downregulating Death Receptor 5. Cell Rep 28, 2124-2139 e2126.

99. Statello, L., Guo, C.J., Chen, L.L., and Huarte, M. (2021). Gene regulation by long non-coding RNAs and its biological functions. Nat Rev Mol Cell Biol 22, 96-118.

100. Szklarczyk, D., Gable, A.L., Nastou, K.C., Lyon, D., Kirsch, R., Pyysalo, S., Doncheva, N.T., Legeay, M., Fang, T., Bork, P., et al. (2021). The STRING database in 2021: customizable protein-protein networks, and functional characterization of user-uploaded gene/measurement sets. Nucleic Acids Res 49, D605-D612.

101. Taylor, S.L., Frias-Staheli, N., García-Sastre, A., and Schmaljohn, C.S. (2009a). Hantaan virus nucleocapsid protein binds to importin alpha proteins and inhibits tumor necrosis factor alphainduced activation of nuclear factor kappa B. J Virol 83, 1271-1279.

102. Taylor, S.L., Krempel, R.L., and Schmaljohn, C.S. (2009b). Inhibition of TNF-alpha-induced activation of NF-kappaB by hantavirus nucleocapsid proteins. Ann N Y Acad Sci 1171 Suppl 1, E86-93.

103. Taylor, S.L., Wahl-Jensen, V., Copeland, A.M., Jahrling, P.B., and Schmaljohn, C.S. (2013). Endothelial cell permeability during hantavirus infection involves factor XII-dependent increased activation of the kallikrein-kinin system. PLoS Pathog 9, e1003470.

104. Terajima, M., and Ennis, F.A. (2011). T cells and pathogenesis of hantavirus cardiopulmonary syndrome and hemorrhagic fever with renal syndrome. Viruses 3, 1059-1073.

105. Tian, H., Yu, P., Cazelles, B., Xu, L., Tan, H., Yang, J., Huang, S., Xu, B., Cai, J., Ma, C., et al. (2017). Interannual cycles of Hantaan virus outbreaks at the human-animal interface in Central China are controlled by temperature and rainfall. Proc Natl Acad Sci U S A 114, 8041-8046.

106. Vaheri, A., Strandin, T., Hepojoki, J., Sironen, T., Henttonen, H., Mäkelä, S., and Mustonen, J. (2013). Uncovering the mysteries of hantavirus infections. Nat Rev Microbiol 11, 539-550.

107. Vera-Otarola, J., Solis, L., Lowy, F., Olguín, V., Angulo, J., Pino, K., Tischler, N.D., Otth, C., Padula, P., and López-Lastra, M. (2020). The Andes Orthohantavirus NSs Protein Antagonizes the Type I Interferon Response by Inhibiting MAVS Signaling. J Virol 94. 
108. Vitiello, M., Galdiero, M., Finamore, E., Galdiero, S., and Galdiero, M. (2012). NF-кB as a potential therapeutic target in microbial diseases. Mol Biosyst 8,1108-1120.

109. Wang, K., Ma, H., Liu, H., Ye, W., Li, Z., Cheng, L., Zhang, L., Lei, Y., Shen, L., and Zhang, F. (2019). The Glycoprotein and Nucleocapsid Protein of Hantaviruses Manipulate Autophagy Flux to Restrain Host Innate Immune Responses. Cell Rep 27, 2075-2091 e2075.

110. Wang, P., Xue, Y., Han, Y., Lin, L., Wu, C., Xu, S., Jiang, Z., Xu, J., Liu, Q., and Cao, X. (2014). The STAT3binding long noncoding RNA Inc-DC controls human dendritic cell differentiation. Science 344, 310313.

111. Wang, Y., Wang, P., Zhang, Y., Xu, J., Li, Z., Li, Z., Zhou, Z., Liu, L., and Cao, X. (2020). Decreased Expression of the Host Long-Noncoding RNA-GM Facilitates Viral Escape by Inhibiting the Kinase activity TBK1 via S-glutathionylation. Immunity 53, 1168-1181 e1167.

112. Wang, Z., Fan, P., Zhao, Y., Zhang, S., Lu, J., Xie, W., Jiang, Y., Lei, F., Xu, N., and Zhang, Y. (2017). NEAT1 modulates herpes simplex virus-1 replication by regulating viral gene transcription. Cell Mol Life Sci 74, 1117-1131.

113. Xiu, Y., Dong, Q., Fu, L., Bossler, A., Tang, X., Boyce, B., Borcherding, N., Leidinger, M., Sardina, J.L., Xue, H.H., et al. (2020). Coactivation of NF-kB and Notch signaling is sufficient to induce B-cell transformation and enables B-myeloid conversion. Blood 135, 108-120.

114. Xu, H., Jiang, Y., Xu, X., Su, X., Liu, Y., Ma, Y., Zhao, Y., Shen, Z., Huang, B., and Cao, X. (2019). Inducible degradation of IncRNA Sros1 promotes IFN-y-mediated activation of innate immune responses by stabilizing Stat1 mRNA. Nat Immunol 20,1621-1630.

115. Xu, H., Zhu, J., Smith, S., Foldi, J., Zhao, B., Chung, A.Y., Outtz, H., Kitajewski, J., Shi, C., Weber, S., et al. (2012). Notch-RBP-J signaling regulates the transcription factor IRF8 to promote inflammatory macrophage polarization. Nat Immunol 13,642-650.

116. Xu, J., Chi, F., Guo, T., Punj, V., Lee, W.N., French, S.W., and Tsukamoto, H. (2015). NOTCH reprograms mitochondrial metabolism for proinflammatory macrophage activation. J Clin Invest $125,1579-$ 1590.

117. Xu, Z., Wei, L., Wang, L., Wang, H., and Jiang, S. (2002). The in vitro and in vivo protective activity of monoclonal antibodies directed against Hantaan virus: potential application for immunotherapy and passive immunization. Biochem Biophys Res Commun 298, 552-558.

118. Yi, J., Xu, Z., Zhuang, R., Wang, J., Zhang, Y., Ma, Y., Liu, B., Zhang, Y., Zhang, C., Yan, G., et al. (2013). Hantaan virus RNA load in patients having hemorrhagic fever with renal syndrome: correlation with disease severity. J Infect Dis 207, 1457-1461.

119. Yin, D.D., Fan, F.Y., Hu, X.B., Hou, L.H., Zhang, X.P., Liu, L., Liang, Y.M., and Han, H. (2009). Notch signaling inhibits the growth of the human chronic myeloid leukemia cell line K562. Leuk Res 33, 109-114.

120. Yu, H., Jiang, W., Du, H., Xing, Y., Bai, G., Zhang, Y., Li, Y., Jiang, H., Zhang, Y., Wang, J., et al. (2014). Involvement of the Akt/NF-KB pathways in the HTNV-mediated increase of IL-6, CCL5, ICAM-1, and VCAM-1 in HUVECs. PLoS One 9, e93810. 
121. Yu, H.T., Jiang, H., Zhang, Y., Nan, X.P., Li, Y., Wang, W., Jiang, W., Yang, D.Q., Su, W.J., Wang, J.P., et al. (2012). Hantaan virus triggers TLR4-dependent innate immune responses. Viral Immunol 25, 387393.

122. Yu, Z., Zhou, N., Li, A., Chen, J., Chen, H., He, Z., Yan, F., Zhao, H., and Zhu, J. (2017). Performance assessment of the SAPS II and SOFA scoring systems in Hanta virus Hemorrhagic Fever with Renal Syndrome. Int J Infect Dis 63, 88-94.

123. Zhang, P., Cao, L., Zhou, R., Yang, X., and Wu, M. (2019). The IncRNA Neat1 promotes activation of inflammasomes in macrophages. Nat Commun 10, 1495.

124. Zhang, Q., and Cao, X. (2021). Epigenetic Remodeling in Innate Immunity and Inflammation. Annu Rev Immunol 39, 279-311.

125. Zhang, Q., Lenardo, M.J., and Baltimore, D. (2017). 30 Years of NF-kB: A Blossoming of Relevance to Human Pathobiology. Cell 168, 37-57.

126. Zhang, W., Xu, W., and Xiong, S. (2010). Blockade of Notch1 signaling alleviates murine lupus via blunting macrophage activation and M2b polarization. J Immunol 184, 6465-6478.

127. Zhang, Y., Liu, B., Ma, Y., Yi, J., Zhang, C., Zhang, Y., Xu, Z., Wang, J., Yang, K., Yang, A., et al. (2014a). Hantaan virus infection induces CXCL10 expression through TLR3, RIG-I, and MDA-5 pathways correlated with the disease severity. Mediators Inflamm 2014, 697837.

128. Zhang, Y., Lu, Y., Ma, L., Cao, X., Xiao, J., Chen, J., Jiao, S., Gao, Y., Liu, C., Duan, Z., et al. (2014b). Activation of vascular endothelial growth factor receptor-3 in macrophages restrains TLR4-NF-KB signaling and protects against endotoxin shock. Immunity 40, 501-514.

129. Zhang, Y., Zhang, C., Zhuang, R., Ma, Y., Zhang, Y., Yi, J., Yang, A., and Jin, B. (2015). IL-33/ST2 correlates with severity of haemorrhagic fever with renal syndrome and regulates the inflammatory response in Hantaan virus-infected endothelial cells. PLoS Negl Trop Dis 9, e0003514.

\section{Methods}

\section{STAR METHODS}

\section{RESOURCE AVAILABILITY}

Further information and requests for resources and reagents should be directed to and will be fulfilled by the Lead Contact, Fanglin Zhang (flzhang@fmmu.edu.cn).

\section{EXPERIMENTAL MODEL AND SUBJECT DETAILS}

\section{Human Samples and Murine Experiments}

\section{Study Participants}

This study was approved by the Institutional Review Board of Tangdu Hospital (TDLL-2016323). Peripheral blood samples and related medical records were collected from two-hundred and thirty-six 
hospitalized patients aging from 18 to 35 years old at the department of infectious disease, Tangdu Hospital from October 2016 to March 2018 (HFRS patients, n=185; Japanese encephalitis patients, acute phase, $n=15$; hepatitis $B$ patients, inactive phase without liver cirrhosis and antiviral therapy, $n=18$; hepatitis $C$ patients, inactive phase without liver cirrhosis and antiviral therapy, $n=18$ ). All patients were Han Chinese and the proportion of males to females nearly equaled 1:1. The diagnosis of HFRS or Japanese encephalitis was made based on typical symptoms and signs as well as IgM and IgG antibody positivity against HTNV or JEV in the serum as assessed by ELISA by the Department of Clinical Laboratory, Tangdu Hospital. The diagnosis of chronic HBV or HCV infection was confirmed by viral RNA detection with qRT-PCR. The definition of HFRS phases, classification of disease severity and exclusion criteria were previously described (Yi et al., 2013; Zhang et al., 2015).

The clinical blood samples of healthy individuals between the ages of 20 and 35 years were obtained from the Blood Transfusion Department of Tangdu Hospital $(n=55)$ in agreement with institutional ethics regulations. To obtain human monocyte-derived macrophages (hMDM), peripheral blood mononuclear cells (PBMC) were first enriched by Ficoll (TBDscience) from the peripheral blood density gradient centrifugation. Then human monocytes were magnetically purified from PBMC with negative screening beads (EasySep ${ }^{\mathrm{TM}}$ Human Monocyte Isolation Kit, StemCell). Finally, monocytes were primed with recombinant human macrophage colony-stimulating factor (M-CSF) $(15 \mathrm{ng} / \mathrm{ml}$, PeproTech) with medium exchange every other day for a week to generate hMDM. Alternatively, the PBMC were laid into the Petri dishes for $4 \mathrm{~h}$, and the supernatant cells were collected to acquire the monocytes removed PBMC.

\section{Animal Models}

C57BL/6J mice (six- to eight-week-old male adult mice weighing from 20-22 g, or four-day neonatal mice) were provided by the Experimental Animal Center of Air Force Medical University (AFMU). WT and transgenic mice were bred under specific pathogen-free (SPF) conditions in the animal facilities belonging to the School of Basic Medical Sciences and housed in groups of up to four mice. The Inc-ip65 deficient mice were generated using the CRISPR/Cas9 system in the C57BL/6J background, the sgRNA targeting sequences of which were shown in Figure S10A-i. The Inc-ip65 targeting vector was electroporated into C57BL/6J mouse embryonic stem (ES) cells, followed by double drug selection. Positive ES cell clones were expanded and injected into C57BL/6J blastocytes to generate chimeric offsprings. The off-spring mice were examined by genotyping PCR using the following primers as shown in Figure S10A-i.

All animals received care according to institutional guidelines, and were randomly assigned to the control or treatment group. For HTNV infection, mice were intramuscularly injected with $\mathrm{HTNV}\left(8 \times 10^{5} \mathrm{TCID}_{50} / \mathrm{g}\right.$, $8 \times 10^{6} \mathrm{TCID}_{50} / \mathrm{g}$, or $8 \times 10^{6} \mathrm{TCID}_{50} / \mathrm{g}$ of body weight) as we previously reported (Wang et al., 2019). The HTNV titer was measured by In-cell Western assays as we previously described (Ma et al., 2017b). For monocyte and macrophage depletion, mice were intraperitoneally injected with clophosome $(10 \mu \mathrm{l} / \mathrm{g}$ of body weight). For antibody treatment, mice were intraperitoneally injected with 1A8, 3D8 or 4G2 (0.25 
$\mu \mathrm{g} / \mathrm{g}$ of body weight). For the bacterial sepsis challenge, mice were intraperitoneal injected with LPS ( $5 \mathrm{mg} / \mathrm{kg}$ of body weight) or CS $(0.6 \mathrm{mg} / \mathrm{g}$ of body weight).

\section{In Vitro Experiments}

\section{Cell Culture}

HEK293, THP-1, Vero E6, bEnd.3, NIH/3T3, RAW264.7 and MH-S cells were cultured in Dulbecco's modified Eagle's medium (DMEM, Hyclone) supplemented with $10 \%(\mathrm{v} / \mathrm{v})$ fetal bovine serum (FBS, Gibco). HUVEC were co-cultured in endothelial cell medium (ECM) with Endothelial Cell Growth Supplement (ECGS) in the transwell system. The suspension THP-1 cells were stimulated by PMA (25 $\mathrm{ng} / \mathrm{ml}$, Sigma-Aldrich) for $24 \mathrm{hr}$ to differentiate into adherent macrophages. RAW264.7 and THP-1 cells stably expressing GFP-p65 and RFP-IKBa were constructed with lentivirus system and screened with puromycin and neomycin sequentially.

\section{Primary Macrophage Acquisition}

To generate the murine bone marrow-derived macrophages (mBMDM), the femur and tibia were removed from the sacrificed adult mice. The bones were first rinsed with sterile phosphate-buffered saline (PBS) containing $0.1 \%(\mathrm{v} / \mathrm{v})$ penicillin-streptomycin $(\mathrm{P} / \mathrm{S})$ solution. Subsequently, the bone marrow was flushed with Roswell Park Memorial Institute 1640 (RPMI 1640, Hyclone) containing 10\% FBS and 0.1\% P/S and filtered with the cell strainer $(70 \mathrm{~mm})$. Cells were resuspended with RPMI 1640 after centrifugation, and then primed with CSF ( $20 \mathrm{ng} / \mathrm{ml}$, PeproTech) with medium exchange every other day for four days to generate $\mathrm{mBMDM}$.

Mouse peritoneal macrophages ( $\mathrm{mPM} \varphi$ ) were isolated from the peritoneal cavities of mice $3 \mathrm{~d}$ after injection with thioglycolate medium and were cultured in DMEM medium supplemented with $10 \%$ FBS. After $2 \mathrm{hr}$ non-adherent cells were removed by thorough washing, and adherent cells ( $\mathrm{mPM} \varphi)$ were infected.

To harvest mouse alveolar macrophages (AMs), broncho-alveolar-lavage was performed. The vein catheter (27G) was installed into the trachea through a small incision after sacrificing the adult mice, and next, PBS with EDTA ( $2 \mathrm{mM}$ ) was administered to unfold the lungs tissue and retrieve the cells in suspension. Cells were centrifuged and seeded into cell culture dishes and stimulated with GM-CSF (20 $\mathrm{ng} / \mathrm{ml}$, PeproTech) for $24 \mathrm{hr}$, and at last, the adherent cells (AMs) were collected for further experiments.

Kupffer cells (KCs) were extracted as described before (Bourgognon et al., 2015; Li et al., 2014a). In brief, adult mice were sacrificed and underwent liver perfusion with Hank's Balanced Salt Solution (HBSS, Hyclone) (from $3 \mathrm{ml} / \mathrm{min}$ to $7 \mathrm{ml} / \mathrm{min}$ ). The excised liver tissues were digested with RPMI 1640 containing $0.1 \%(\mathrm{v} / \mathrm{v})$ type IV collagenase (Sigma-Aldrich) and bathe-watered. Following digestion, the liver homogenate was filtered and centrifuged to acquire the cell suspension. KCs were further separated from hepatocytes and other sinusoidal cells by gradient centrifugation $(300 \mathrm{~g}, 50 \mathrm{~g}$, and $300 \mathrm{~g}$ for 5 min at $4{ }^{\circ} \mathrm{C}$ ), and then purified from satellite cells with the method of selective adherence to plastic. 


\section{Transfection}

The indicated plasmids were transfected into NIH/3T3 cells using JetPEl reagents (Polyplus). siRNA transfection was applied with lipofectamine 2000 (Invitrogen) at $24 \mathrm{~h}$ prior to infection, the sequences of which were shown in Table S4. For LNAs-mediated RNAi, the LNAs were directly added to the medium of mBMDM ( $50 \mathrm{nM}$, the short oligonucleotides would be taken up naturally by cells). The exogenous expression of plasmids in murine macrophages was relied on electrotransfection with the $\mathrm{Neon}^{\mathrm{TM}}$ transfection system instruments (Invitrogen, Cat\# MPK5000). The virus strains used in this paper were preserved in our lab and propagated in Vero E6 cells.

\section{Viral Infection}

Cultured cells were infected with indicated multiplicities of infection (MOI) of HTNV or other viruses. After $2 \mathrm{hr}$, virus-containing medium was discarded and the cells were washed thoroughly with sterile and replaced with the culture medium. As a control, cells were incubated with culture supernatant from uninfected Vero E6 cells, which were referred to as mock-infected cells.

\section{Endothelial Permeability Assessment}

HUVEC were seeded to the middle layer of transwell system for near 5 days to form q compact single-cell stratum (achieving a stable value of transepithelial electrical resistance/ TER as measured with a Millicell ${ }^{\circledR}$ ERS-2 voltohmmeter), and then the PBMC (or monocytes removed PBMC, or human monocytes) and HEK293 cells were seeded at the upper or bottom layer with the ECM, respectively. These cells were co-cultured for $48 \mathrm{hr}$ with gentle shaking and then infected with HTNV (calculating the total cell number of the three layers to compute the virus challenge $\mathrm{MOI}, \mathrm{MOI}=1$ ). For HTNV infection, the upper layer suspension cells were centrifuged and resuspended with HTNV, and then added to the upper layer. After $4 \mathrm{hr}$, the upper medium was replaced with ECM. For blocking the biological effects of TNFa, the neutralizing antibodies $(5 \mu \mathrm{g} / \mathrm{ml})$ were added to the upper chamber. The TER values were measured at indicated points post infection to assess the alteration of endothelial function.

\section{Live Cell Imaging}

The RAW264.7 or THP-1 (primed by PMA) cells stabling expressing GFP-p65 and RFP-ІкB, as well as the WT, RBP-JCKO or Inc-ip65 ${ }^{-/-} \mathrm{mBMDM}$ were seeded into $35 \mathrm{~mm} \mu$-dish (ibidi, Cat\# 81156) and were infected with HTNV $(\mathrm{MOI}=1)$. At the late infection stage, the $\mu$-dish was transferred to the climate chamber $\left(37^{\circ} \mathrm{C}\right.$, $5 \% \mathrm{CO}_{2}$ ) which was connected to the Live Cell Station (A1R-HD25, Nikon). Fluorescent (GFP and RFP filter) images were chosen randomly and acquired with a 40x objective every 10 minutes from $24 \mathrm{hpi}$ to $36 \mathrm{hpi}$. Single images were then merged and movies recorded with the Imaging Software NIS-Elements F Ver4.60.00 (Nikon). At least four visual fields were selected and analyzed for each group, and the presentive view was shown in figures or videos.

\section{Macrophage Function}




\section{Immunophenotype}

To evaluate the in vitro phagocytosis capacity of mBMDM, FAM-labeled RNAs (22 bp, GenePharma) were added to WT or RBP-JCKO mBMDM at 36 hi, or the LNAs-pretreated WT mBMDM at 36 hi (MOI=1, $3 \mu \mathrm{g}$ RNAs/ $2.5 \times 10^{5}$ cells). The FAM ${ }^{+}$macrophages were calculated with the fluorescence microscope at $24 \mathrm{hr}$ post treatment. For assessment of chemotaxis ability of macrophages, the mBMDM and bEnd.3 cells were sed to the middle and bottom layer of the transwell plate $\left(6.5 \mathrm{~mm}\right.$ Transwell ${ }^{\circledR}$ with $5.0 \mu \mathrm{m}$ pore polycarbonate membrane, Corning), and the HTNV was added to the intervals between them at an MOI of 1. The number of migrating macrophages on the back of middle layer (the region towards the bottom) was counted through crystal violet staining at $24 \mathrm{hpi}$. The antigen-presenting ability was measured by the expression of CD80 and CD86 through flow cytometry. The immunoregulation function was detected by the production of cytokines or chemokines with ELISA or qRT-PCR. To assess the anti-microbial ability, cellular ROS production was detected with DCFDA/H2DCFDA. In brief, the mBMDM with indicated treatments were harvested and seeded into a dark, clear bottom 96-well microplate, and stained by incubating with the DCFDA Solution $\left(100 \mu \mathrm{l} /\right.$ well) for $45 \mathrm{~min}$ at $37^{\circ} \mathrm{C}$ in the dark. The plate was measured immediately on a fluorescence plate reader at $\mathrm{Ex} / \mathrm{Em}=485 / 535 \mathrm{~nm}$ in end point mode.

\section{Metabolic Phenotype}

The mitochondrial respiration (oxygen consumption rate, OCR) and glycolysis (extracellular acidification rate, ECAR) of mBMDM were performed. The WT and RBP-J ${ }^{C K O}$ mBMDM, or LNAs-pretreated mBMDM were seeded into a Seahorse XFe96 culture plate (Agilent Technologies) and analyzed at $36 \mathrm{hpi}$ on a Seahorse XFe96 Analyzer (Agilent Technologies). To assess OCR, the oligomycin (1 $\mu \mathrm{M}), \mathrm{FCCP}(0.75 \mu \mathrm{M})$, antimycin $A(1 \mu \mathrm{M})$ and rotenone $(2 \mu \mathrm{M})$ were added at indicated time points. To measure ECAR, Glucose $(10 \mathrm{mM})$, Oligomycin $(1 \mu \mathrm{M})$ and 2-DG $(50 \mathrm{mM})$ were added at indicated time points. The assay protocols were designed and the data were analyzed using Seahorse Wave desktop software (Version: 2.6, Agilent).

\section{Mitochondria Pathophysiology}

The number and morphology change of mitochondria were analyzed with the transmission electron microscopy (TEM) technology. The HTNV-infected WT or RBP-JCKO mBMDM at indicated time points were harvested and fixed with $2.5 \%$ glutaraldehyde on ice for $2 \mathrm{hr}$, which was followed by fixation in $2 \%$ osmium tetroxide. Then the cells were dehydrated with sequential washes in $50 \%, 70 \%, 90 \%, 95 \%$, and $100 \%$ ethanol. Areas containing cells were block mounted and thinly sliced. Sections were photographed using a Hitachi HT7700 transmission electron microscope (Hitachi), and the images were processed with Hitachi TEM system.

\section{RNA-seq, Transcriptomic and LncRNA Data Analysis}

\section{Library Construction and Sequencing}


Total RNA was extracted from WT mBMDM at $0,12,24$ or $36 \mathrm{hpi}$, as well as WT and RBP-JCKO mBMDM at $36 \mathrm{hpi}$, using the TRIzol (Invitrogen). The ribosomal RNA was removed using the Ribo-Zero ${ }^{\text {TM }}$ kit (Epicentre Biotechnologies). Fragmented RNA (the average length was approximately $200 \mathrm{bp}$ ) were subjected to the first strand and second strand CDNA synthesis following by adaptor ligation and enrichment with a low-cycle according to instructions of NEBNext ${ }^{\circledR}$ Ultra ${ }^{\text {TM }}$ RNA Library Prep Kit for Illumina (NEB). The purified library products were evaluated using the Agilent 2200 TapeStation and Qubit ${ }^{\circledR 2.0}$ (Life Technologies). The libraries were paired-end sequenced (PE150, Sequencing reads were 150 bp) at Guangzhou Ribo Biotechnology (Guangzhou, China) using Illumina HiSeq 3000 platform.

\section{Pre-processing of Sequencing Reads \& Quality Control}

To remove trailing sequences below a phred quality score of 20 and achieve uniform sequence lengths for downstream clustering processes, raw fastq sequences were treated with Trimmomatic tools ( $v 0.36)$ using the following options: TRAIL-ING: 20, MINLEN: 235 and CROP: 235. Sequencing read quality was inspected using the FastQC software. Adapter removal and read trimming were performed using Trimmomatic. Sequencing reads were trimmed from the end (base quality less than Q20) and filtered by length (less than 25).

\section{Quantification of Gene Expression Level}

Paired-end reads were aligned to the mouse reference genome mm10 with HISAT2. HTSeq v0. 6.0 was used to count the reads numbers mapped to each gene. The whole samples expression levels were presented as expected number of Reads PerKilobase of transcript sequence per Million base pairs sequenced (RPKM), which is the recommended and most common method to estimate the level of gene expression.

\section{Identification of New LnCRNA}

The raw data were first filtered to remove low-quality reads, then the clean data that passed repeated testing

was assembled using the StringTie based on the reads mapped to the reference genome. The assembled transcripts were annotated using gffcompare program. The unknown transcripts were used to screen for putative IncRNAs. Putative protein-coding RNAs were filtered out using a minimum length and exon number threshold. Transcripts with lengths above $200 \mathrm{nt}$ with predicted ORF shorter than $300 \mathrm{nt}$ were selected as IncRNA candidates. They were subjected to further screening using $\mathrm{CPC} / \mathrm{CNCl} / \mathrm{Pfamto}$ distinguish the protein-coding genes from the noncoding genes.

\section{Differential Expression Analysis}

The statistically significant differential expression genes were obtained by an adjusted P-value threshold of $<0.05$ and $\mid \log 2$ (fold change) | $>1$ using the DEGseq software. Finally, a hierarchical clustering analysis was performed using the $\mathrm{R}$ language package gplots according to the RPKM values of differential genes 
in different groups. And colors represent different clustering information, such as the similar expression pattern in the same group, including similar functions or participating in the same biological process.

\section{GO Terms and KEGG Pathway Enrichment Analysis}

All differentially expressed mRNAs were selected for GO and KEGG pathway analyses. GO was performed with KOBAS 3.0 software. GO provides label classification of gene function and gene product attributes (http://www.geneontology.org). GO analysis covers three domains: cellular component (CC), molecular function (MF) and biological process (BP). The differentially expressed mRNAs and the enrichment of different pathways were mapped using the KEGG pathways with KOBAS 3.0 software.

\section{Molecular Analyses}

\section{Flow Cytometry (FCM)}

Generally, Fcyll/III receptors of macrophages were blocked with anti-CD16/32 antibody (BD Bioscience) before staining the cell surface makers, and the brilliant stain buffer (BD Bioscience) was applied prior to staining intracellular cytokines. For staining the transcription factors (FoxP3, RORyt, GATA3 and T-bet) in T cells from the healthy or patient PBMC, the BD Pharmingen ${ }^{\mathrm{TM}}$ Transcription Factor Buffer Set was applied. The cells were manipulated in the FCM buffer during the flow cytometry assays, which referred to the PBS containing $2 \%$ FBS (Gibco) and 2mM EDTA. For in vitro assays, cells were enzymatically detached with Trypsin-EDTA solution (Solarbio) and subsequently washed and processed with FCM buffer. For the flow cytometry detection of macrophages in spleens, the single cell suspension of the spleen tissue was acquired through gentle grinding and filtration with a $70 \mu \mathrm{m}$ cell strainer, and the erythrocytes were lysed with RBC lysis buffer (Gibco). The main procedure was listed as following: Cell acquisition" Fc receptor block" Surface markers staining" Permeabilization and fixation" brilliant stain buffer treatment" intracellular iNOS or cytokines staining" Compensation adjustment with beads" Samples were analyzed with a 3-laser flow cytometer BD FACSCalibur ${ }^{\text {TM }}$ or 10-laser flow cytometer BD FACSCanto. At last, the data were processed with the FlowJo v10 (TreeStar). Respective antibodies were shown in the Key Resources Table.

\section{Bio-Plex Multiplex Immunoassay}

The serum or cell supernatant samples were centrifuged at $10,000 \mathrm{rpm}$ for $15 \mathrm{~min}$ at $4{ }^{\circ} \mathrm{C}$ and then diluted (for serum, sample diluent HB,1:4; for cell supernatants, culture media, 1:5). After preparing standards, controls and samples, the Bio-plex multiplex immunoassay was conducted as the workflow shown: Prewetting wells" Adding the magnetic beads containing the antibodies against various cytokines and chemokines ( $50 \mu \mathrm{l}$, totally forty kinds of cytokines and chemokines)" Adding the sample/ standard/ control (incubation on shaker at $850 \mathrm{rpm}$ for $1 \mathrm{hr}$ at RT)" Adding biotinylated detection antibodies containing the phycoerythrin fluorescent reporters $(25 \mu \mathrm{l}$, incubation on shaker at $850 \mathrm{rpm}$ for $30 \mathrm{~min}$ at RT)" Adding streptavidin-PE ( $50 \mu \mathrm{l}$, incubation on shaker at $850 \mathrm{rpm}$ for $10 \mathrm{~min}$ at RT)" Resuspending in assay buffer (125 $\mu$ l, shaking at 850 rpm for $30 \mathrm{sec}$ )" Acquiring data on Bio-Plex system (Bio-Rad). 


\section{Enzyme-Linked Immunosorbent Assay (ELISA)}

Sandwich ELISA was applied to detect HTNV NP as we previously described (Ma et al., 2017b). Briefly, the ani-NP mouse monoclonal antibody $1 \mathrm{~A} 8$ was coated on microplates in $0.1 \mathrm{M}$ sodium carbonate bicarbonate buffer ( $\mathrm{pH}$ 9.0) at $4{ }^{\circ} \mathrm{C}$ overnight. The patient serum or mice tissue lysates with RIPA (SigmaAldrich) were collected after centrifuging, and then incubated on the microplates at $37^{\circ} \mathrm{C}$ for $2 \mathrm{hr}$. HRPconjugated $1 \mathrm{~A} 8$ was used as the detection antibody. The absorbance of the color reaction developed using

tetramethylbenzidine (TMB, Abcam) and stop solution $\left(2 \mathrm{M} \mathrm{H}_{2} \mathrm{SO}_{4}\right)$ was measured at $450 \mathrm{~nm}$. An absorbance was required and positive/negative $(P / N)>2.1$ was considered significant. The results were presented with ratios of the sample value versus that of the negative control (P/N value).

Indirect-ELISA was applied to assess the mouse IgG against HTNV NP based on the producer's information (WAITAI BioPharm). In brief, the recombinant NP was coated on microplates and incubated with mice lung tissue lysates (dilution with 1:30 by PBS). HRP-conjugated anti-mouse IgG antibody was added and the absorbance was assessed post TMB treatment at $450 \mathrm{~nm}$. The results were shown as antiNP IgG positive or negative to analyze the disease phase stage for the HTNV-infected field mice.

The concentration of multiple cytokines and chemokines from cell supernatants or mice tissues was evaluated with ELISA kits (Abcam or R\&D Systems) according to the manufacturer's instructions. In short, standard samples were prepared with gradient dilution to build the standard curve. The samples were diluted with special buffer and added to the plated pre-coated with the anti-cytokine or chemokine antibodies and then reacted with HRP-conjugated detection antibodies. TMB and stop solution were added in sequential to measure the OD450. The cytokine or chemokine concentration was calculated with the standard curve.

\section{Protein Preparation}

For to detect the M1/M2-related signaling in macrophages or confirm the overexpression efficacy in co-IP experiments, the whole cell lysates (WCL) were collected with the RIPA lysis buffer (Sigma-Aldrich) supplemented with protease and phosphatase inhibitors (Sigma-Aldrich) for further immunoblot analysis. To assess the activation of NF-KB, JAK/STAT or IRF pathway post HTNV infection in murine versus human macrophages, the translocation of key transcription factors, such as p65, Stat1, IRF4 and IRF5, was determined with the nuclear and cytoplasmic extraction reagents (Thermo Fisher). In brief, the human or murine macrophages were harvested at indicated time points with trypsin-EDTA (Solarbio). The cell pellets were acquired through washing and centrifuging. Then, CER I $(100 \mu \mathrm{l})$ was added to the packed cells $(10 \mu \mathrm{l})$ with vigorous vortex for $15 \mathrm{sec}$ to fully suspend the cell pellet and incubation on ice for 10 min, destroying the cell membrane but not karyolemma. Next, ice-cold CER II (5.5 $\mu$ l) was added to the sample with vigorous vortex for $5 \mathrm{sec}$, incubation on ice for $1 \mathrm{~min}$, and repeated vigorous vortex for 5 sec. Supernatants containing the cytoplasmic extracts were collected after centrifuging at $16,000 \mathrm{~g}$ for 10 min. The insoluble pellets were suspended in ice-cold NER $(50 \mu l)$ with intermittent vigorous vortex for 15 
$\mathrm{sec}$ and incubation on ice for $10 \mathrm{~min}$, this procedure of which was repeated for 4 times (totally near 40 min). Finally, the supernatant fraction containing nuclear extracts was obtained after centrifuging at $16,000 \mathrm{~g}$ for $15 \mathrm{~min}$.

\section{Immunoblot Assay}

The protein concentration was first determined based on Bicinchoninic acid (BCA) method using the Compat-Able ${ }^{\mathrm{TM}}$ BCA Protein Assay Kit (Thermo Fisher). Equal amounts of protein were boiled at $95^{\circ} \mathrm{C}$ for 10 min, separated by SDS-PAGE at different concentrations, and then electrophoretically transferred onto polyvinylidene fluoride membranes (PVDF). After blocking with 5\% non-fat milk in TBS, the membrane was incubated with the primary antibodies, followed by secondary antibodies labeled with infrared dyes. For the assessment of protein phosphorylation, the antibody targeted at various phosphorylated points were applied separately or combinedly for the first scanning, and then the PVDF membrane was striped with the restore buffer (Thermo Fisher) and underwent secondary antibody incubation with for the total proteins, as well as the infrared dye-labeled antibodies. The signals on the PVDF membrane were visualized using the Odyssey Infrared Imaging System (LI-COR Biosciences).

\section{Co-immunoprecipitation (Co-IP) Assay}

Cells transfected with the appropriate plasmids were harvested and lysed with IP-lysis buffer (50 mM Tris$\mathrm{HCl}$ [pH 7.4], $150 \mathrm{mM} \mathrm{NaCl}, 1 \%$ [w/v] Triton X-100, $1 \mathrm{mM}$ EDTA [pH 8.0], 0.1\% [v/v] SDS, and protease inhibitor cocktail) for $30 \mathrm{~min}$. The supernatants were collected via centrifugation at 13,000 rpm for 25 min at $4{ }^{\circ} \mathrm{C}$. The protein extract was incubated with the equilibrated magnetic beads (for assessing the protein interaction with the exogenous expressing system; beads of Bimake), or protein $\mathrm{G}$ sepharose (for detecting the endogenous interaction; sepharose of Proteintech) that have been co-incubated with desired primary antibodies, overnight at $4{ }^{\circ} \mathrm{C}$. Beads or sepharose were collected and washed three times with washing buffer ( $5 \%$ [w/v] sucrose, $5 \mathrm{mM}$ Tris-HCl [pH 7.4], $5 \mathrm{mM}$ EDTA [pH 8.0], $500 \mathrm{mM} \mathrm{NaCl}, 1 \%$ [v/v] Triton $\mathrm{X}-100$ ). Then, the beads were boiled at $100^{\circ} \mathrm{C}$ for $5 \mathrm{~min}$ in $5 \times$ SDS protein loading buffer and analyzed by immunoblot.

\section{Immunofluorescence Assay (IFA)}

Cells with indicated treatment were fixed with ice-cold $4 \%(\mathrm{w} / \mathrm{v})$ paraformaldehyde (PFA, Sigma-Aldrich) for $15 \mathrm{~min}$ and then were permeabilized with $0.1 \%$ Triton X-100 (Sigma-Aldrich) for 20 min at RT. After blocking with $3 \%$ bovine serum albumin (BSA, Sigma-Aldrich) for 30 min., the specific primary antibodies (1:50 to 1:200 dilution) were added and incubated overnight at $4^{\circ} \mathrm{C}$. After five washes with DPBS, the secondary antibodies, namely FITC-, Cy3- or Cy5-conjugated goat anti-rabbit or goat anti-mouse IgG (Abcam) was used for detection (incubation at $37^{\circ} \mathrm{C}$ for $1 \mathrm{hr}$ ). Cell nuclei were stained with DAPI (Thermo Fisher) for 5 min at RT. After sealing with the ProLong ${ }^{\text {TM }}$ Gold Antifade Mountant (Thermo Fisher), the samples were observed using a fluorescence microscope (A1R-HD25, Nikon). To observe the localization relationship between IncRNAs and p65, the IFA was performed post the FISH or RNAScope experiments. 
Total cellular RNA was extracted with the TRIzol reagent (Invitrogen) and the Total RNA Extraction Kit (TIANGEN Biotech), the concentration of which was measured with a spectrophotometer. Quantitative real-time PCR (qRT-PCR) was performed with PrimeScript RT Master Mix (TaKaRa) according to the manufacturer's protocol. Each cDNA sample was denatured at $95^{\circ} \mathrm{C}$ for 5 min and amplified for 35 cycles of procedures including $15 \mathrm{~s}$ at $98^{\circ} \mathrm{C}, 30 \mathrm{~s}$ at $58^{\circ} \mathrm{C}$, and $30 \mathrm{~s}$ at $72^{\circ} \mathrm{C}$ with the LightCycler 96 (Roche). The mRNA expression level of each target gene was normalized to the respective GAPDH and analyzed using the LightCycler ${ }^{\circledR} 96$ Application Software (Roche). The qRT-PCR primers were listed in Table S3. To note, five pairs of qRT-PCR primers for the newly identified IncRNAs by RNA-seq (Figure 5B) were designed and applied. The suitable primers were screened with stable results of three independent experiments and listed in Table S3. Northern blot was performed using NorthernMax Kit (Ambion) with biotin labeled probes, the sequences of which were shown in Table S4.

\section{Fluorescence in situ hybridization (FISH) And RNAScope Assays}

FISH was performed with a FISH kit (Ribo Biotechnology) according to the manufacturer's instructions. In brief, cells were fixed with 4\% PFA (Sigma-Aldrich) for 10 min at RT and permeabilized with $0.5 \%$ Triton X100 (Sigma-Aldrich) for 15 min at RT. Prehybridization was performed with IncRNA FISH probe mix at $37^{\circ} \mathrm{C}$ for $30 \mathrm{~min}$, and then hybridization was performed by adding IncRNA FISH probe mix and incubating the mixture at $37^{\circ} \mathrm{C}$ overnight. After washing with $4 \times, 2 \times$, and $1 \times \mathrm{SSC}(1 \times \mathrm{SSC}$ is $0.15 \mathrm{M} \mathrm{NaCl}, 0.015 \mathrm{M} \mathrm{Na}$ citrate), the cell nuclei were stained with DAPI (Thermo Fisher). RNAScope was performed with RNAscope Fluorescent Multiplex Reagent Kit (ACD Bio) based on the manufacturer's protocols. In short, cells were firstly placed on slides and fixed in 4\% PFA (Sigma-Aldrich) for $30 \mathrm{~min}$, followed by antigen repair with RNAscope ${ }^{\circledR}$ hydrogen peroxide (ACD Bio) for 10 min at RT and digestion with RNAscope ${ }^{\circledR}$ protease III (ACD Bio) for another 10 min at RT in the humidifying box. Next, cells were then incubated in order at $40^{\circ} \mathrm{C}$ with the following solutions: (1) RNAScope probes of target RNAs, namely Inc-ip65-C3 and HTNV-S$\mathrm{C} 2$ (v/v, 1:1), in hybridization buffer A (6×SSC, $25 \%$ formamide, $0.2 \%$ lithium dodecyl sulfate, blocking reagents), for $2 \mathrm{hr}$; (2) preamplifier (AMP1, $2 \mathrm{nM})$ in hybridization buffer $B(20 \%$ formamide, $5 \times S S C, 0.3 \%$ lithium dodecyl sulfate, $10 \%$ dextran sulfate, blocking reagents) for 30 minutes; (3) amplifier (AMP2, 2 $\mathrm{nM}$ ) in hybridization buffer $\mathrm{B}$ at $40^{\circ} \mathrm{C}$ for 30 minutes; (4) label probe (AMP3, $2 \mathrm{nM}$ ) in hybridization buffer C ( $5 \times$ SSC, $0.3 \%$ lithium dodecylsulfate, blocking reagents) for 15 minutes. After each hybridization step, slides were washed with wash buffer $(0.1 \times S S C, 0.03 \%$ lithium dodecyl sulfate) three times at RT. Then, the probe signaling was further recognized and amplified by HRP-C2 (ACD Bio) (for 15 min at $40^{\circ} \mathrm{C}$ ), followed by chromogenic detection with TSA ${ }^{\circledR}$ Plus Cy3(Akoya Biosciences) (for 30 min at $40^{\circ} \mathrm{C}$ ) for detecting HTNV-S. After treatment with HRP-C2-blocker (ACD Bio), foresaid steps were repeated with HRPC3 (ACD Bio) and TSA® Plus Cy5 (Akoya Biosciences) for assessing Inc-ip65. Finally, after the DAPI staining and Prolong Gold Antifade Mountant (Thermo Fisher) treatment, the samples underwent IFA for p65 detection, or directly observed with the confocal microscope (Nikon).

\section{RNA immunoprecipitation (RIP) Assay}


RNA immunoprecipitation was performed using Magna RIP ${ }^{\text {TM }}$ RNA-Binding Protein Immunoprecipitation Kit (Millipore) according to manufacturer's instructions at RNase-free environment. Briefly, the mBMDM or RAW264.7 cells that were electrotransfected with indicated proteins and IncRNAs for $48 \mathrm{hr}$, or mBMDM at different time points post HTNV infection, were collected and treated with RIP Lysis Buffer. The anti-myc antibody conjugated magnetic beads (targeting myc-p65 or related mutants), or primary antibodies enriched by protein $A+G$ magnetic beads (targeting M1- or M2-relate transcription factors or NF-KB pathway components) were incubated with cell lysates on shaker overnight at $4{ }^{\circ} \mathrm{C}$. For the positive control, the anti-SNRNP70 antibody that could pull down the U1 snRNA was applied. The supernatants were discarded after washing on the magnetic frame, and then sediments were added with proteinase $\mathrm{K}$ with gentle shaking for $30 \mathrm{~min}$ at $55^{\circ} \mathrm{C}$. At last, the supernatants were collected on the magnetic frame, from which the total target protein attached RNAs were extracted as above-mentioned. The enriched IncRNAs were detected by qRT-PCR, normalized to the positive control (U1 snRNA).

\section{Dual-Luciferase Reporter Assay}

RAW264.7 or THP-1 cells were co-transfected with pNF-KB-luc, pRL-TK and indicated plasmids. Cells in 24-well plates were infected with HTNV (MOI=1) $36 \mathrm{hr}$ after electrotransfection, and then harvested and lysed. The luciferase activity was measured using the Dual-Glo Luciferase Assay System (Promega) according to the manufacturer's instructions. Luciferase activity was normalized to Renilla luciferase activity.

\section{Tissue Analyses}

\section{Histological Analyses}

Paraffin embedded tissue samples were sectioned and stained with hematoxylin and eosin for histomorphological analysis. First, deparaffinize and hydrate to water, and process slides as following sequentially: Xylene I for 20 min, Xylene II for 20 min; 100\% alcohol I for 5 min; 100\% alcohol II for 5 min; $75 \%$ alcohol for $5 \mathrm{~min}$; and then rinse in water. Second, stain in hematoxylin solution, that is to immerse slides in hematoxylin solution for 3 to $5 \mathrm{~min}$, and rinse them in water. Then differentiate sections with acid alcohol, rinse again. Blue up sections with ammonia solution, wash in slowly running tap water. Third, process slides as following sequentially for eosin staining: $85 \%$ alcohol I for $5 \mathrm{~min}, 95 \%$ alcohol II for $5 \mathrm{~min}$ and eosin for $5 \mathrm{~min}$. Finally, dehydrate and mount as following sequentially: $100 \%$ alcohol I for 5 min, $100 \%$ alcohol II for $5 \mathrm{~min}, 100 \%$ alcohol III for $5 \mathrm{~min}$, Xylene I for $5 \mathrm{~min}$, Xylene II for $5 \mathrm{~min}$ and mounted with resin. Slides were scanned with the Panoramic MIDI (3DHISTECH). Immunostaining of paraffin sections was preceded by different antigen unmasking methods. Immunohistochemical staining was performed on paraffin-embedded tissue sections, using anti-HTNV NP antibodies (1A8 prepared by our lab) and related secondary antibodies, followed by chromogenic detection with DAB.

\section{Tissue TUNEL and IFA}


Tissue TUNEL assays were performed with the TUNEL Assay Kit (Enhanced FITC) (Elabscience) bases on the manufacturer's instructions. In short, the freezing section samples of different mice tissues were fixed with 4\% PFA, followed by incubation with Terminal Deoxynucleotidyl Transferase (TdT) Equilibration working buffer at RT for 30 min and TdT Enzyme working solution at $37^{\circ} \mathrm{C}$ for $30 \mathrm{~min}$ in a wet bow. Then nuclei were stained with DAPI and the slides were sealed with the mounting medium. The tissue IFA was based on the frozen sections, the procedure of which was similar to cellular IFA. The imaging data were acquired with the Panoramic MIDI (3DHISTECH).

\section{QUANTIFICATION AND STATISTICAL ANALYSIS}

Statistical analysis was performed with GraphPad Prism (GraphPad software, Version 8). For comparison of two groups, unpaired two-tailed unpaired Student's $t$ test was applied unless stated otherwise in the figure legend. For multiple comparisons, one- or two-way ANOVA were performed, followed by Tukey's multiple comparison tests. Survival analysis was performed with log-Rank [Mantel-Cox] test. Differences

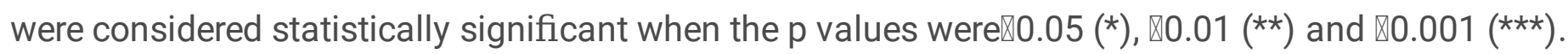
Statistically non-significant data ( $p$ value $>0.05$ ) are indicated as NS. Data are presented as mean \pm SEM if not stated otherwise in the figure legend. The number of mice and the number of independent experiments conducted is shown in the figure legend.

\section{KEY RESOURCES TABLE}




\section{Antibodies}

\section{Flow Cytometry Assays}

Alexa Fluor ${ }^{\circledR} 488$ Rat Anti- $\quad$ BD Biosciences Mouse IL-6 (MP5-20F3)

Alexa Fluor ${ }^{\circledR} 647$ Rat AntiMouse CD14 (rmC5-3)

Alexa Fluor ${ }^{\circledR} 647$ Rat AntiMouse CD206 (MR5D3)

APC-Cy ${ }^{\text {TM }} 7$ Mouse Anti-Human BD Biosciences CD16 (3G8)

APC-Cy ${ }^{\text {TM }} 7$ Mouse Anti-Human BD Biosciences CD3 (SK7)

APC-Cy ${ }^{\text {TM }} 7$ Rat Anti-CD11b BD Biosciences (M1/70)

APC-R700 Mouse Anti-Human BD Biosciences IL-17A (N49-653)

BB515 Mouse Anti-Human CD 4 (RPA-T4)

BB700 Hamster Anti-Mouse BD Biosciences CD11C (HL3)

BB700 Rat Anti-Mouse CD197 BD Biosciences (CCR7) (4B12)

BB700 Rat Anti-Mouse TNF BD Biosciences (MP6-XT22)

BUV661 Mouse Anti- Human BD Biosciences HLA-DR (G46-6)

BV421 Mouse Anti-Human RORyt (Q21-559)

BV421 Rat Anti-Human and Viral IL-10 (JES3-9D7)

BV421 Rat Anti-Human and BD Biosciences Viral IL-10 (JES3-9D7)

BV421 Rat Anti-Mouse CX3CR1 (Z8-50)

BV480 Rat Anti-Mouse F4/80 BD Biosciences (T45-2342)

BV510 Mouse Anti-Human
BD Biosciences

BD Biosciences

BD Biosciences

BD Biosciences
Cat\# 561363; RRID:

AB_10694253

Cat\# 565743; RRID:

AB_2739340

Cat\# 565250; RRID:

AB_2739133

Cat\# 557758; RRID:

AB_396864

Cat\# 557832; RRID:

AB_396890

Cat\# 557657; RRID:

AB_396772

Cat\# 565163; RRID:

AB_2739087

Cat\# 564419; RRID:

AB_2744419

Cat\# 566505; RRID:

AB_2869773

Cat\# 566462; RRID:

AB_2744307

Cat\# 566511; RRID:

AB_2869775

Cat\# 612980

Cat\# 563282; RRID:

AB_2738114

Cat\# 564053; RRID:

AB_2738566

Cat\# 564053; RRID:

AB_2738566

Cat\# 567531

Cat\# 565635; RRID:

AB_2739313

Cat\# 563079; RRID: 
CD14 (M $\varphi \mathrm{P9})$

BV510 Mouse Anti-Human

IFN-y (B27)

BV605 Mouse Anti-Human

CD206 (19.2)

BV605 Mouse Anti-Human

CD25 (2A3)

BV605 Rat Anti-Mouse CD192

(CCR2) (475301)

BV650 Mouse Anti-Human

CD11c (B-ly6)

FITC Mouse Anti-HTNV NP

(1A8)

FITC Rat Anti-Mouse IL-10

(JES5-16E3)

FITC Rat Anti-Mouse Ly-6C BD Biosciences (AL-21)

FITC Rat Anti-Mouse TNF (MP6-XT22)

FITC Mouse Anti-Human

CD11b (ICRF44)

PE Hamster Anti-Mouse CD80 BD Biosciences (16-10A1)

PE Mouse anti-Human FoxP3 BD Biosciences (236A/E7)

PE Mouse Anti-Human IL-8

(G265-8)

PE Rat Anti-Mouse CD86

(GL1)

PE Rat Anti-Mouse F4/80

(T45-2342)

PE Rat Anti-Mouse IL-12

(p40/p70) (C15.6)

PE Rat Anti-mouse iNOS

(CXNFT)

PE-Cy ${ }^{\text {TM }} 7$ Mouse Anti-GATA3 BD Biosciences

(L50-823)

PerCP-Cy ${ }^{\text {TM }}$ 5.5 Mouse Anti-

Human TNF (MAb11)

2002)

BD Biosciences
AB_2737993

Cat\# 563287; RRID:

AB_2738118

Cat\# 740417; RRID:

AB_2740147

Cat\# 562660; RRID:

AB_2744343

Cat\# 747969; RRID:

AB_2872430

Cat\# 563404; RRID:

AB_2732048

$\mathrm{N} / \mathrm{A}$

Cat\# 554466; RRID:

AB_395411

Cat\# 561085; RRID:

AB_394628

Cat\# 561064; RRID:

AB_395379

Cat\# 562793; RRID:

AB_1645544

Cat\# 561955; RRID:

AB_395039

Cat\# 560852; RRID:

AB_10563418

Cat\# 554720; RRID:

AB_395529

Cat\# 561963; RRID:

AB_10896971

Cat\# 565410; RRID:

AB_2687527

Cat\# 554479; RRID:

AB_395420

Cat\# 12-5920-82; RRID:

AB_2572642

Cat\# 560405; RRID:

AB_1645544

Cat\# 560679; RRID:

AB_1727579 
PerCP-Cy $\mathrm{T}^{\mathrm{TM}}$ 5.5 Mouse Anti-T- $\quad$ BD Biosciences bet (04-46)
Cat\# 561316; RRID:

AB_10611726

\section{Immunoblot \& Immunofluorescent Measurements}

\begin{tabular}{|c|c|c|}
\hline Anti-NF-kB p65 Antibody & Abcam & $\begin{array}{l}\text { Cat\# ab16502; RRID: } \\
\text { AB_443394 }\end{array}$ \\
\hline $\begin{array}{l}\text { Anti-activated Notch1 } \\
\text { Antibody (NICD) }\end{array}$ & Abcam & $\begin{array}{l}\text { Cat\# ab8925; RRID: } \\
\text { AB_306863 }\end{array}$ \\
\hline Anti-CD34 Antibody [EP373Y] & Abcam & $\begin{array}{l}\text { Cat\# ab81289; RRID: } \\
\text { AB_1640331 }\end{array}$ \\
\hline $\begin{array}{l}\text { Anti-DDDDK Tag (Binds to } \\
\text { FLAG® tag sequence) } \\
\text { Antibody [F-tag-01] }\end{array}$ & Abcam & $\begin{array}{l}\text { Cat\# ab18230; RRID: } \\
\text { AB_444336 }\end{array}$ \\
\hline
\end{tabular}

Anti-ERK1+ERK2 (phospho

Abcam

Cat\# ab278538

T202 + Y204) Antibody

[ERK12T202Y204-A11]

\section{Anti-ERK1+ERK2 Antibody [EPR17526]}

Anti-F4/80 Antibody [Cl: A3- Abcam

1]

Anti-GAPDH Antibody [6C5] Abcam

Anti-GFP Antibody

Anti-HA Tag Antibody

Anti-IKKa+IKKß (phospho

S180+S181) Antibody

Anti-IKKa+IKKß Antibody

[EPR16628]

Anti-iNOS Antibody

[EPR16635]

Anti-IRF4 Antibody

Anti-IRF5 Antibody

[EPR17067]

Anti-IKB a (phosphoS36)

Antibody [EPR6235(2)]

Anti-IKBa (phospho S32)

Antibody [EPR3148]

Anti-IKBa Antibody [E130]
Abcam

Abcam

Abcam

Abcam

Abcam

Abcam

Santa Cruz Biotechnology

Abcam

Abcam

Abcam

Abcam
Cat\# ab184699; RRID:

AB_2802136

Cat\# ab6640; RRID:

AB_1140040

Cat\# ab8245; RRID:

AB_2107448

Cat\# ab290; RRID: AB_303395

Cat\# ab9110; RRID:

AB_307019

Cat\# ab55341; RRID:

AB_883038

Cat\# ab178870

Cat\# ab210823; RRID:

AB_2861417

Cat\# sc-48338; RRID:

AB_627828

Cat\# ab181553; RRID:

AB_2801301

Cat\# ab133462; RRID:

AB_2801653

Cat\# ab92700; RRID:

AB_10562951

Cat\# ab32518; RRID:

AB_733068 
Anti-Jagged1 Antibody

Anti-Jagged2 Antibody [EPR3646]

Anti-JNK1 (phospho T183/Y185) Antibody [EPR20763]

Anti-JNK1 Antibody [EPR17557]

Anti-Lamin B1 Antibody

Anti-Myc Tag Antibody [9E10]

Anti-NF-kB p65 (phospho

S276) Antibody

Anti-NF-kB p65 (phospho Abcam

S468) Antibody

Anti-NF-kB p65 (phospho S529) Antibody

Anti-NF-kB p65 (phospho S536) Antibody

Anti-Notch1 Antibody [EP1238Y]

Anti-Notch2 Antibody

Anti-Notch3 Antibody

Anti-STAT1 (phospho S727) Abcam

Antibody [EPR3146]

Anti-STAT1 (phospho Y701) Abcam

Antibody

Anti-STAT1 Antibody

Abcam

Anti-STAT3 (phospho S727)

Antibody [E121-31]

Anti-STAT3 (phospho Y705)

Antibody [EPR23968-52]

Anti-STAT3 Antibody

[EPR787Y]

Anti-Tubulin Antibody

Abcam

Abcam

Abcam

Abcam

Abcam

Abcam

Abcam

Abcam

Abcam

Abcam

Abcam

Abcam

Abcam

Abcam

Abcam

Abcam
Cat\# ab199380

Cat\# ab194726

Cat\# ab16048; RRID:

AB_443298

Cat\# ab32; RRID: AB_303599

Cat\# ab31473; RRID:

AB_881299

Cat\# ab97726; RRID:

AB_10681170

Cat\# ab86299; RRID:

AB_1925243

Cat\# ab52627; RRID:

AB_881725

Cat\# ab137665

Cat\# ab23426; RRID:

AB_776841

Cat\# ab109461; RRID:

AB_10863745

Cat\# ab30645; RRID:

AB_779082

Cat\# ab47425; RRID:

AB_882708

Cat\# ab32143; RRID:

AB_2286742

Cat\# ab267373

Cat\# ab68153; RRID:

AB_2889877
Cat\# ab6046; RRID: 
AB_2210370

Donkey Anti-Goat lgG H\&L Abcam (Cy3 $\AA$ )

FITC Anti-NF-kB p65 (phospho S536) Antibody

[NFKBp65S536-B7]

Goat Anti-Mouse IgG H\&L (Cy3 $\AA$ )

Goat Anti-Mouse IgG H\&L (Cy5 $\AA)$

Goat Anti-Rabbit IgG H\&L

(Cy3 $\AA$ )

Goat Anti-Rabbit IgG H\&L

(Cy5 $\AA)$

Human/Mouse/Rat RelA/NF R\&D Systems KB p65 Antibody

IRDye ${ }^{\circledR}$ 680RD Goat AntiMouse $\lg G(H+L)$

IRDye ${ }^{8} 800 \mathrm{CW}$ Goat AntiRabbit IgG $(\mathrm{H}+\mathrm{L})$

Mouse monoclonal AntiHTNV Gn (Gn-1)

Mouse monoclonal AntiHTNV NP (1A8)

Mouse/Rat Notch1 Antibody

Phospho-NF-kB p65/RelA-

S276 Rabbit pAb

Phospho-NF-kB p65/RelA-

S468 Rabbit pAb

Phospho-NF-kB p65/RelAS529 Rabbit pAb

Phospho-NF-kB p65/RelAS536 Rabbit pAb

Rabbit Anti-Rat IgG H\&L (FITC)

Abcam

Abcam

Abcam

Abcam

Abcam

LI-COR

LI-COR 2002) 2002)

R\&D Systems

ABclonal

ABclonal

ABclonal

ABclonal

Abcam
Prepared by our Lab (Xu et al.,

Prepared by our Lab (Xu et al.,

\section{Neutralizing \& ELISA Experiments}

Anti-TNF alpha Antibody [2C8]
Cat\# ab6949; RRID:

AB_955018

Cat\# ab278631

Cat\# ab97035; RRID:

AB_10680176

Cat\# ab6563; RRID:

AB_955068

Cat\# ab6939; RRID:

AB_955021

Cat\# ab6564; RRID:

AB_955061

Cat\# AF5078; RRID:

AB_2179033

Cat\# 925-68070; RRID:

AB_2651128

Cat \#926-32211; RRID:

AB_621843

N/A

N/A

Cat\# AF1057; RRID:

AB_2153372

Cat\# AP0123; RRID:

AB_2771505

Cat\# AP0446; RRID:

AB_2771508

Cat\# AP0944; RRID:

AB_2863855

Cat\# AP0475; RRID:

AB_2771511

Cat\# ab6730; RRID:

AB_955327
Abcam

Cat\# ab8348; RRID:

AB_306503 
Anti-Flavivirus Group Antigen Absolute Antibody

[D1-4G2-4-15 (4G2)]

HRP-labeled 1A8 for NP

Detection by ELISA

Mouse Monoclonal Anti-

HTNV GP (3D8)

TNF-alpha/TNFA/TNFSF2

Neutralizing Antibody

\section{Virus Strains}

Prepared by our Lab (Ma et al., 2017b)

Prepared by our Lab (Xu et al., 2002)

SinoBiological
Cat\# Ab00230-2.0; RRID:

AB_2715504

$\mathrm{N} / \mathrm{A}$

N/A

Cat\# 50349-RN023
Hantaan Virus (HTNV, 76118)

Dengue Virus 2 (DENV2)

DH5a

Enterovirus 71 (EV71)
Conserved in our lab (Ma et al., N/A 2017)

Conserved in our lab (Han et al., N/A 2017)

TransGen Biotech

Conserved in our lab (Ye et al., 2020)

Herpes Simplex Type 2 (HSV- Conserved in our lab

2)

Sendai Virus (SeV)
Vesicular Stomatitis Virus
(VSV)
Critical Commercial Assays

Bio-Plex Calibration Kit

Bio-Rad

Cat\# 171203060

Bio-Plex Human 40-plex

Bio-Rad

Bio-Rad

Cat\# 171AK99MR2

Bio-Plex Validation Kit

Abcam

Cat\# 171203001

BrdU Immunohistochemistry

Kit

Co-immunoprecipitation Kit

Compat-Able ${ }^{\top M}$ BCA Protein

Assay Kit

DCFDA/H2DCFDA - Cellular

ROS Assay Kit

Diagnostic Kit for IgG

Antibody to Hantaviruses

(ELSIA)

Proteintech

Thermo Fisher

Abcam

WAITAI BioPharm

Cat\# YZB/Guo 3760-2014
Dual-Luciferase Assay Kit

EasySep ${ }^{\text {TM }}$ Human Monocyte
Promega

StemCell
Cat\# ab113851

Cat\# ab125306

Cat\# KIP-1

Cat\# 21063

Cat\# TM040

Cat\# 19359 
Isolation Kit

Fixation/Permeablization Kit

Fluorescent In Situ

Hybridization Kit

H\&E staining kit

Human Interferon alpha 1

ELISA Kit

Human TNF alpha ELISA Kit

Magna RIP ${ }^{\mathrm{TM}}$

Mouse IL-1 beta/IL-1F2

Quantikine ELISA Kit

Mouse IL-10 ELISA Kit

Mouse IL-6 Quantikine ELISA

Kit

Mouse Interferon alpha 1

ELISA Kit

Mouse IP-10 ELISA Kit

(CXCL10)

Mouse MCP1 ELISA Kit

(CCL2)

Mouse TNF alpha ELISA Kit

Ms Ig Kpa Comp Bead Set

Neon $^{\mathrm{TM}}$ Transfection System

Kit

NE-PER Nuclear and

Cytoplasmic Extraction

Reagents

NF-kB p65 Transcription

Factor Assay Kit

NorthernMax ${ }^{\text {TM }}$ Kit

PrimeScript $^{\mathrm{TM}} \mathrm{RT}$ Reagent Kit

(Perfect Real Time)

Rat Ig Kpa Comp Bead Set

RNA-Binding Protein

Immunoprecipitation Kit

RNAscope 3-plex Negative Control Probes
BD Biosciences

Cat\# 554714

Ribo Biotechnology

Cat\# C10910

Servicebio

Cat\# G1005

Abcam

Cat\# ab213479

Abcam

Cat\# ab181421

Millipore Sigma

Cat\# 17-700

R\&D Systems

Cat\# MLB00C

Abcam

Cat\# ab108870

R\&D Systems

Cat\# M6000B

Abcam

Cat\# ab252352

Abcam

Cat\# ab214563

Abcam

Cat\# ab208979

Abcam

Cat\# ab208348

BD Biosciences

Cat\# 552843

Invitrogen

Cat\# MPK10096

Thermo Fisher

Cat\# 78833

Abcam

Cat\# ab133112

Invitrogen

Cat\# AM1940

TaKaRa

Cat\# RR037B

BD Biosciences

Cat\# 552844 


\begin{tabular}{lll}
$\begin{array}{l}\text { RNAscope Fluorescent } \\
\text { Multiplex Reagent Kit }\end{array}$ & ACD Bio & Cat\# 320850 \\
\hline RNAscope Probe Diluent & ACD Bio & Cat\# 300041 \\
\hline SDS-PAGE Gel kit & CW Bio & Cat\# CW0022S \\
$\begin{array}{l}\text { SYBR Premix EX Taq } \\
\text { (Perfect Real Time) }\end{array}$ & TaKaRa & Cat\# RR420A \\
\hline $\begin{array}{l}\text { Total RNA Extraction Kit } \\
\begin{array}{l}\text { Transcription Factor Buffer } \\
\text { Set }\end{array}\end{array}$ & TIANGEN Biotech & Cat\# DP419 \\
\hline $\begin{array}{l}\text { Transcription Factor Buffer } \\
\text { Set }\end{array}$ & BD Biosciences & Cat\# 562574 \\
\hline
\end{tabular}

\section{Chemicals, Peptides, and Recombinant Proteins}

\begin{tabular}{|c|c|c|}
\hline 2-Deoxy-D-glucose (2-DG) & Selleck & Cat\# S4701 \\
\hline $\begin{array}{l}\text { 4',6-diamidino-2-phenylindole } \\
\text { (DAPI) }\end{array}$ & Thermo Fisher & Cat\# D9542 \\
\hline Alcohol & Sinopharm & Cat\# 100092683 \\
\hline Ammonia solution & Servicebio & Cat\# G1005-4 \\
\hline Anti-Flag magnetic beads & Bimake & Cat\# B26102 \\
\hline Anti-HA magnetic beads & Bimake & Cat\# B26202 \\
\hline Anti-Myc magnetic beads & Bimake & Cat\# B26302 \\
\hline Antimycin A & Sigma-Aldrich & Cat\# A8674-25MG \\
\hline Bovine Serum Albumin (BSA) & Sigma-Aldrich & Cat\# A1933 \\
\hline Brilliant Stain Buffer & BD Biosciences & Cat\# 563794 \\
\hline $\begin{array}{l}\text { Carbonyl cyanide 4- } \\
\text { (trifluoromethoxy) } \\
\text { phenylhydrazone (FCCP) }\end{array}$ & Sigma-Aldrich & Cat\# C2920-10MG \\
\hline $\begin{array}{l}\text { Clophosome }{ }^{\circledR}, \text { Clodronate } \\
\text { Liposomes (Neutral) }\end{array}$ & FormuMax & Cat\# F70101C-N-10 \\
\hline $\begin{array}{l}\text { cOmplete }{ }^{\mathrm{TM}} \text {, Mini Protease } \\
\text { Inhibitor Cocktail }\end{array}$ & Sigma-Aldrich & Cat\# 4693124001 \\
\hline $\begin{array}{l}\text { Antisense LNA }{ }^{\mathrm{TM}} \text { GapmeR } \\
\text { Standard for IncRNA MSTRG- } \\
22387.1\end{array}$ & QIAGEN & $\begin{array}{l}\text { Cat\# } 339511 \text { LG00239397- } \\
\text { DDA }\end{array}$ \\
\hline $\begin{array}{l}\text { Antisense LNA }{ }^{T M} \text { GapmeR } \\
\text { Standard for IncRNA MSTRG- }\end{array}$ & QIAGEN & $\begin{array}{l}\text { Cat\# } 339511 \text { LG00239366- } \\
\text { DDA }\end{array}$ \\
\hline
\end{tabular}


Antisense LNA ${ }^{T M}$ GapmeR QIAGEN

Standard for IncRNA MSTRG-

30740.1-2

Antisense LNA ${ }^{T M}$ GapmeR

Standard for IncRNA MSTRG-

30740.1-3

Antisense LNA ${ }^{\text {TM }}$ GapmeR

QIAGEN

Standard for IncRNA MSTRG-

30740.1-4

Antisense LNA ${ }^{\mathrm{TM}}$ GapmeR

QIAGEN

Cat\# 339511 LG0023975-DDA

Standard for IncRNA MSTRG-

30740.1-10

Antisense LNA ${ }^{\text {TM }}$ GapmeR

Standard for IncRNA MSTRG-

30928.1-1

Antisense LNA ${ }^{\mathrm{TM}}$ GapmeR

Standard for IncRNA MSTRG-

30928.1-2

Custom LNA ${ }^{\mathrm{TM}}$ Detection

Probes for IncRNA MSTRG-

22387.1 (for Northern Blot)

Custom LNA ${ }^{\mathrm{TM}}$ Detection

Probes for IncRNA MSTRG-

30740.1 (for Northern Blot)

Custom LNA ${ }^{\mathrm{TM}}$ Detection

Probes for IncRNA MSTRG-

30928.1 (for Northern Blot)

Custom LNA ${ }^{\mathrm{TM}}$ Detection

Control Probes (for GAPDH)

QIAGEN

QIAGEN

QIAGEN
Cat\# 339511 LG00239367-

DDA

Cat\# 339511 LG00239368-

DDA
Cat\# 339500 LCD0168372-

BKJ

Cat\# 339511 LG00239369-

DDA

Cat\# 339511 LG00239418-

DDA

Cat\# 339511 LG00239419-

DDA

Cat\# 339500 LCD0168369-

BKJ

Cat\# 339500 LCD0168366-

BKJ

Cat\# 339508 LCD0000001-

BDJ

Custom IncRNA FISH Probe 1 Ribo Biotechnology N/A

for MSTRG-30740-1

Custom IncRNA FISH Probe 2 Ribo Biotechnology N/A

for MSTRG-30740-1

Custom IncRNA FISH Probe Ribo Biotechnology N/A

Mix for MSTRG-22387-1

Custom IncRNA FISH Probe Ribo Biotechnology N/A

Mix for MSTRG-30740-1

Custom IncRNA FISH Probe

Ribo Biotechnology

$\mathrm{N} / \mathrm{A}$

Mix for MSTRG-30928-1 

DAPT (GSIIX, LY-374973) Selleck
Cat\# S2215
Differentiating solution
Servicebio
Cat\# G1005-3
Dimethyl sulfoxide (DMSO)
Invitrogen
Cat\# D12345
DLL1 Protein, Mouse,
SinoBiological
Cat\# 50522-M08H
Recombinant (His Tag)

Ethylenediamine Tetraacetic

Sigma-Aldrich

Cat\# E5134

Acid (EDTA)

FAM labeled RNAs

Glucose

GM-CSF, recombinant, murine

Jagged 1 Protein, Human,

Recombinant (His Tag)

JetPEI reagents

Lipofectamine 2000

Lipopolysaccharide (LPS)

(from E. Coli 0111: B4)

m-18S FISH Probe Mix

(Red,20T, for mouse)

M-CSF, recombinant, human

M-CSF, recombinant, murine

Neomycin

Oligomycin A

Paraformaldehyde

Penicillin-Streptomycin

Phosphatase Inhibitor

Cocktail 2

\section{PMA}

Polyinosinic-polycytidylic acid (Poly(l:C))

Prolong ${ }^{\circledR}$ Probe - V-HTNV-S- $\quad$ ACD Bio C2

Prolong $^{\text {TM }}$ Gold Antifade Mountant

Purified NA/LE Human BD Fc Block $^{\text {TM }}$

Agilent
GenePharma

PeproTech

SinoBiological

PolyPlus

Invitrogen

InvivoGen

Ribo Biotechnology

PeproTech

PeproTech

Sigma-Aldrich

Sigma-Aldrich

Sigma-Aldrich

Sigma-Aldrich

Sigma-Aldrich

Sigma-Aldrich

InvivoGen

Thermo Fisher

BD Biosciences
Cat\# 588541-C2

Cat\# P36930

Cat\# 564765

Cat\# A07001

Cat\# 103577-100

Cat\# 315-03

Cat\# 11648-H08H

Cat\# 101-40N

Cat\# 1858793

Cat\# t|rl-3pelps

Cat\# Inc110104

Cat\# 300-25

Cat\# 315-02

Cat\# N6386

Cat\# 75351-5MG

Cat\# P6148

Cat\# P4333

Cat\# P5726

Cat\# P1585

Cat\# t|rl-pic 
Purified Rat Anti-Mouse
CD16/CD32 (Mouse BD Fc
Block $^{\mathrm{TM}}$ )

Puromycin

RBC lysis buffer (1x)

Resin

Restore $^{\mathrm{TM}}$ Western Blot

Stripping Buffer

RIPA Lysis Buffer (10x)

RNAscope ${ }^{\circledR}$ Probe $-\mathrm{Mm}$ -

MSTRG-30740-C3
Sigma-Aldrich

Gibco

Sinopharm

Thermo Fisher

Sigma-Aldrich

ACD Bio

Sigma-Aldrich

Agilent

BD Biosciences

Abcam

Sigma-Aldrich

Invitrogen

Solarbio

Solarbio

Trypsin-EDTA Solut
(with phenol red)

TSA® Plus Cy3

TSA® Plus Cy5

Type IV Collagenase

Xylene

\section{Recombinant DNA}

Akoya Biosciences

Akoya Biosciences

Sigma-Aldrich

Sinopharm
Cat\# 553141

Cat\# P9620

Cat\# 21875-034

Cat\# 10004160

Cat\# 21063

Cat\# 20-188

Cat\# 588571

Cat\# R8875-1G

Cat\# 103575-100

Cat\# 554657

Cat\# ab171523

Cat\# 93418

Cat\# 15596-018

Cat\# T1350

Cat\# T1320

Cat\# NEL744E001KT

Cat\# NEL745E001KT

Cat\# C4-BIOC

Cat\# 10023418
pcDNA3.1-S (NP)

pcDNA3.1-M (GP)

Constructed by our Lab N/A

(Wang et al., 2019)

Constructed by our Lab

$\mathrm{N} / \mathrm{A}$

(Wang et al., 2019)

pcDNA3.1-R218H
N/A
Hongyan Qin and Hua Han

Lab (Yin et al., 2009) 


\begin{tabular}{|c|c|c|}
\hline pcDNA3.1-Flag/HA-NICD & Constructed by our Lab & $\mathrm{N} / \mathrm{A}$ \\
\hline pcDNA3.1-Myc-IKKß & Constructed by our Lab & $\mathrm{N} / \mathrm{A}$ \\
\hline pcDNA3.1-Myc-IkBa & Constructed by our Lab & N/A \\
\hline pcDNA3.1-GFP/Myc-p65 & Constructed by our Lab & $\mathrm{N} / \mathrm{A}$ \\
\hline pcDNA3.1-RFP-IKBa & Constructed by our Lab & $\mathrm{N} / \mathrm{A}$ \\
\hline $\begin{array}{l}\text { pcDNA3.1- } \\
\text { Stat1/IRF5/Stat3/IRF4 }\end{array}$ & Constructed by our Lab & N/A \\
\hline pcDNA3.1- MSTRG.22387.1 & Constructed by our Lab & $\mathrm{N} / \mathrm{A}$ \\
\hline pcDNA3.1- MSTRG.30740.1 & Constructed by our Lab & N/A \\
\hline pcDNA3.1- MSTRG.30928.1 & Constructed by our Lab & $\mathrm{N} / \mathrm{A}$ \\
\hline pNF-kB-luc & Beyotime & 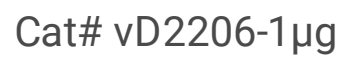 \\
\hline $\begin{array}{l}\text { PsPAX2 and pMD2.G } \\
\text { (Lentivirus System) }\end{array}$ & Conserved in our Lab & N/A \\
\hline $\begin{array}{l}\text { pLVX-Lnc-ip65-ZsGreen1 } \\
\text { (Lenti-Inc-ip65) }\end{array}$ & Constructed by our Lab & $\mathrm{N} / \mathrm{A}$ \\
\hline \multirow[t]{2}{*}{ pFastBac ${ }^{\text {TM }}$ Dual-S } & Constructed by our Lab & $\mathrm{N} / \mathrm{A}$ \\
\hline & (Cheng et al., 2016) & \\
\hline Renilla Plasmid (pRL-TK) & Conserved in our lab & $\mathrm{N} / \mathrm{A}$ \\
\hline $\begin{array}{l}\text { Truncated NICD/ p65/ IKK } \\
\text { Plasmids }\end{array}$ & Constructed by our Lab & $\mathrm{N} / \mathrm{A}$ \\
\hline Truncated Lnc-ip65 Plasmids & Constructed by our Lab & $\mathrm{N} / \mathrm{A}$ \\
\hline \multicolumn{3}{|c|}{ Experimental Models: Cell Lines \& Transgenic Mice } \\
\hline \multicolumn{3}{|l|}{ Cell Lines } \\
\hline bEnd.3 & Procell & Cat\# CL-0598 \\
\hline HEK293 & Procell & Cat\# CL-0001 \\
\hline HUVEC & Procell & Cat\# CL-0122 \\
\hline MH-S & Procell & Cat\# CL-0597 \\
\hline $\mathrm{NIH} / 3 \mathrm{~T} 3$ & Procell & Cat\# CL-0171 \\
\hline RAW264.7 & Procell & Cat\# CL-0190 \\
\hline THP-1 & Procell & Cat\# CL-0233 \\
\hline Vero E6 & ATCC & Cat\# CRL-1586 \\
\hline
\end{tabular}




\section{Transgenic Mice}

C57BL/6J

IFNAR1 Deficient (IFNAR1 ${ }^{-/}$)

C57BL/6J Mice

Lnc-ip65 Deficient (Inc-ip65 ${ }^{-/}$)

C57BL/6J Mice

NICD ${ }^{\text {STOP-floxed }}$ (Lyz2-Cre ${ }^{+}$)

C57BL/6J Mice
The Jackson Laboratory

RBP-JCKO (Lyz2-Cre+ RBP$\mathrm{J}^{\text {floxed }}$ ) C57BL/6J Mice

RIG-I Deficient (RIG-

$\mathrm{I}^{-/}$) C57BL/6J Mice

\section{Oligonucleotides}

PCR Primer Sequences, See

Table S3

RNAi Sequences, See Table

S4

\section{Software and Algorithms}

Adobe Illustrator CC 2018

Adobe Photoshop CC 2018

Bio-Plex Manager software, Version 6.0

CaseViewer

FlowJo v10

GraphPad Prism 8

Hitachi TEM system

ImageJ v1.50

Image Studio ${ }^{\text {TM }}$ Lite Software Odyssey

LightCycler ${ }^{\circledR} 96$ Application Roche

Software

R v.3.6.2
Adobe

Adobe

Bio-Rad

3DHISTECH

TreeStar

GraphPad Software

Hitachi

ImageJ

R-project
The Jackson Laboratory

Constructed by our Lab

Hongyan Qin and Hua Han Lab

(Department of Genetics and

Developmental Biology,

AFMU) (Zhao et al., 2016)

JAX stock 000664

JAX stock 010830

$\mathrm{N} / \mathrm{A}$

N/A

N/A

N/A

AFMU

N/A

N/A https://www.adobe.com/

https://www.adobe.com/

https://www.bio-rad.com/

https://www.3dhistech.com/

https://www.flowjo.com/

https://www.graphpad.com/

https://www.hitachi-

hightech.com/us/

https://www.imagej.nih.gov/ij/

https://licor.com/bio/imagestudio-lite/

https://www.roche-appliedscience.com

https://www.r-project.org/ 
Figures
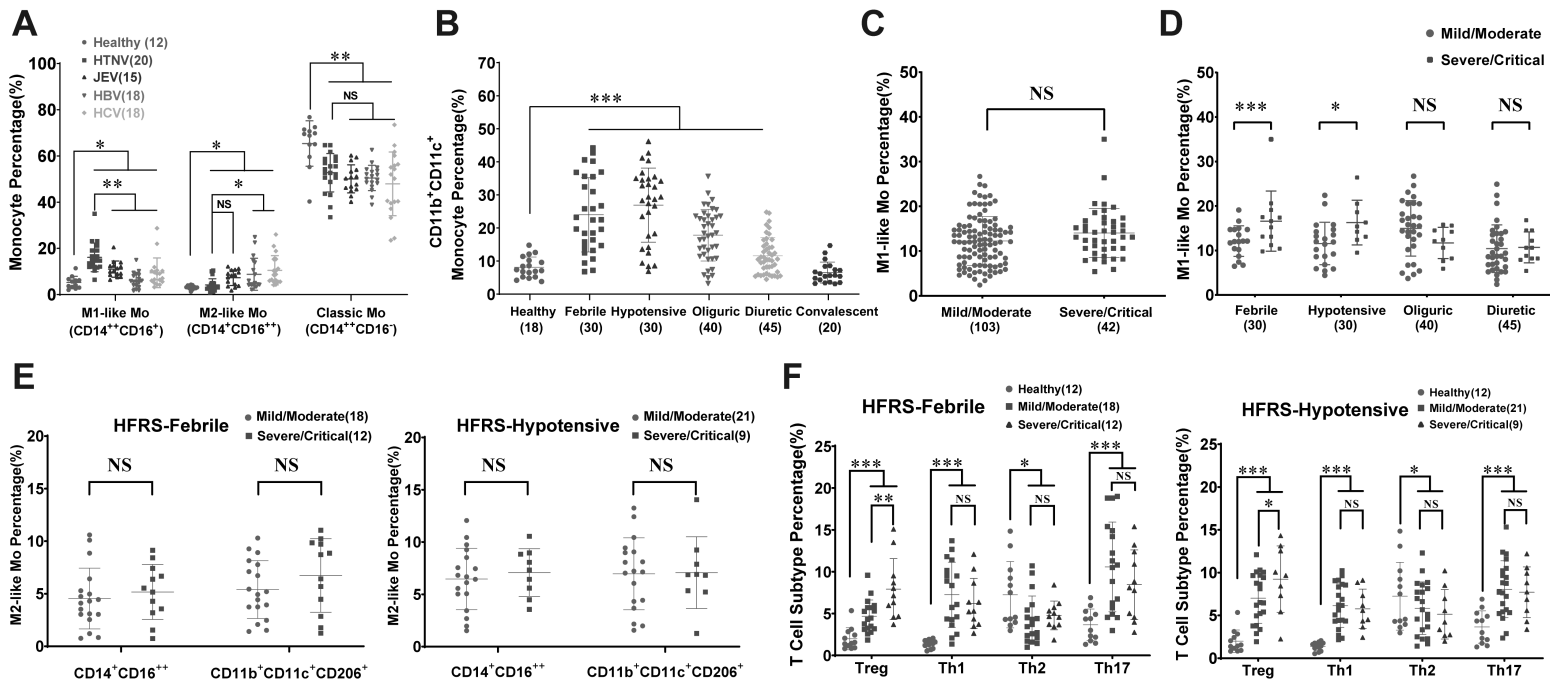

G
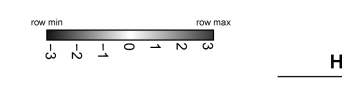

HFRS- Mild/Moderate $(39)$
Hypotensive

HFRS- Severe/Critical (21) Healthy (18)

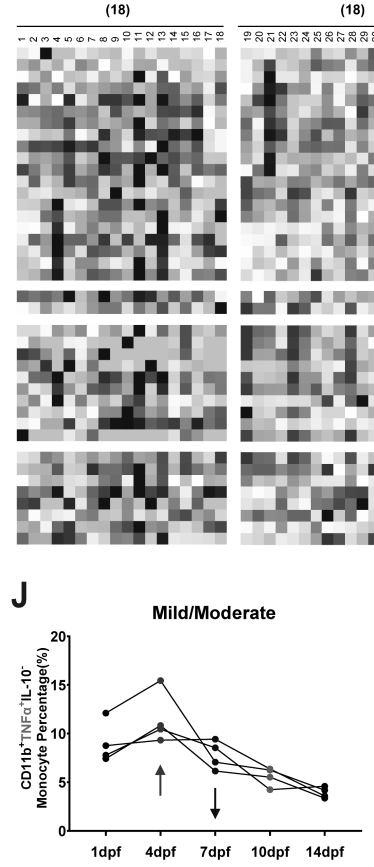

ง

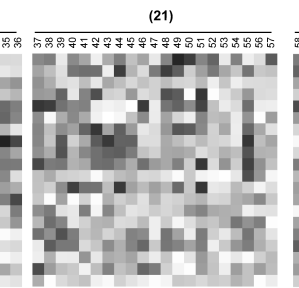

Febrile Hypotensive

(12) $\frac{\text { (9) }}{25}$

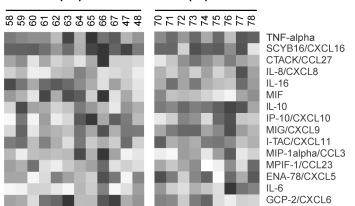

H
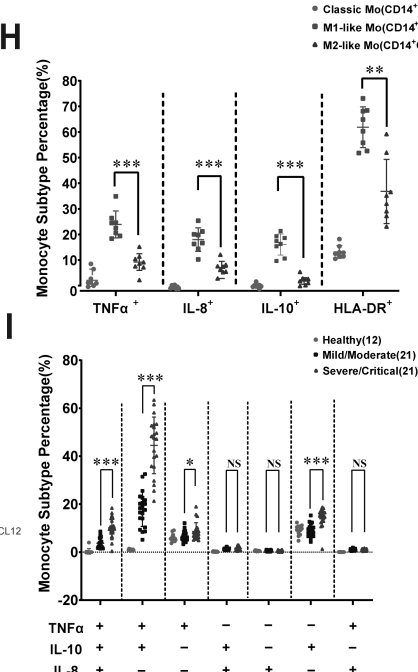

L

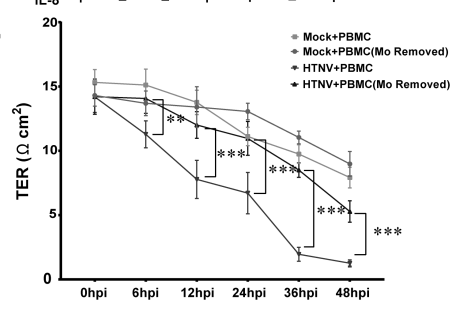

K
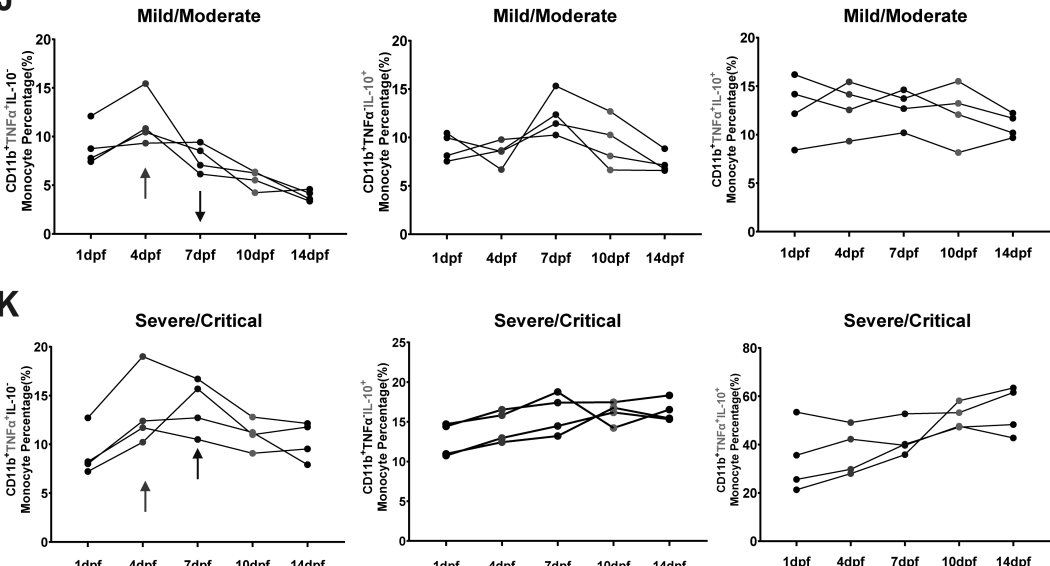

M
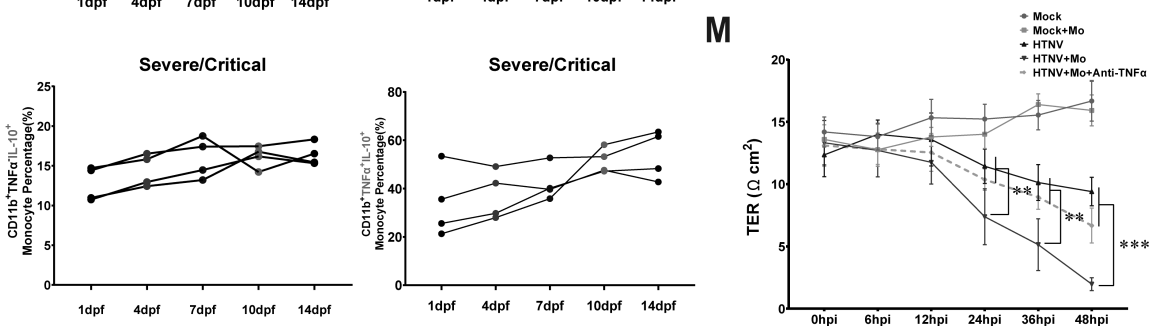

Figure 1 


\section{Inflammatory Monocyte and Macrophage Drives HFRS Progression by Triggering TNFa-centered Cytokine Storm and Aggravating Endovascular Injury in Patients}

(A) The monocyte phenotype measured by flow cytometry from PBMCs of patients with different virus infection and healthy people.

(B) The monocyte percentage in HFRS patients of five different clinical stages.

( $C$ and $D$ ) The relationship between M1-like monocyte percentage and HFRS severity, analyzed across the whole disease stage (C) or at distinct disease phases (D). To avoid the potential influence of certain medical treatments, the patients with continuous renal replacement therapy were excluded.

(E) The relationship between M2-like monocyte percentage and HFRS severity, analyzed at the febrile or hypotensive stage.

(F) T cell subsets of HFRS patients with varying disease severity at acute infection stage.

(G) Heatmap depicting the levels of 40 cytokines in the serum of patients with HFRS and healthy people.

Upregulated cytokines in HFRS patients compared with the healthy controls were marked with colorful labeling (red/green/blue), among which the upregulated (labeled with red), downregulated (labeled with green), and unchanged (labeled with blue) cytokines from patients in the severe/critical group compared with those in the mild/moderate group were labeled distinctively. Black labeling represented the cytokines that showed no discrimination in the HFRS patients compared with the healthy individuals. The heatmap of fold change was generated using after normalization with $Z$ value. $Z$ value $=(X-\mu) / \sigma$. Where, $X=$ Standardized Random Variable, $\mu$ = Sample Mean, $\sigma=$ Sample Standard Deviation.

(H) The cytokine secretion and HLA-DR expression of monocytes with different phenotypes from HFRS patients at acute stage ( $\mathrm{n}=8$ in each group).

(I) The secretion of TNFa, IL-8 and IL-10 in monocytes from HFRS patients at the acute stage.

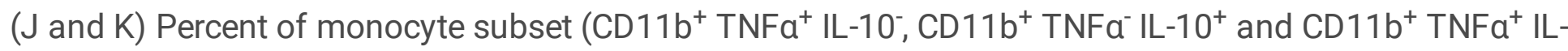
$10^{+}$) in patients with mild/moderate $(\mathrm{J})$ or severe/critical $(\mathrm{K})$ HFRS changing along with disease progression.

(L) Transendothelial electrical resistance (TER) measured through transwell experiments in a PBMCbased co-culture model with or without monocytes ( $n=8$ in each group).

(K) TER measured through transwell experiments in a monocyte-based co-culture model with or without antibodies against TNFa $(5 \mu \mathrm{g} / \mathrm{ml})(\mathrm{n}=4$ in each group).

The patient sample number in each group has been tagged on the abscissa or the figure legends $(A-1)$. Data are shown as mean $\pm S D(A-F, H$ and $I)$, or mean \pm SEM $(L$ and $M)$. Data are representative of three 
independent experiments ( $L$ and $M)$. Analysis was performed using the one-way ANOVA ( $A, B$ and $F$ ), unpaired Student's $t$ test $(\mathrm{C}-\mathrm{E}, \mathrm{H}, \mathrm{I}$ and $\mathrm{L})$, or two-way ANOVA $(\mathrm{M}) .{ }^{*} p<0.05,{ }^{\star \star} p<0.01,{ }^{\star \star \star} p<0.001$.

A (i)

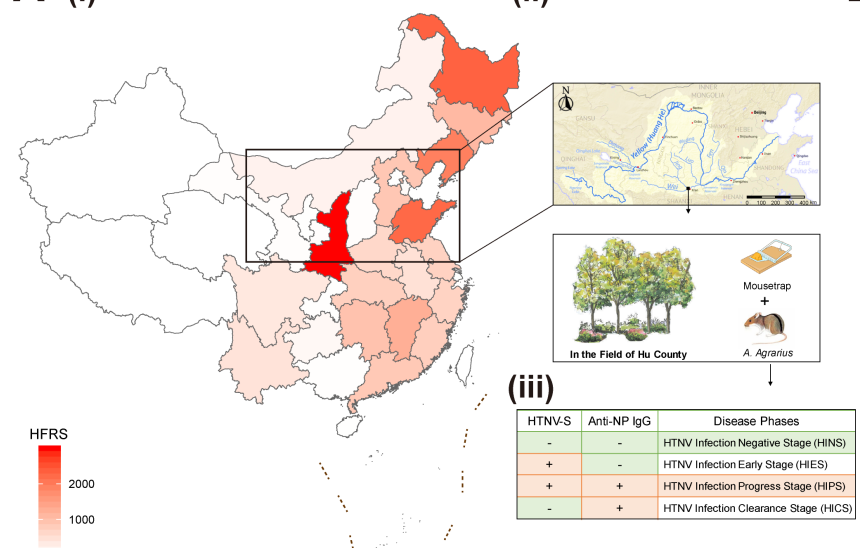

C HINS (ii) HICS
D

B
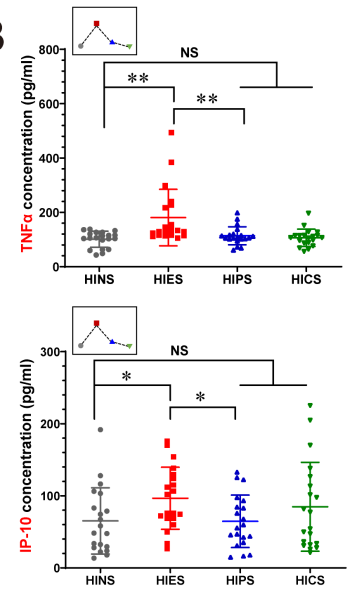
HINS MIES MIPS HICS
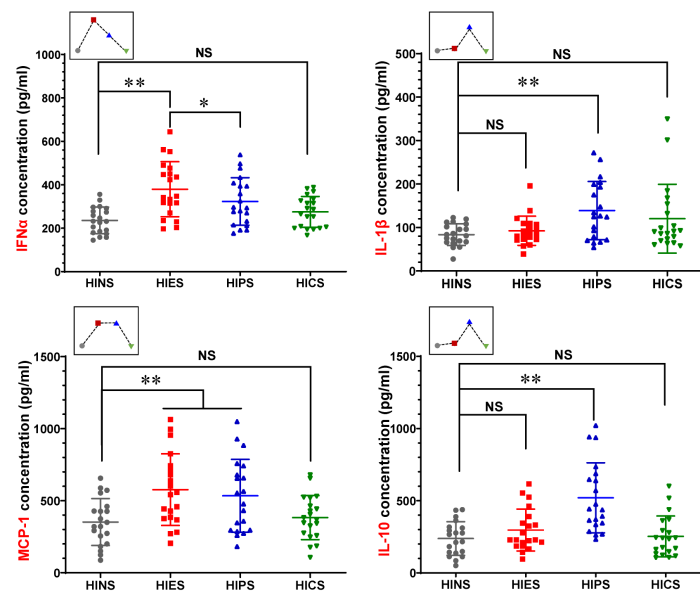

E
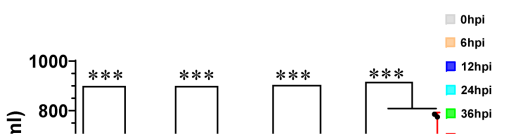

\section{Figure 2}


(A) (i) Geographic heat map depicting HFRS incidence in China from 2016 to 2017.

(ii) A. agrarius mice captured from the field of Hu County in the Weihe Plain $\left(106-110^{\circ} \mathrm{E}, 34-36^{\circ} \mathrm{N}\right)$.

(iii) Natural infection phases of HTNV in A. agrarius mice defined based on the assessment of HTNV-S and anti-NP IgG in mice lung tissue.

(B) Cytokine production measured by enzyme-linked immunosorbent assay (ELISA) in A. agrarius mice lung tissue ( $\mathrm{n}=20$ in each group). The mice samples weighing 22-28 $\mathrm{g}$ and without apparent trauma and skin infection were included for analysis. The upper left showed the variation tendency accompanied by infection progress.

(C) AMs distribution in the lung tissue detected through immunofluorescence assay. Scale bars, $200 \mu \mathrm{m}$.

(D) Immunoblot analysis of the p65 and Stat1 activation in mice AMs (every lane represented the mixture of AMs from five field mice).

(E) Supernatant cytokine concentration assessed by ELISA from primary monocytes or macrophages at different time points post HTNV infection with an MOI of 1 ( $n=4$ in each group).

(F) Immunoblot analysis of the p65 and Stat1 activation in mBMDM and hMDM from 0 hpi to 36 hpi with an $\mathrm{MOI}$ of 1 .

( $G$ and $H$ ) Immunoblot analysis of transcription factors locating in the cytoplasm or nucleus from mBMDM $(\mathrm{G})$ or hMDM $(\mathrm{H})$ at different time points post HTNV infection with an MOI of 1.

(I) The DNA binding capacity of p65 in mBMDM and hMDM from 0 hpi to 36 hpi with an MOI of 1.

(J) Supernatant TNFa production measured by ELISA from macrophage cell lines at different time points post HTNV infection with an MOI of 1.

Data are shown as mean $\pm S D(B)$, or mean $\pm S E M(E, I$ and $J)$. Data are representative of three independent experiments $(\mathrm{E}-\mathrm{J})$. Analysis of different groups was performed with the one-way ANOVA (B, $\mathrm{E}, \mathrm{I}$ and $\mathrm{J}) .{ }^{\star} p<0.05,{ }^{*} p<0.01, * \star * p<0.001$. 
A

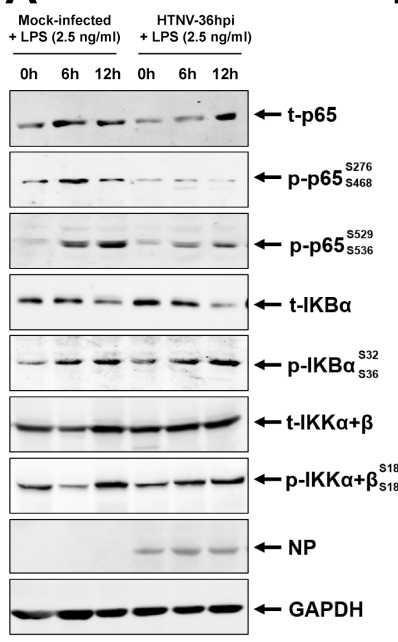

B
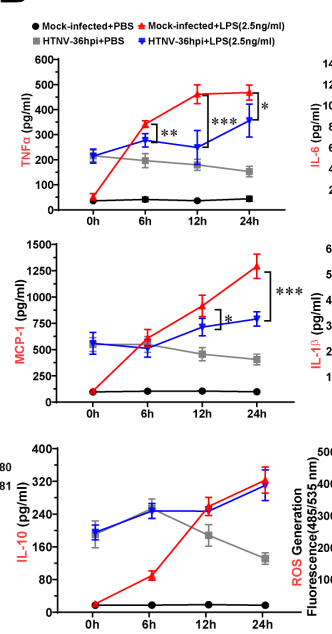

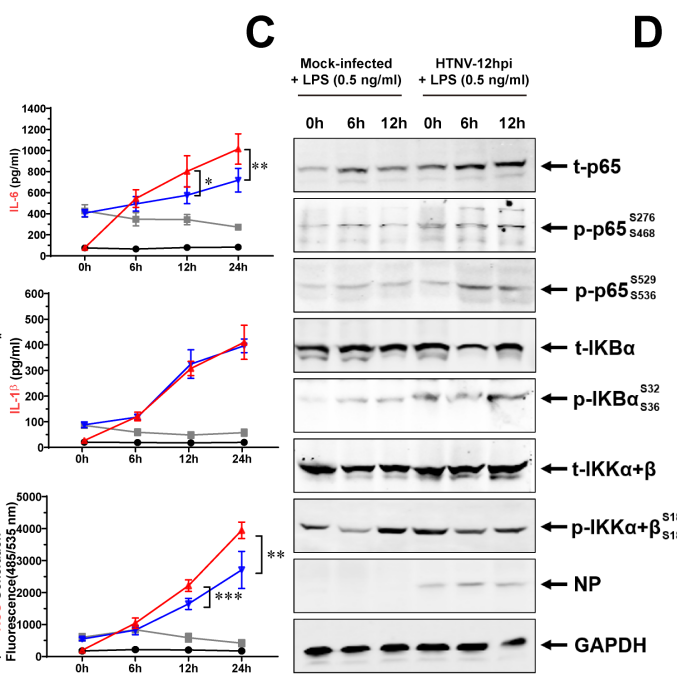

D
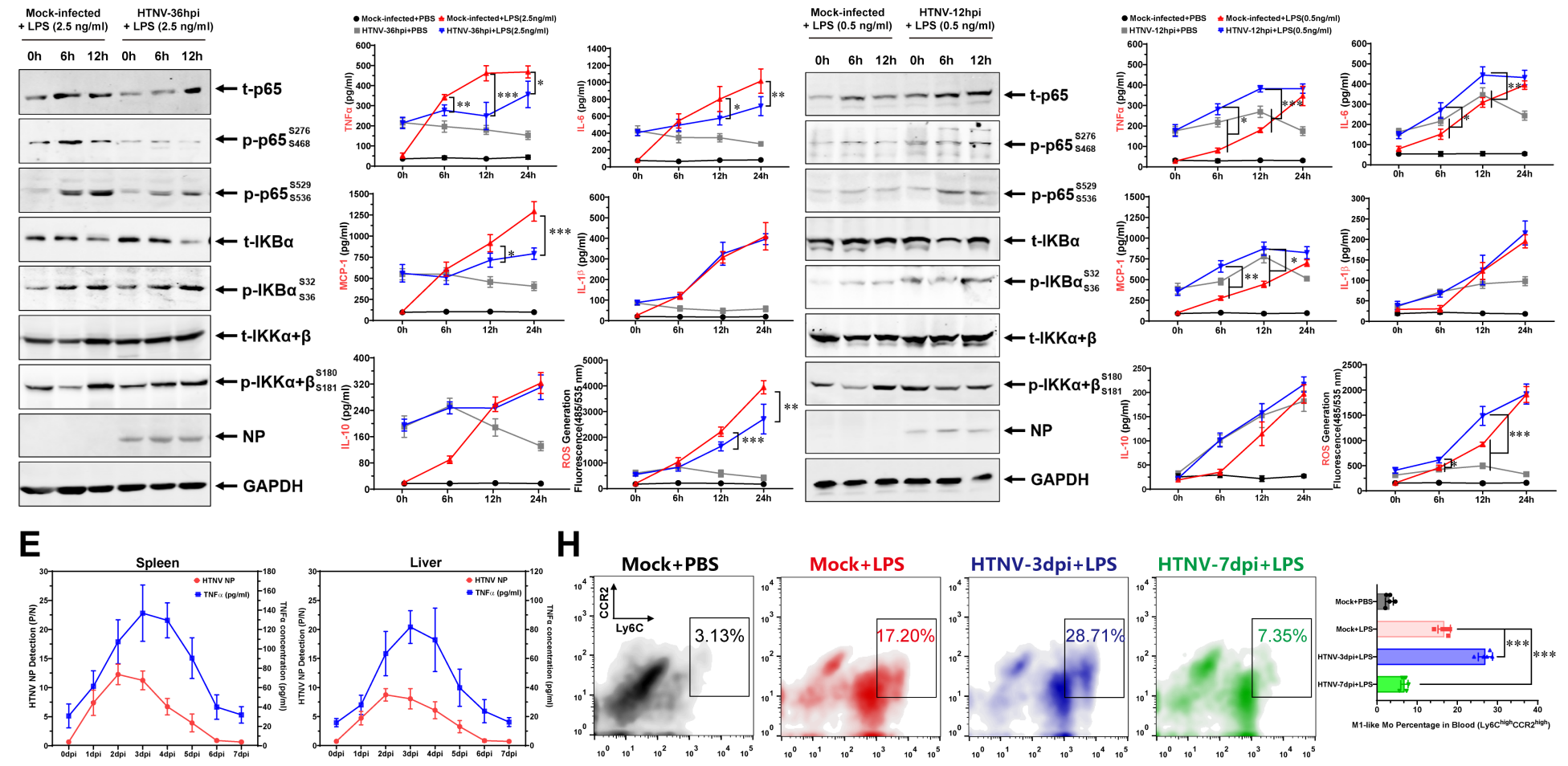

$\mathbf{H}$
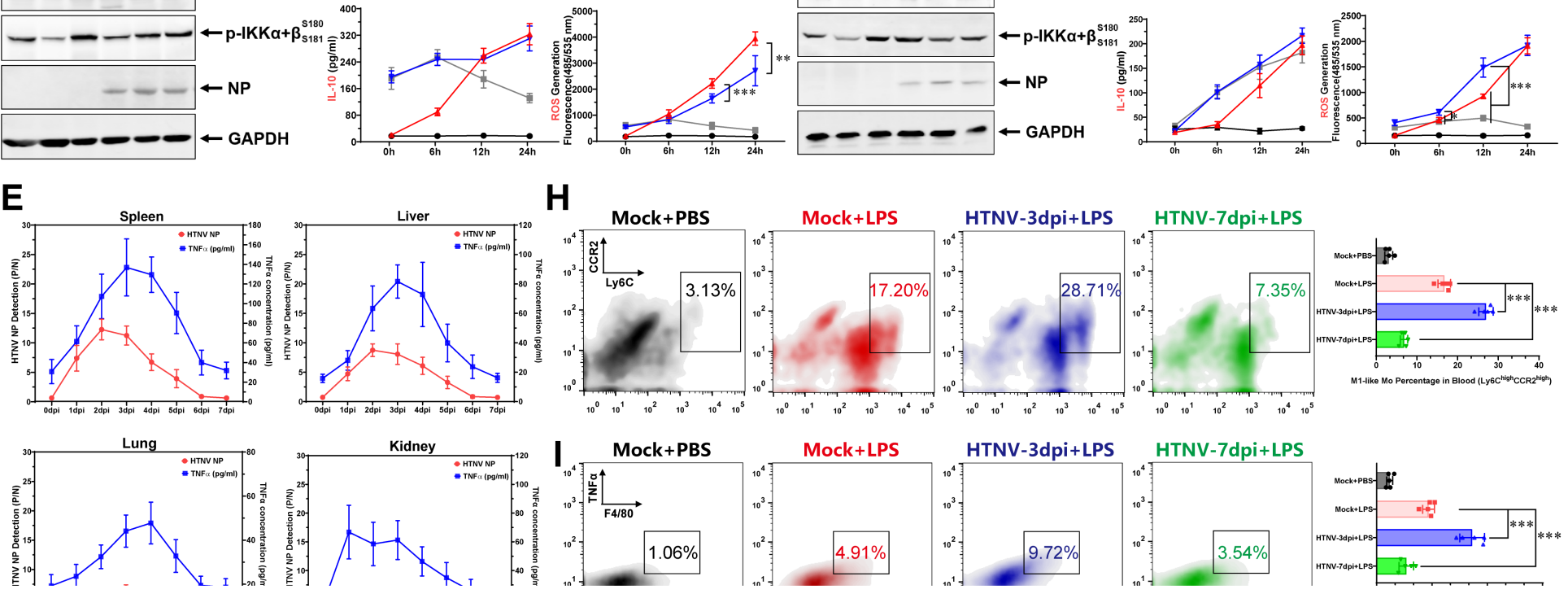

\section{Figure 3}

\section{Late-phase Inactivation of M1-type Macrophage by HTNV Alleviates Mice Inflammatory Injuries during the Secondary Bacterial Sepsis}

(A and B) Immunoblot analysis evaluating NF-KB pathway (A) and ELISA results measuring the cytokine production from supernatants, as well as the H2DCFDA-stained fluorescence values measuring cellular ROS (B). The mBMDM was mock-infected or infected by HTNV at an MOI of 1 and then stimulated by LPS $(2.5 \mathrm{ng} / \mathrm{ml})$ at $36 \mathrm{hpi}$. Samples were collected at different time points post LPS stimulation for immunoblot assay (A) or ELISA (B). 
(C and D) Immunoblot analysis evaluating NF-KB pathway (C) and ELISA results measuring the cytokine production from supernatants (D). The mBMDM was mock infected or infected by HTNV at an MOI of 1 and then stimulated by LPS $(0.5 \mathrm{ng} / \mathrm{ml})$ at $12 \mathrm{hpi}$. Samples were collected at different time points post LPS stimulation for immunoblot assay (C) or ELISA (D).

(E) HTNV-NP expression (left ordinate, red line) and TNFa concentration (right ordinate, blue line) assessed by ELISA in various tissues of HTNV infected mice (intramuscular injection, $8 \times 10^{5} \mathrm{TCID}_{50} / \mathrm{g}$ of body weight) from $0 \mathrm{dpi}$ to $7 \mathrm{dpi}$ ( $\mathrm{n}=5$ for each time point).

(F) Survival (left) and weight loss (right) data of mice with initial HTNV infection (intramuscular injection/i.m., $8 \times 10^{5} \mathrm{TCID}_{50} / \mathrm{g}$ of body weight) and succeeding LPS challenge (at 3dpi or 7dpi; intraperitoneal injection/i.p., LPS dose, $5 \mathrm{mg} / \mathrm{kg}$ of body weight).

(G) Serum TNFa and IL-6 concentration measured by ELISA from (F) at one day post LPS challenge.

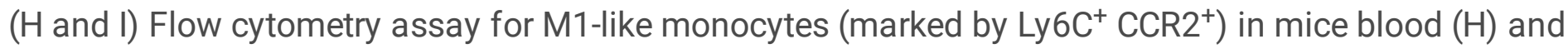
$\mathrm{M} 1 \mathrm{AMs}$ (marked by $\mathrm{F} 4 / 80^{+} \mathrm{TNFa}^{+}$) in mice lung (I) from (F). Statistical analysis was shown on the right ( $n=5$ in each group).

(J) The hematoxylin and eosin (HE) staining of mice tissues from (F) at one day post LPS challenge. Arrows, infiltrating leukocytes. Scale bars, $200 \mu \mathrm{m}$.

Data are shown as mean \pm SEM (B and $D)$, or mean \pm SD (E to I). Data are representative of two independent experiments $(\mathrm{E}-\mathrm{J})$. Analysis was performed using the unpaired Student's $t$ test (B, weight loss in $F, G$ to I), one-way ANOVA (D), or survival curve comparison (log-Rank [Mantel-Cox] test) (survival cure

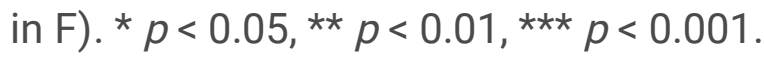




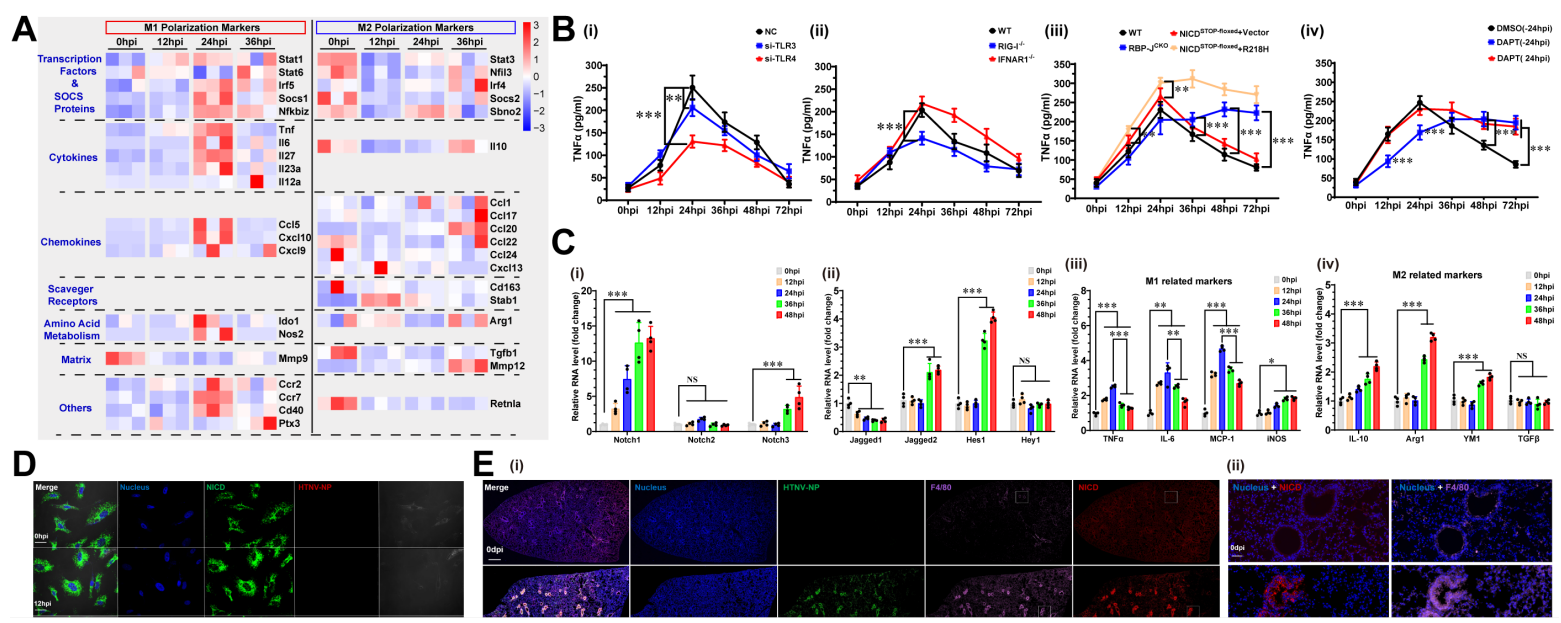

Figure 4

HTNV-induced Murine Notch Siganling Contributes to the Late-phase Inactivation of Inflammatory Macrophages

(A) Heat map showing major genes associated with macrophage polarization based on the RNA-seq analysis of $\mathrm{mBMDM}$ at different time points post HTNV infection ( $\mathrm{MOI}=1, \mathrm{n}=3$ in each group). The 
heatmap was generated using $\log _{10}$-transformed reads per kilobase of transcript per million mapped reads (RPKM) values.

(B) Supernatant TNFa concentration assessed by ELISA at different time points post infection from mBMDM that underwent distinctive treatments at an MOI of 1 ( $n=4$ in each group). (i) The mBMDM from the WT mice were transfected with negative control (NC) siRNAs or siRNAs for silencing TLR3 (si-TLR3) or TLR4 (si-TLR4) for $24 \mathrm{hr}$, and then infected with HTNV. (ii) The mBMDM generated from the WT, RIG ${ }^{-1}$ or IFNAR1\%- mice were challenged with HTNV. (iii) The mBMDM generated from the WT, RBP-JCKO (Lyz2Cre $\times$ RBP-Jfloxed, conditionally inhibiting the Notch signaling in monocytes and macrophages by knocking out RBP-J) or NICD ${ }^{\text {STOP-floxed }}$ (Lyz2-Cre $\times$ NICD $^{\text {STOP-floxed }}$, conditionally activating the Notch pathway in monocytes and macrophages by overexpressing NICD) were infected HTNV, of the which the NICD ${ }^{\text {STOP-floxed }} \mathrm{mBMDM}$ were electrotransfected with the control or vector encoding R218H. (iv) The mBMDM from the WT mice were disposed with DMSO or DAPT $(50 \mu \mathrm{mol} / \mathrm{L})$ for $24 \mathrm{hr}$ and then infected with HTNV. The DMSO or DAPT persistently existed across the whole infection process. To interpret the role of Notch pathway at the late infection phase, another group of mBMDM were treated with DMSO for $24 \mathrm{hr}$ before HTNV challenge, and then exposed to DAPT at $24 \mathrm{hpi}$.

(C) The mRNA measurement of WT mBMDM from 0 hpi to 48 hpi by qRT-PCR for murine Notch receptors (i), ligands and target genes (ii), as well as the M1 (iii) or M2 (iv) related genes, normalized to GAPDH (MOI=1, $n=4$ in each group).

(D and E) Representative immunofluorescence assays to observe the expression and subcellular localization of NICD from the WT mBMDM (D, MOI=1) or the WT lung tissue $\left(E, 8 \times 10^{5} \mathrm{TCID}_{50} / \mathrm{g}\right.$ of body weight) at different time points post HTNV infection. Scale bars, $10 \mu \mathrm{m}$ in D, $500 \mu \mathrm{m}$ in E-i and $20 \mu \mathrm{m}$ in E-ii.

(F) Heat map of macrophage polarization associated genes with the RNA-seq analysis of the WT and RBP-JCKO $\mathrm{mBMDM}$ at $36 \mathrm{hpi}$ (MOI=1, $\mathrm{n}=3$ in each group).

(G) The mRNA measurement of WT and RBP-JCKO mBMDM at 36 hpi by qRT-PCR for the M1 (upper) or $\mathrm{M} 2$ (bottom) related genes ( $\mathrm{MOI}=1, \mathrm{n}=4$ in each group).

( $\mathrm{H}$ and I) Representative flow cytometry histograms (upper) and their statistical data (bottom) of the WT and RBP-JCKO mBMDM showing the alteration of pro-inflammatory cytokine secretion $(\mathrm{H})$ or $\mathrm{CD} 80 / 86$ expression (I) (36 hpi, MOI=1, $\mathrm{n}=4$ in each group).

(J) The phagocytosis capacity of WT and RBP-JCKO mBMDM evaluated by calculating FAM ${ }^{+}$percent $(n=4$ in each group). The FAM labeled RNAs (22 bp) were added to WT or RBP-JCKO mBMDM which were infected with HTNV at an MOI of 1 for $36 \mathrm{hr}\left(3 \mu \mathrm{g}\right.$ RNAs/ $2.5 \times 10^{5}$ cells), and $24 \mathrm{hr}$ later the $\mathrm{FAM}^{+}$ macrophages were counted. Scale bars, $100 \mu \mathrm{m}$. 
(K) The chemotaxis ability of WT and RBP-JCKO mBMDM evaluated by calculating the infiltrated cells with transwell experiments ( $n=4$ in each group). Cell co-culture system was established according to the transwell experiments (membrane pore size, $5 \mu \mathrm{m}$ ) as shown in Figure S2A, whose middle and bottom layer was seeded with mBMDM and bEnd. 3 cells (murine microvascular endotheliocytes), and then HTNV was added to the system. The number of infiltrated macrophages on the back of middle layer (the region towards the bottom) was counted through crystal violet staining at $24 \mathrm{hpi}$ to evaluate the migrating capacity. Scale bars, $100 \mu \mathrm{m}$.

(L) The iNOS mRNA expression detected by qRT-PCR (left) and cellular ROS production examined by H2DCFDA-staining (right) ( $n=4$ in each group).

(M) The ECAR (i) and OCR (ii) measurement by seahorse experiments for the WT and RBP-JCKO mBMDM at 0 hpi or $36 \mathrm{hpi}$ (MOI=1, $\mathrm{n}=4$ in each group).

(N) The mitochondria morphology of WT and RBP-J ${ }^{C K O}$ mBMDM evaluated with transmission electron microscope (TEM). Left, TEM imaging; Right, calculation for the mitochondrial number and related damage percentage of each cell ( 25 cells were computed for each group).

(0) Live cell imaging depicting the translocation of GFP-p65 in cytoplasm and nucleus at late HTNV infection phase. The WT and RBP-JCKO $\mathrm{mBMDM}$ were electrotransfected with plasmids expressing GFPp65 and RFP-IKBa, and then challenged with HTNV at a MOI of 1. The Live cell imaging was recorded from $24 \mathrm{hpi}$ to $36 \mathrm{hpi}$ and four selected views of each group were shown. The corresponding data were also shown in supplementary videos (Video 3-6 for WT and Video 7-10 for RBP-JCKO mBMDM). Scale bars, $10 \mu \mathrm{m}$.

(P) Immunoblot analysis evaluating the activation of M1-related transcription factor in the WT and RBP$\mathrm{J}^{\mathrm{CKO}} \mathrm{mBMDM}$ at the late HTNV infection stage (MOI=1).

(Q) HE (Scale bars, $500 \mu \mathrm{m}$ ), TUNEL (Scale bars, $500 \mu \mathrm{m}$ ) and immunofluorescent (Scale bars, $20 \mu \mathrm{m}$ ) staining for the spleen tissues from the WT and RBP-JCKO adult mice at $7 \mathrm{dpi}\left(8 \times 10^{5} \mathrm{TCID}_{50} / \mathrm{g}\right.$ of body weight). Triangle marked the cytoplasmic location of p65 in $\mathrm{F} 4 / 80^{+}$macrophages (upper), and arrows showed the nucleus location of p65 in $\mathrm{F} 4 / 80^{+}$macrophages (bottom).

(R) Immunoblot analysis evaluating the activation of p65, JNK, ERK and IRF5 of the RBP-JCKO and WT spleens at $3 \mathrm{dpi}$ and $7 \mathrm{dpi}\left(8 \times 10^{5} \mathrm{TCID}_{50} / \mathrm{g}\right.$ of body weight).

Data are shown as mean \pm SEM, and are representative of three independent experiments. Analysis was performed using the unpaired Student's $t$ test (B-i to B-iii, and G to N), or one-way ANOVA (B-iv and C). ${ }^{*} p$ $<0.05, * \star p<0.01, * \star \star \quad p<0.001$. 


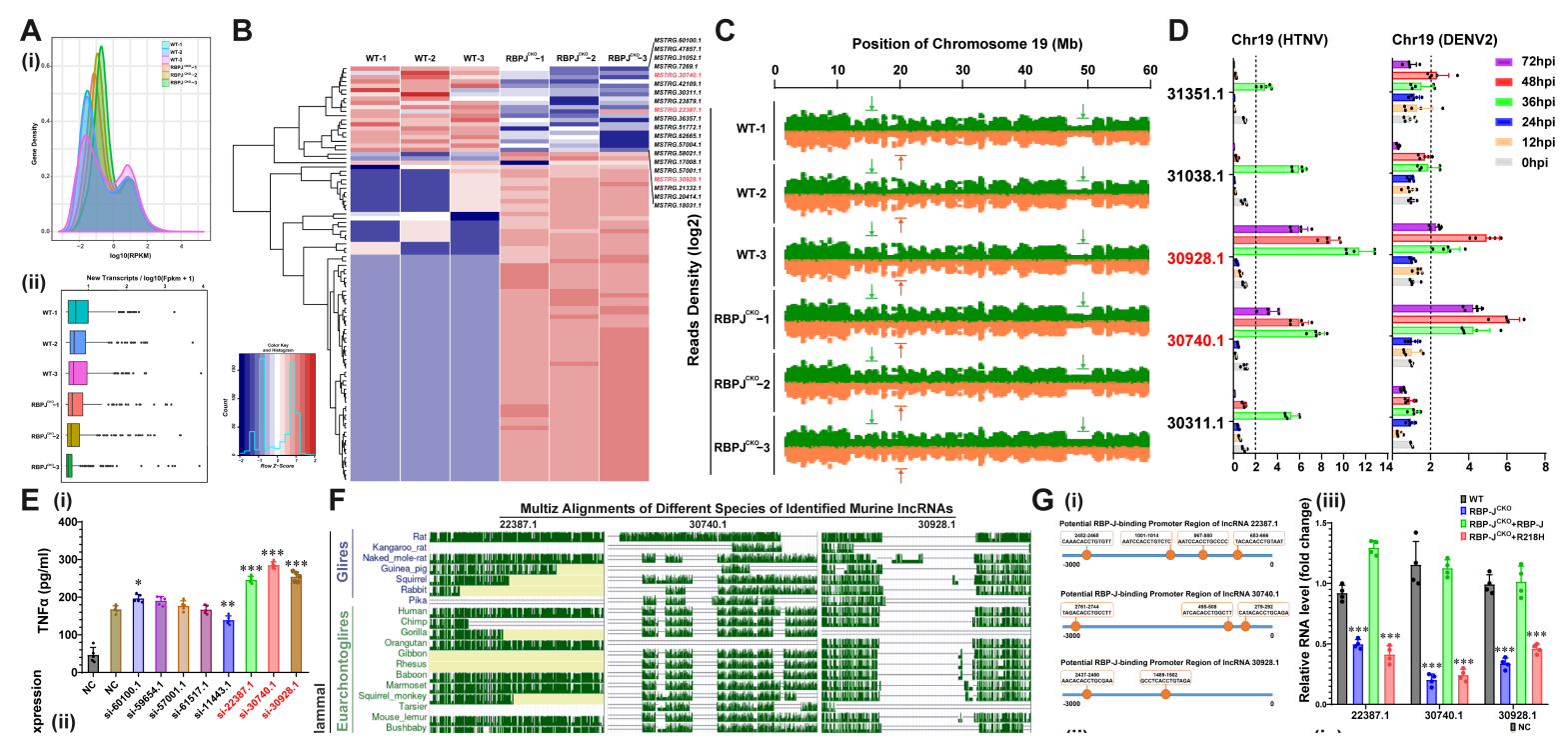

Figure 5

Murine Notch Prevents M1 Hyperactivation by Inducing Inhibitory LncRNAs

(A) The gene density of total transcripts (i) and the expression of new transcripts (ii) were shown according to the RNA-seq results of mBMDM at $36 \mathrm{hpi}$ (WT versus RBP-JCKO, MOI=1, n=3 in each group). 
(B) Heat map of novel murine-specific IncRNAs that were differentially expressed in the RBP-JCKO compared with the WT mBMDM at $36 \mathrm{hpi} \mathrm{(} \mathrm{n}=3$ in each group).

(C) The chromosomal distribution (Chr19) of differentially expressed IncRNAs from (B) as pointed by arrows ( $\mathrm{n}=3$ in each group).

(D) qRT-PCR analysis for indicated IncRNAs in HTNV or DENV2 infected mBMDM from 0 hpi to $72 \mathrm{hpi}$ (MOI=1, $n=4$ in each group).

(E) ELISA analysis for TNFa secretion from mBMDM (i) (RNAi efficiency, see Figure S8B) and luciferase detection for NF-KB activity in RAW264.7 cells (ii) (RNAi efficiency, see Figure S8C) at $36 \mathrm{hpi}(\mathrm{MOI}=1)$. The RNA interference (RNAi) experiments were performed with siRNAs at $24 \mathrm{hr}$ before HTNV infection as described in Figure 4B-i. The NC group at $36 \mathrm{hpi}$ was set as the control for statistical analysis.

(F) Sequence alignment for indicated IncRNAs of species based on the UCSC Genome Browser database.

(G) The promoter prediction of indicated IncRNAs (i). qRT-PCR analysis of them in mBMDM that were pretreated with DAPT ( $50 \mu \mathrm{mol} / \mathrm{L})$ or $\mathrm{mDIl1}(10 \mathrm{ng} / \mathrm{ml})$ (ii); were originated from WT or RBP-JCKO mice, with overexpression of RBP-J or R218H (iii); underwent RNAi experiments for $24 \mathrm{hr}$ and subsequent HTNV infection of MOI 1 for $36 \mathrm{hr}$ (iv) ( $\mathrm{n}=3$ in each group). The PBS (ii) or WT (iii) group was set as the control for statistical analysis.

(H) qRT-PCR analysis for indicated IncRNA in multiple tissues (normalized to the heart) ( $n=4$ in each group) and RNAfold prediction for their secondary structure.

(I) FISH analysis for indicated IncRNAs in mBMDM. Scale bars, $20 \mu \mathrm{m}$.

(J) qRT-PCR analysis for indicated IncRNAs in mBMDM at different time points post LPS $(5 \mathrm{ng} / \mathrm{ml})$ or polylC ( $1 \mu \mathrm{g} / \mathrm{ml}$ with liposome-based transfection) stimulation ( $\mathrm{n}=4$ in each group).

(K) qRT-PCR analysis for indicated IncRNAs in mBMDM at different time points post SeV (i, MOI=0.1) or HSV-2 (ii, MOI=0.1) infection ( $\mathrm{n}=4$ in each group).

(L) qRT-PCR (i) and Northern blot (ii) analysis for indicated IncRNAs in mBMDM at 36 hpi with different HTNV challenge doses ( $n=4$ in each group).

(M) Flow cytometry analysis for TNFa and IL-10 expression in NICD ${ }^{\text {STOP-floxed }}$ mBMDM that underwent RNAi experiments of indicated IncRNAs with LNAs for $48 \mathrm{hr}$ and subsequent HTNV infection at an MOI of 1. The $a, b$ and c subsets in (iii) were generated from the $36 \mathrm{hpi}$ group in (i). Statistical analysis of (i) and (iii) was shown in (ii) and (iv), respectively.

( $\mathrm{N}$ and 0 ) Flow cytometry analysis at 36 hpi of indicated markers for mBMDM in (M).

(P and Q) The chemotaxis (P) and phagocytosis (Q) ability analysis at $36 \mathrm{hpi}$ for mBMDM in (M). 
(R and S) Flow cytometry analysis of CD80 (R) and CD206 (S) at $36 \mathrm{hpi}$ for mBMDM in (M).

(T) ECAR examination at $36 \mathrm{hpi}$ for mBMDM in (M).

(U) Flow cytometry analysis for TNFa and IL-10 expression in RBP-JCKO mBMDM at 36 hpi. RBP-JCKO mBMDM were exogenously expressed indicated IncRNAs with lentiviruses for $72 \mathrm{hr}$, screened with puromycin for $48 \mathrm{hr}$, and infected with HTNV at an MOI of 1 for $36 \mathrm{hr}$.

Data are shown as mean \pm SEM (except $D$ ) or mean \pm SD (D). Data are representative of three independent experiments. Analysis was performed using the unpaired Student's $t$ test (G-i to G-iii), or oneway ANOVA (G-iv and $\mathrm{J}$ to $\mathrm{U}$ ). ${ }^{\star} p<0.05,{ }^{\star \star} p<0.01$, ${ }^{\star \star \star} p<0.001$. 
A

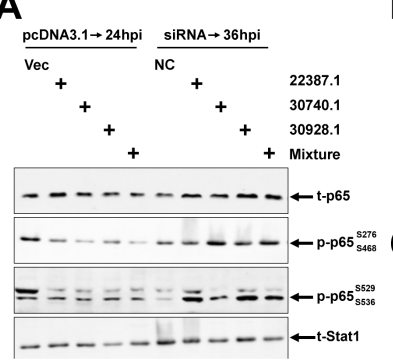

B (i)
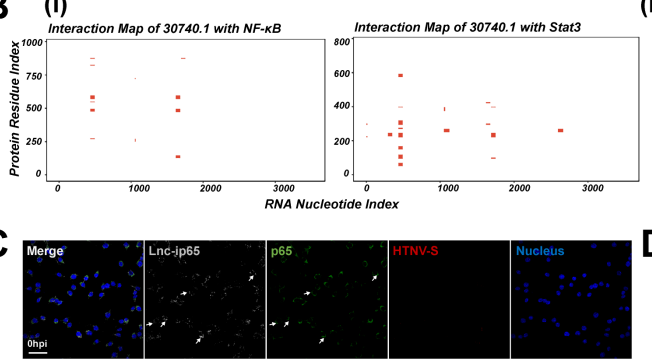
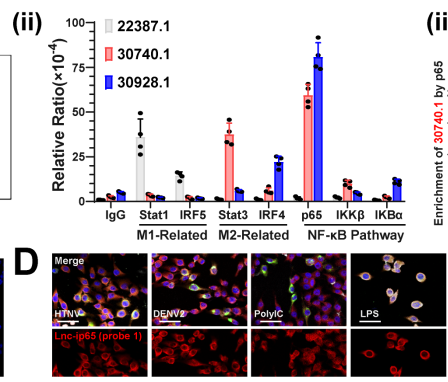

(iii)
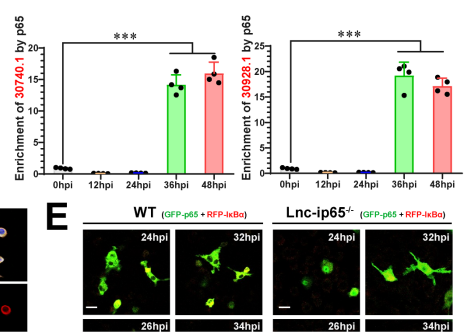

\section{Figure 6}

\section{Lnc-ip65 Binds to P65 with Its Head and Middle Structure and Hinders P65 Activation}

(A) Immunoblot analysis for mBMDM with indicated IncRNAs overexpressed at $24 \mathrm{hpi}$ or with them silenced at $36 \mathrm{hpi}$. The mBMDM were transfected with plasmids transcribing related IncRNAs or siRNAs interfering IncRNAs, and underwent HTNV infection at an MOI of $1 \mathrm{for} 24 \mathrm{hr}$ or $36 \mathrm{hr}$, respectively.

(B) Potential interaction relationship of IncRNA 30740.1 with key transcription factors predicted by with the catRAPID omics (i), as well as the RIP experiments in mBMDM exogenously expressing indicated proteins and IncRNAs (ii), or in HTNV-infected mBMDM from 0 hpi to 48 hpi (iii) ( $n=4$ in each group). 
(C) RNAScope detection for Inc-ip65 (IncRNA 30740.1, white) and HTNV S segment (viral RNA, red) in BMDM, accompanied by immunofluorescence measurement for endogenous p65 (green). Scale bars, 100 $\mu \mathrm{m}$.

(D) FISH detecting Inc-ip65 with different probes (probe 1 targeted to the 1-1000 nt, and probe 2 targeted to 1001-2000 nt) and GFP-65 in RAW264.7 cells under divergent pathological circumstances. The RAW264.7 cells stably expressing Inc-ip65 were constructed with the lentivirus system and then transfected vectors encoding GFP-p65 for $24 \mathrm{hr}$, after which these cells were in infected with HTNV or DENV at an MOI of 1 for $36 \mathrm{~h}$, or stimulated with polylC $(1 \mu \mathrm{g} / \mathrm{ml}$ with liposome-based transfection) or LPS $(5 \mathrm{ng} / \mathrm{ml})$ for $12 \mathrm{hr}$. Scale bars, $50 \mu \mathrm{m}$.

(E) Live cell imaging depicting the translocation of GFP-p65 in cytoplasm and nucleus at late HTNV infection phase. The WT and Inc-ip65 deficient mBMDM were electrotransfected with plasmids expressing GFP-p65 and RFP-IKBa, and then challenged with HTNV at a $\mathrm{MOI}$ of 1 . The Live cell imaging was recorded from $24 \mathrm{hpi}$ to $36 \mathrm{hpi}$ and four selected views of each group were shown. The corresponding data were also shown in supplementary videos (Video 11 for WT and Video 12 for Incip $65^{-/}$mBMDM). Scale bars, $10 \mu \mathrm{m}$.

(F) Immunoblot analysis for the phosphorylation of indicated proteins in WT and Inc-ip65 $5^{-1-}$ mBMDM at the late HTNV infection phase ( $\mathrm{MOI}=5)$.

(G) The RNA-binding domain prediction and related mutant construction of p65 (i). RIP analysis for the interaction of truncated p65 with Inc-ip65 in RAW264.7 cells that were overexpressed with p65 mutants and Inc-ip65 (ii).

(H) Immunofluorescent analysis in RAW264.7 cells that were overexpressed with p65 mutants and Incip65. The colocalization of truncated p65 and Inc-ip65 was shown with overexposure.

(I) Immunoblot analysis for the phosphorylation of p65 or Stat1 in RAW264.7 cells that were overexpressed with p65 (401-500 aa) and Inc-ip65 (i). Immunofluorescent analysis for the subcellular localization of exogenous and endogenous p65 in RAW264.7 cells that were overexpressed with p65 (401-500 aa) and Inc-ip65 at $0 \mathrm{hpi}$ or $36 \mathrm{hpi}$, accompanied with the FISH detecting Inc-ip65 (ii), and related statistical analysis (iii). Scale bars, $25 \mu \mathrm{m}$.

(J) Secondary structure of Inc-ip65 predicated with RNAfold and the related mutant construction. The minimum free energy (MFE) secondary structure (upper left), centroid secondary structure (upper left), and a mountain plot representation of MFE structure, the thermodynamic ensemble of RNA structures, and the centroid structure (bottom) were shown.

(K) Immunoblot analysis for the phosphorylation of p65 and IKBa in RAW264.7 cells that were exogenously expressed with different Inc-ip65 mutants at $24 \mathrm{hpi}$ with an $\mathrm{MOI}$ of 5 .

(L) Supernatant cytokine concentration detected by ELISA from (K) ( $n=4$ in each group). 
(M) Immunofluorescent analysis for the subcellular localization of myc-p65 in HTNV-infected RAW264.7 cells (MOI=5, $24 \mathrm{hpi}$ ) that were overexpressed with p65 and Inc-ip65 mutants, accompanied with the RNAScope measurement for Inc-ip65. Scale bars, $10 \mu \mathrm{m}$.

(N) RIP analysis for the interaction of truncated p65 with Inc-ip65 mutants in RAW264.7 cells ( $n=4$ in each group).

Data are shown as mean \pm SEM, and are representative of three independent experiments. Analysis was performed using the one-way ANOVA (G-iv and $\mathrm{J}$ to $\mathrm{U}$ ). ${ }^{*} p<0.05,{ }^{* \star} p<0.01,{ }^{\star \star *} p<0.001$. 
A
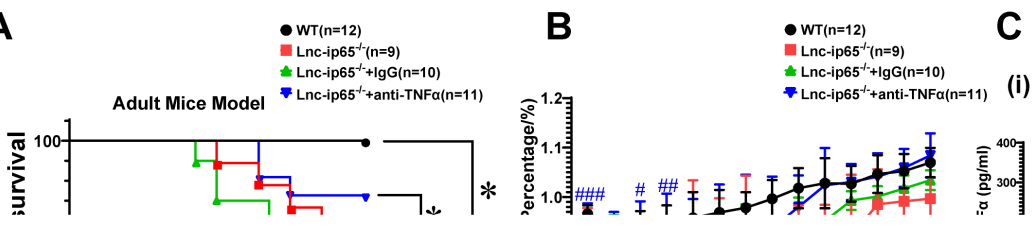

C

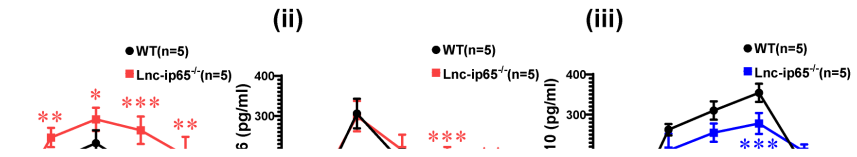

\section{Figure 7}

Exacerbated Inflammation and Tissue Injury Correlate with Disease Progression in HTNV-infected Lncip65 $5^{-/-}$Mice

(A and B) Survival (A) and weight loss (B) data for 8-week-old WT or Inc-ip65 $5^{-/-}$mice with high HTNV challenge dose (i.m., $8 \times 10^{7} \mathrm{TCID}_{50} / \mathrm{g}$ of body weight). The Inc-ip $65^{-/-}$mice were treated with IgG or TNFa neutralizing antibody $(5 \mu \mathrm{g} / \mathrm{g}$ ) every two days from $3 \mathrm{dpi}$ (shown with green or blue line). The significance 
of weight comparison was marked with blue pound signs (blue versus green line) or red asterisks (red versus black line).

(C) Serum cytokine concentration measured by ELISA from 0 dpi to 8 dpi ( $n=5$ in each group).

(D) qRT-PCR analysis for indicated genes in the lung tissues at 4 dpi or $6 \mathrm{dpi}$ ( $\mathrm{n}=4$ in each group).

(E) HE (Scale bars, $500 \mu \mathrm{m}$ for the upper and $50 \mu \mathrm{m}$ for the bottom) (i), TUNEL (Scale bars, $500 \mu \mathrm{m}$ ) (ii) and immunofluorescent staining (Scale bars, $50 \mu \mathrm{m}$ ) (iii to iv) for the lung tissues at 6 dpi. Arrows in (i) pointed to the exudative inflammation region. Arrows, asterisks and pound signs in (iii) showed the iNOS ${ }^{+}$ $\mathrm{NICD}+\mathrm{AMs}\left(\mathrm{F} 4 / 80^{+}\right)$, alveolar epithelial and stromal cells, respectively, which in (iv) indicated the p-Stat $1^{+}$ or p-p65 $5^{+}$cells. Arrows in (v) showed F4/80 $0^{+} \mathrm{NP}^{+}$cells.

(F) Immunoblot analysis for the phosphorylation of p65 and Stat1 in AMs at 0 dpi or 6 dpi (every lane represented the mixture of AMs from five WT or Inc-ip65 $5^{-/-}$mice).

(G) qRT-PCR analysis for indicated genes in the liver tissues at 4 dpi or $6 \mathrm{dpi}(\mathrm{n}=4$ in each group).

(H) HE, TUNEL and immunofluorescent staining for the liver tissues similar to (E). Arrows showed the infiltrated inflammatory cells and asterisks marked the hepatocytes with pyknosis in (i). Arrows in (iii) showed the $\mathrm{F} 4 / 80^{+} \mathrm{NOS}^{+} \mathrm{NICD}^{+}$cells. Arrows or asterisks in (iv) pointed to the $\mathrm{p}-\mathrm{p} 65^{+}$or $\mathrm{p}$-Stat $1^{+} \mathrm{AMs}$ $\left(\mathrm{F} 4 / 80^{+}\right)$, respectively. Arrows in (v) indicated the $\mathrm{NP}^{+}$cells.

(I) Immunoblot analysis for the phosphorylation of p65 and Stat1 in KCs at 0 dpi or 6 dpi (every lane represented the mixture of KCs from three WT or Inc-ip $65^{-/-}$mice).

(J) qRT-PCR analysis for indicated genes in the spleen tissues at 4 dpi or $6 \mathrm{dpi}$ ( $\mathrm{n}=4$ in each group).

(K) HE, TUNEL and immunofluorescent staining for the spleen tissues similar to (E). Arrows in (iii) showed the $\mathrm{F} 4 / 80^{+} \mathrm{NOS}^{+} \mathrm{NICD}^{+}$cells. Arrows or asterisks in (iv) pointed to the $\mathrm{p}-\mathrm{p} 65^{+}$or $\mathrm{p}$-Stat $1^{+} \mathrm{AMs}\left(\mathrm{F} 4 / 80^{+}\right)$, respectively. Arrows in (v) indicated the $\mathrm{F} 4 / 80^{+} \mathrm{NP}^{+}$cells.

(L) Representative flow cytometry analysis for macrophage polarization in the spleens (i) and related statistical analysis ( $n=4$ in each group) at $6 \mathrm{dpi}$.

(M) The inflammation score evaluation of various organs from WT and Inc-ip6 $65^{-1-}$ mice at $4 \mathrm{dpi}$ and $6 \mathrm{dpi}$ ( $n=4$ in each group).

(N) The heat (i) and mechanical (ii) hypersensitivity from WT and Inc-ip $65^{-/-}$mice from $0 \mathrm{dpi}$ and $30 \mathrm{dpi}$ ( $n=4$ in each group).

(0) Survival data for 8-week-old WT or Inc-ip $65^{-/-}$mice with LPS (i.p., $5 \mathrm{mg} / \mathrm{kg}$ of body weight) or CS (i.p. $0.6 \mathrm{mg} / \mathrm{g}$ of body weight) challenge. 
Data are shown as mean $\pm \mathrm{SD}$, and are representative of two independent experiments. Analysis was performed mainly with the unpaired Student's $t$ test, or the survival curve comparison (log-Rank [MantelCox] test) (survival cure in $\mathrm{A}$ and $\mathrm{O}$ ) or Mann Whitney $\mathrm{U}$ test $(\mathrm{N}) .{ }^{*} p<0.05,{ }^{\star \star} p<0.01,{ }^{\star \star \star} p<0.001 .{ }^{\#} p<$ $0.05,{ }^{\#} p<0.01,{ }^{\# \# \#} p<0.001$.

\section{Figure 8}

\section{NICD Generated at Early HTNV Infection Phase Accelerated P65-mediated Inflammatory Macrophage Activation}

(A) The crosstalk between Notch and NF-KB pathway based on the STRING database.

(B) Co-immunoprecipitation (co-IP) analysis for the interaction between HA-NICD and myc-tagged NF-KBinvolved proteins in NIH/3T3 cells that were exogenously expressed with indicated vectors. Anti-HA (i) or anti-Myc (ii) antibodies were applied to pull down myc-tagged p65/ IKK $/$ / IKBa or HA-NICD, respectively.

(C) Immunofluorescent observation for the colocalization of NICD with p65 (i) or IKKß (ii) post HTNV infection in $\mathrm{mBMDM}(\mathrm{MOI}=1)$.

(D) Immunoblot analysis for NF-KB pathway in RBP-JCKO mBMDM that were transfected with control vector or the vector encoding NICD for $24 \mathrm{hr}$ and then infected with HTNV at an MOI of 0.5 (i).

Immunoblot analysis for the activation of p65 and Stat1 in WT mBMDM that were treated with DAPT (50 $\mu \mathrm{mol} / \mathrm{L}$ ) from -24 hpi and infected with HTNV (MOI=5) (ii).

(E) Schematic diagram for constructing protein mutants of p65, NICD and IKK $\beta$.

(F) Co-IP analysis for the interaction between HA-NICD and truncated p65 tagged by GFP in NIH/3T3 cells that were transfected with indicated vectors. Anti-HA (i) or anti-GFP (ii) antibodies were applied to pull down GPF-tagged p65 mutants or HA-NICD, respectively.

(G) Co-IP analysis for the interaction between myc-p65 and truncated flag-tagged NICD in NIH/3T3 cells that were transfected with indicated vectors. Anti-myc (i) or anti-flag (ii) antibodies were applied to pull down flag-tagged NICD mutants or myc-p65, respectively.

(H) Co-IP analysis for the interaction between flag-NICD and truncated myc-tagged IKK $\beta$ in NIH/3T3 cells that were transfected with indicated vectors. Anti-flag (i) or anti-myc (ii) antibodies were applied to pull down myc-tagged IKK $\beta$ mutants or flag-NICD, respectively.

(I) Co-IP analysis for the interaction between myc-IKK $\beta$ and truncated flag-tagged NICD in NIH/3T3 cells that were transfected with indicated vectors. Anti-myc (i) or anti-flag (ii) antibodies were applied to pull 
down flag-tagged NICD mutants or myc-IKKß, respectively.

(J) Co-IP analysis for the interaction of HA-NICD with myc-p65 or my-IKK $\beta$ in NIH/3T3 cells that were cotransfected with vectors transcribing Inc-ip65 and its mutants.

(K) Immunoblot analysis for the phosphorylation of p65, JNK or ERK in hMDM at indicated time points post HTNV infection at an $\mathrm{MOl}$ of 5 , which were treated with hJagged $1(25 \mathrm{ng} / \mathrm{ml})$ or DAPT $(15 \mu \mathrm{mol} / \mathrm{L})$ from -24 hpi.

(L) Immunoblot analysis for the activation of M1- or M2-related transcription factors in hMDM at indicated time points post HTNV infection at an $\mathrm{MOI}$ of 5 , which were pre-infected with lentivirus for $72 \mathrm{hr}$ to exogenously expressing Inc-ip65.

(M) Supernatant cytokine concentration detected by ELISA from (L) ( $n=4$ in each group).

(N) qRT-PCR analysis for the pro- and anti-inflammatory gene expression of hMDM from ( $L$ ) ( $n=4$ in each group).

(0) The potential interaction of hantaviral proteins with host factors predicated with P-HIPSTer.

(P) Immunoblot analysis for NICD generation in mBMDM that were infected with HTNV or Heat/Co $\mathrm{CO}^{6}$ devitalized HTNV (MOI=5), or transfected with viral RNAs (S or M), or stimulated with viral proteins (NP with $1 \mu \mathrm{g} / \mathrm{ml}$ or VLP with an MOI of 5) for $12 \mathrm{hr}$ (i). qRT-PCR analysis of TNFa (ii) or Hes1 (iii) of mBMDM in (i). qRT-PCR analysis of inflammatory genes (iv) of NP-stimulated mBMDM that were treated with DAPT for $-12 \mathrm{hr}$ to $12 \mathrm{hr}$ ( $\mathrm{n}=4$ in each group).

(Q) Flow cytometry analysis for $\mathrm{TNFa}^{+}$or $\mathrm{iNOS}^{+} \mathrm{mBMDM}$ at $24 \mathrm{hr}$ post NP stimulation $(5 \mu \mathrm{g} / \mathrm{ml})$. The mBMDM were pretreated with DMSO or DAPT for $12 \mathrm{hr}$ and then underwent NP stimulation. The NP (5 $\mu \mathrm{g} / \mathrm{ml}$ ) was co-incubated with control antibody $4 \mathrm{G} 2$ (the flavivirus group antibody, $50 \mathrm{ng} / \mathrm{ml}$ ) or anti-NP antibody $1 \mathrm{~A} 8(50 \mathrm{ng} / \mathrm{ml})$ for $2 \mathrm{~h}$ at room temperature (RT), and then added to stimulate mBMDM $(\mathrm{n}=4 \mathrm{in}$ each group).

(R) Flow cytometry analysis for $\mathrm{CD} 80^{+} \mathrm{TNFa}^{+}$or $\mathrm{CD} 14^{+} \mathrm{CX} 3 \mathrm{CR} 1^{+}$mBMDM at $36 \mathrm{hr}$ post $\mathrm{HTNV}$ infection or NP mutant stimulation. Challenge dose of HTNV, MOI=1. The truncated NP peptides were constructed and purified with the baculovirus system as we previously constructed (Cheng et al., 2016) (1.3NP, full length; 0.7NP, containing 1-230 aa translated from 1-690 nt; 0.3NP, containing 1-90 aa translated from 1$270 \mathrm{nt}$ ). Stimulation dose of NP mutants, $5 \mu \mathrm{g} / \mathrm{ml}$. 0.3NP+1A8 group was stimulated with 0.3NP that had been pre-incubated with $1 \mathrm{~A} 8$ for $2 \mathrm{~h}$ at RT. 0.3NP+DAPT group was pretreated with DAPT for $12 \mathrm{hr}$.

(S) Survival data for 4-day neonatal mice with HTNV challenge (i.p. $8 \times 10^{5} \mathrm{TCID}_{50} / \mathrm{g}$ of body weight) and then treated with $4 \mathrm{G} 2$ or $1 \mathrm{~A} 8(0.25 \mu \mathrm{g} / \mathrm{g}$ of body weight) from $1 \mathrm{dpi}$ to death (every two days). 
(T) Flow cytometry analysis for the activation of MPS in neonatal mice at 3 dpi (HTNV challenging, i.p. $8 \times 10^{5} \mathrm{TCID}_{50} / \mathrm{g}$ of body weight), especially for the $\mathrm{CCR} 2^{+} \mathrm{CX} 3 \mathrm{CR} 1^{+}$cells from peripheral blood monocytes (CD11 $\mathrm{b}^{+}$Ly6C $\mathrm{C}^{+}$) (i), CD11 $\mathrm{b}^{\text {medium }}$ Ly6 $\mathrm{C}^{\text {high }}$ inflammatory monocytes/macrophages in the spleen, or iNOS ${ }^{+} \mathrm{CD}_{206^{-}} \mathrm{M}^{-}$as well as iNOS ${ }^{-} \mathrm{CD}^{2} 26^{+} \mathrm{M} 2$ polarization in the spleen.

(U) Survival data for 4-day neonatal mice with HTNV challenge (i.p. $8 \times 10^{5} \mathrm{TCID}_{50} / \mathrm{g}$ of body weight) and then treated with 3D8 $(0.05 \mu \mathrm{g} / \mathrm{g}$ of body weight) at $1 \mathrm{dpi}$ or $5 \mathrm{dpi}$, or 3D8 (0.05 $\mu \mathrm{g} / \mathrm{g}$ of body weight) combined with $4 \mathrm{G} 2(0.25 \mu \mathrm{g} / \mathrm{g}$ of body weight $)$ or $1 \mathrm{~A} 8(0.25 \mu \mathrm{g} / \mathrm{g}$ of body weight $)$ at $5 \mathrm{dpi}$ every two days till death.

Data are shown as mean \pm SEM, and are representative of two independent experiments. Analysis was performed mainly with the unpaired Student's $t$ test, or the survival curve comparison (log-Rank [MantelCox] test) (survival cure in $\mathrm{S}$ and $\mathrm{U}$ ). ${ }^{\star} p<0.05,{ }^{\star *} p<0.01,{ }^{\star \star \star} p<0.001$.

\section{Supplementary Files}

This is a list of supplementary files associated with this preprint. Click to download.

- FigureS1.tif

- Figures2.tif

- FigureS3.tif

- FigureS4.tif

- FigureS5.tif

- Figures6.tif

- FigureS7.tif

- Figures8.tif

- FigureS9.tif

- FigureS10.tif

- Figures11.tif

- Video1.mp4

- Video2.mp4

- Video3.mp4

- Video4.mp4

- Video5.mp4

- Video6.mp4

- Video7.mp4 
- Video8.mp4

- Video9.mp4

- Video10.mp4

- Video11.mp4

- Video12.mp4

- SupplementaryTables.rar

- GraphicalAbstract.tif 